\title{
Steady-state dynamic behavior of an on-board rotor under combined base motions
}

\author{
Mzaki Dakel, Sébastien Baguet and Régis Dufour \\ Université de Lyon, CNRS, INSA-Lyon, LaMCoS UMR5259, F-69621, France
}

\begin{abstract}
In the transportation domain, on-board rotors in bending are subjected not only to rotating mass unbalance but also to several movements of their base. The main objective of this article is to predict the dynamic behavior of a rotor in the presence of base excitations. The proposed on-board rotor model is based on the Timoshenko beam finite element. It takes into account the effects corresponding to the rotary inertia, the gyroscopic inertia, the shear deformation of shaft as well as the geometric asymmetry of disk and/or shaft and considers six types of deterministic motions (rotations and translations) of the rotor rigid base. The use of Lagrange's equations associated with the finite element method yields the linear second-order differential equations of vibratory motion of the rotating rotor in bending relative to the moving rigid base which forms a non-inertial frame of reference. The linear equations of motion highlight periodic parametric terms due to the geometric asymmetry of the rotor components and time-varying parametric terms due to the rotational motions of the rotor rigid base. These parametric terms are considered as sources of internal excitation and can lead to lateral dynamic instability. In the presented applications, the rotor is excited by a rotating mass unbalance combined with constant rotation and sinusoidal translation of the base. Quasi-analytical and numerical solutions for two different rotor configurations (symmetric and asymmetric) are analyzed by means of stability charts, Campbell diagrams, steady-state responses as well as orbits of the rotor.
\end{abstract}

\section{Keywords}

Rotordynamics, asymmetric rotor, on-board rotor, finite elements, base excitation, parametric excitation, transient motion, dynamic instability.

\section{Introduction}

Rotating machines play a paramount role in many modern industrial applications. Most of them can be considered as on-board machines affected mainly by mass unbalance and support excitations. Automotive turbocharger, ship and aircraft turbines as well as locomotive electrical generators are examples of rotors on moving base. The undesirable mass unbalance is due to the eccentricity of the center of mass along the rotor axis and can be caused by material non-homogeneities, manufacturing defaults, assembly and service conditions. The rotor balancing aims at minimizing the mass unbalance but generally does not lead to a complete cancellation, see, for example, Kang et al. (2003) and Levecque et al. (2011). A rotor can also be excited by the movement of its base which can increase the lateral vibration of the rotor and create a dynamic instability phenomenon. Therefore a rotor dynamic model taking into account multi-source excitations is of prime interest at the design stage in order to product rotating machines as reliable as possible and avoid catastrophic failure during the operation phase.

The literature comprises numerous books concerning the prediction of dynamics of symmetric/asymmetric rigid/flexible rotor systems mounted on linear/non-linear elastic bearings in the case of a fixed base (Rao, 1992; Lalanne and Ferraris, 1998; Genta, 2005). The asymmetry due to the disk, the shaft and/or the bearing was treated in some works. Guilhen et al. (1988) noted that differential equations without periodic coefficients could be obtained either in the inertial coordinate system or in the frame moving with the rotor. The dynamic stability was investigated using the Floquet theory and the mass unbalance response was computed with the Newmark scheme. Kang et al. (1992) employed the Timoshenko beam finite elements (FEs) for modeling rotor-bearing systems by taking into account the asymmetry of disk, shaft and/or bearing. They calculated the mass unbalance response using the harmonic balance method. According to their work, the resonant speeds could change due to various angles between major axes of disk and shaft, the shaft asymmetry as well as the bearing characteristics. Oncescu et al. (2001) generalized a FE procedure for rotor-bearing systems to include the effects of the 
asymmetry of shaft and/or bearing. They gave the matrices corresponding to the asymmetric shaft FE and applied the time-transfer matrix method based on the Floquet theory to the linear equations of motion with periodic coefficients expressed in the inertial frame of reference. As shown in their study, the indicated method was a convenient way for predicting the dynamic behavior of the asymmetric rotor-bearing systems. Sabuncu and Evran (2006) studied the motion stability of an asymmetric blade induced by a lateral parametric excitation using the Timoshenko beam FEs. They discussed the effects of the shear coefficient, the beam length variation, the rotational speed and the coupling due to the center of flexure distance from the centroid on the dynamic instability regions. Beley-Sayettat (1994) studied the influence of the shaft asymmetry and the earthquake on the dynamic behavior of a rotor. In the case of an asymmetric shaft, the equations of motion are developed in the Galilean frame (fixed in space) and in the rotating frame using the Rayleigh-Ritz method and the FE method. Employing the Floquet theory for the stability and the Newmark method for the dynamic response, the behavior of a turbogenerator of aircraft carriers was investigated in the cases of a construction asymmetry (winding) and an accidental asymmetry (crack). In the case of a seismic motion, the equations of motion were derived for the rigid support (mono-excitation) and the flexible support (multi-excitation). A spectral method with complex modes was employed for solving the equations of motion.

Many studies concentrated on the dynamic behavior of a rotor under seismic or shock excitations of its base. Hori and Kato (1990) examined a seismic response of a Jeffcott rotor supported by oil film bearings to a real seismic wave and investigated its stability by calculating the locations of the disk and journal centers (using the Runge-Kutta-Gill method). Suarez et al. (1992) presented without any derivation the equations of motion of a FE rotor model subjected to six components of base excitations and studied its seismic response. They observed that even for strong rotational inputs, the parametric terms in these equations could be neglected without affecting the rotor response. Gaganis et al. (1999) considered the non-linear bearing coefficients to be piecewise constant and modeled the excitation due to the earthquake as a movement of the base of the rotor-bearing systems using the El Centro earthquake data. In their work, the response and the variation of the dynamic properties of the rotorbearing systems were investigated. Bachelet et al. (2006) predicted the dynamic behavior of an asymmetric rotor excited by a seismic translational base excitation and developed an original spectral approach to approximate the rotor response. They discussed the results of the spectral approach through a comparison with the Monte Carlo simulation results. Lee et al. (2006) proposed a FE rotor model considering a shock base excitation with the state-space Newmark method based on the average velocity concept. They focused also on the experimental behavior of a rotor under this shock base excitation and compared it with the numerical results.

Some other works incorporated the foundation flexibility into the rotordynamics prediction. Kang et al. (2000) studied the effect of the flexible support (foundation) on the dynamic characteristics of the rotor-bearing systems using a FE modeling. Edwards et al. (2000) verified experimentally a method which identifies both the excitation and the flexible support parameters of a rotor-bearing-foundation system. Cavalca et al. (2005) estimated experimentally the modal parameters of the foundation from frequency response functions and studied the effect of the flexible foundation on the dynamic characteristics of the rotor-bearing systems. The experimental tests of Feng and Hahn (2011) showed that even with input data truncated to two significant digits, satisfactory identification were possible for a flexibly supported undamped rigid block foundation in rotating machinery.

A few number of works deal with the investigation of dynamic behavior of a rotor during a harmonic motion of its support. Tan et al. (1997) developed the equations of motion of a rotating cantilever beam induced by a base excitation by employing the Euler-Bernoulli beam theory. They used the method of multiple scales for determining the instability regions and presented numerical results to illustrate the influence of the hub radius to length ratio, the steady-state rotational speed and the base excitation frequency on the dynamic stability of the system. Duchemin et al. (2006) developed the equations of motion by employing the Rayleigh-Ritz (RR) method to study the basic phenomena of a simple rotor model under a sinusoidal support rotation. They applied the method of multiple scales to observe the motion stability of the rotor and presented experimental results to validate the quasi-analytical study. Driot et al. (2006) obtained two gyroscopic and parametric coupled equations of motion of a rotor induced by a harmonic rotational base movement using the RR method. They described the orbits using two numerical methods (the seventh-eighth-order continuous Runge-Kutta method and the normal form method). From their work, the comparison between the numerical and experimental results was quite satisfactory. El-Saeidy and Sticher (2010) derived the equations of motion of a rigid rotor linear/non-linear bearing system subjected to rotating mass unbalance plus harmonic base excitations along or around lateral directions. In the case of linear bearings, they presented analytical solutions and obtained the system natural frequencies as a function of the speed of rotation of the rotor in addition to the frequency response (maximum amplitude of vibrations with respect to the base excitation frequency). In the case of a bearing cubic nonlinearity, they found results from a time integration scheme and discussed them with regards to the time domain, the fast Fourier transform as well as the Poincaré map. Das et al. (2010) investigated the active vibration control of a flexible rotor system excited by a mass unbalance and a periodic rotational motion of the base. They performed the control with electromagnetic control force provided by an actuator placed on the stator in a suitable plane around the rotor. As shown in their paper, a numerical simulation of the flexible rotor system modeled by Rayleigh beam FEs was performed and the active control was successful for avoiding the lateral parametric instability due to the sinusoidal rotation of the base. 
Most of the works mentioned above dealing with base-excited rotor systems are based on a simplified modeling. Thus they limit the satisfactory adequacy between the theoretical rotor models and the realistic ones under operating conditions. The most common simplifications concern either the geometry of the rotor (for example, the Jeffcott rotor (Hori and Kato, 1990)), the use of the RR method instead of the FE method for the spatial discretization of the rotor model (Duchemin et al., 2006; Driot et al., 2006) or the type of base motion (simple rotation or translation).

The present paper proposes an original contribution to improve an on-board rotor model. Namely, a rotor with geometric asymmetry due to the disk and/or the shaft is discretized using the FE method based on the Timoshenko beam theory and excited by combined deterministic base motions (three rotations and three translations). The vibration analysis focuses on the case of bending of the flexible rotor. Eulerian angles are thus used to describe the orientation of disk and shaft with respect to the moving rigid base. The kinetic and strain energies of the rotating flexible rotor components are calculated. Timoshenko beam FEs including the effects relative to the rotary inertia, the gyroscopic inertia, the shear deformation of shaft as well as the geometric asymmetry of disk and/or shaft are considered for the dynamic modeling of the on-board rotor. Applying the Lagrange's equations, the linear second-order differential equations of motion due to the flexural vibration of the rotor relative to the rigid base (which forms a non-inertial coordinate system) are derived. They contain periodic parametric coefficients relative to the geometric asymmetry of some rotor components (disk and shaft) and timevarying parametric coefficients corresponding to the three rotations of the rotor base. These parametric coefficients can be a source of lateral dynamic instability. In the proposed numerical examples, symmetric and asymmetric rotating rotors, whose rigid support is subjected to combined constant angular velocity and harmonic translation, are studied. Their dynamic behavior is analytically and numerically investigated by discussing the influence of the base motions on stability charts, Campbell diagrams, steady-state responses and orbits of the rotor.

\section{Basic description and assumptions}

Let Figure 1 sketch the basic components of the rotor: disk (1), shaft (2), mass unbalance (3), bearing (4) and support (5). The mass unbalance is distributed continuously as well as in a generic way and cannot completely be canceled. The excitations taken into account are due to the residual mass unbalance and to the imposed movements of the support. The transverse deflections of the elastic line of the rotor shaft are studied.

Let the assumptions be: the disk is rigid, the shaft is deformable and modeled with Timoshenko beam elements of constant cross-section solicited in bending along two orthogonal directions, the bearing supporting the shaft is rigid, the disk and/or the shaft can be asymmetric, the mass unbalance is modeled by concentrated masses, the support is rigid and mobile, the rotor runs at a constant speed of rotation $\Omega$.

The consideration of the base motion modifies the form of the equations of motion of a rotor in bending compared to those obtained in the case of a fixed base. In order to undertake a modeling as simple as possible, the approach presented by Duchemin et al. (2006) is adopted. Three frames of reference are used to take into account the movement of the rotor with respect to the base and that of the base with respect to the ground.

The derivation of the equations of motion is inspired from the theory described in Lalanne and Ferraris (1998). First, the coordinate systems needed to describe the motion of an on-board rotor are defined and the vectors expressing the rotations between them are calculated. Then, the kinetic energy of a disk, the kinetic and strain energies of a shaft element as well as the kinetic energy of a concentrated mass unbalance are calculated. Moreover, the rotating flexible rotor is discretized using the Timoshenko beam FEs. Finally, the differential equations of motion of an on-board rotor in bending are deduced from the Lagrange's equations being applied with respect to the generalized coordinates $q_{i}$ by the following form

$$
\frac{d}{d t}\left(\frac{\partial T}{\partial \dot{q}_{i}}\right)-\frac{\partial T}{\partial q_{i}}+\frac{\partial U}{\partial q_{i}}=F_{q_{i}},
$$

with $n_{d f}\left(1 \leq i \leq n_{d f}\right)$ the number of degrees of freedom, $T$ and $U$ the kinetic and strain energies respectively and $F_{q_{i}}$ the external forces corresponding to $q_{i}$. The symbol "•" refers to the differentiation with respect to time $t$

\section{Preliminary calculations}

The utilization of more than one frame of reference needs to establish the relationships between the different defined systems. Three principal Cartesian frames of reference shown in Figure 1 are introduced to take into account the mobility of the rotor support: $R^{g}\left(x^{g}, y^{g}, z^{g}\right)$ is the Galilean frame of reference fixed to the ground, $R(x, y, z)$ is the non-inertial frame of reference attached to the moving rigid support, $R^{l}\left(x^{l}, y^{l}, z^{l}\right)$ is the noninertial local frame of reference (moving with the rotor during its operation) attached to the center of mass of the rigid disk or the geometric center of a section of the deformable shaft. The origins of the frames of reference $R^{g}, R$ and $R^{l}$ are $O^{g}, O$ and $O^{l}$ respectively. 
The elastic line of the undeformed shaft is along the $O y$ axis on which the disk mass center is located. Moreover, $x O y$ and $y O z$ are both planes of symmetry for the disk and the undeformed shaft. Thus $O x, O y$ and $O z$ are principal axes of inertia.

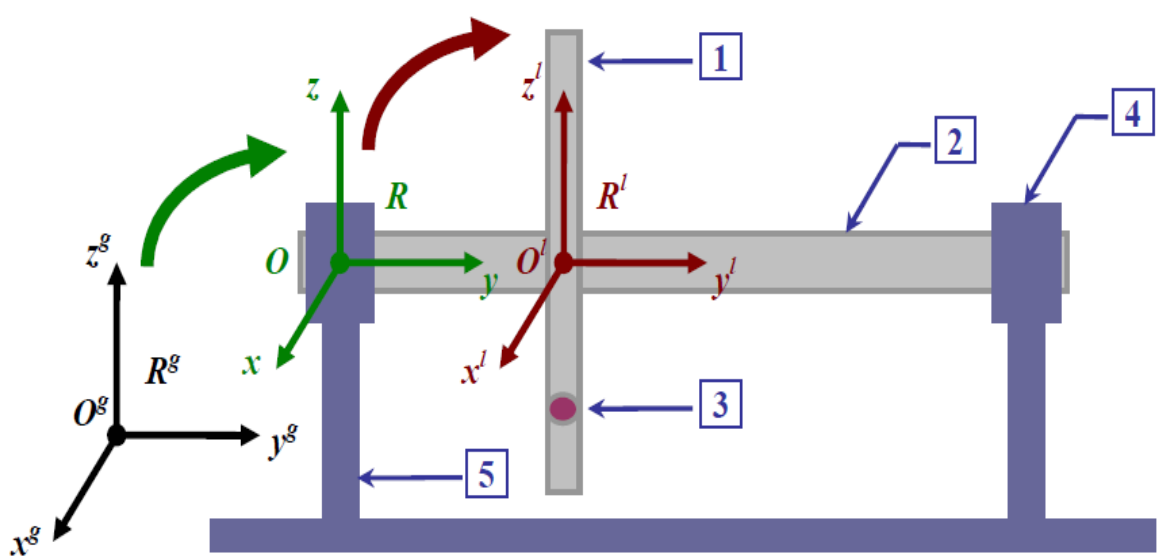

Figure 1. Mechanical components and frames of reference of the on-board rotor model.

Let us consider an arbitrary point $C^{0}$ along the elastic line, i.e., it represents the geometric center of the undeformed shaft. Its coordinates in the frame $R$ are $(0, y, 0)$. Let its lateral displacements along the $O x$ and $O z$ axes be $u(y, t)$ and $w(y, t)$, see Figure 2. As its coordinate along the $O y$ axial axis is assumed to be a constant with respect to the rotor base $R$, the axial displacement along the $O y$ axis is not taken into account.

In classical rotordynamics theory, the transformation between the frames $R$ and $R^{l}$ shown in Figure 3 is performed by the Euler angles $\psi(y, t), \theta(y, t), \phi(t)$ and two intermediate coordinate systems $R^{i_{1}}\left(x^{i_{1}}, y^{i_{1}}, z^{i_{1}}\right)$ and $R^{i_{2}}\left(x^{i_{2}}, y^{i_{2}}, z^{i_{2}}\right)$. The relationship between the components of any vector $\mathbf{V}$ expressed in $R$ and those expressed in $R^{l}$ is described by

$$
\left.\mathbf{V}\right|_{R^{l}}=\left.\Re_{R^{l}}^{R} \mathbf{V}\right|_{R}
$$

where

$$
\mathfrak{R}_{R^{l}}^{R}=\left[\begin{array}{ccc}
\cos \psi \cos \phi-\sin \psi \sin \theta \sin \phi & \sin \psi \cos \phi+\cos \psi \sin \theta \sin \phi & -\cos \theta \sin \phi \\
-\sin \psi \cos \theta & \cos \psi \cos \theta & \sin \theta \\
\cos \psi \sin \phi+\sin \psi \sin \theta \cos \phi & \sin \psi \sin \phi-\cos \psi \sin \theta \cos \phi & \cos \theta \cos \phi
\end{array}\right],
$$

is the rotation matrix of the rotor $R^{l}$ with respect to its base $R$.

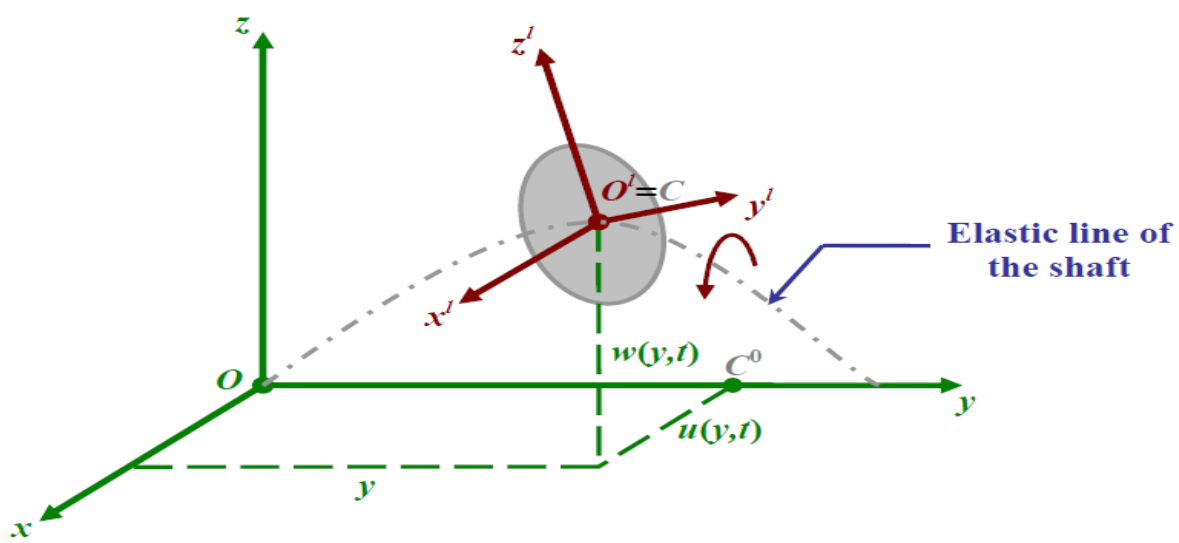

Figure 2. Lateral displacements of the rotor in bending. 


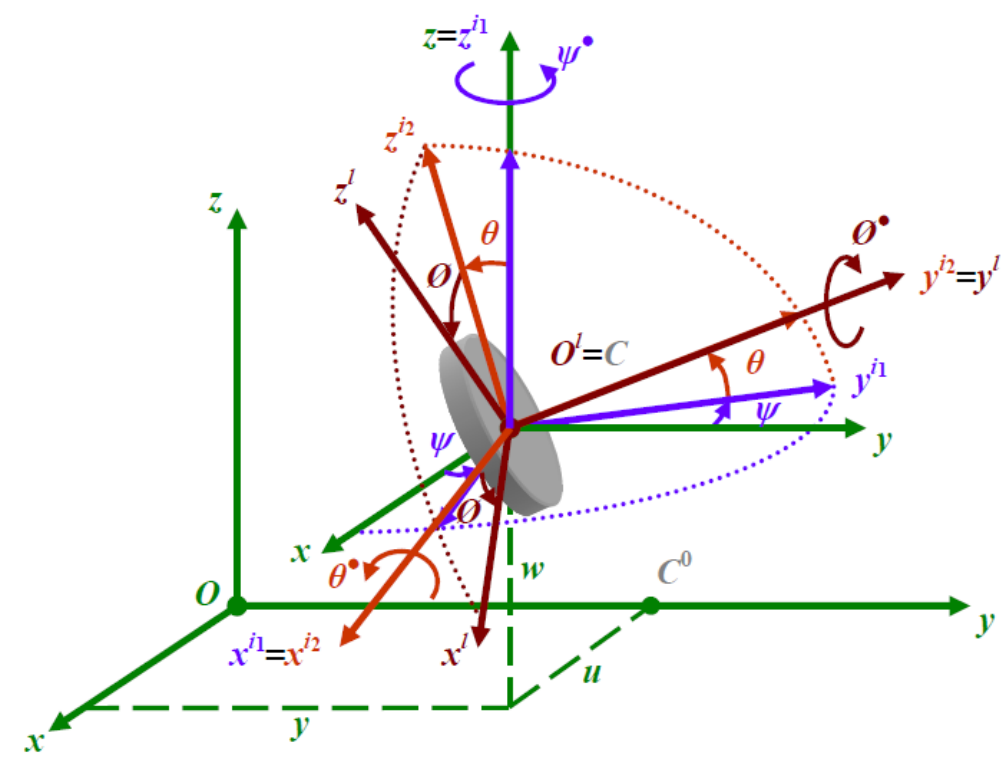

Figure 3. Passage from the frame linked with the rotor base $R$ to the local frame $R$.

Using the intermediate coordinate systems $R^{i_{3}}\left(x^{i_{3}}, y^{i_{3}}, z^{i_{3}}\right)$ and $R^{i_{4}}\left(x^{i_{4}}, y^{i_{4}}, z^{i_{4}}\right)$ as well as the Euler angles $\alpha(t), \beta(t)$ and $\gamma(t)$, see Figure 4, the relationship between the components of any vector $\mathbf{V}$ projected in $R^{g}$ and those projected in $R$ is expressed as follows

$$
\left.\mathbf{V}\right|_{R}=\left.\Re_{R}^{R^{g}} \mathbf{V}\right|_{R^{g}},
$$

where

$$
\mathfrak{R}_{R}^{R^{g}}=\left[\begin{array}{ccc}
\cos \alpha \cos \gamma-\sin \alpha \sin \beta \sin \gamma & \sin \alpha \cos \gamma+\cos \alpha \sin \beta \sin \gamma & -\cos \beta \sin \gamma \\
-\sin \alpha \cos \beta & \cos \alpha \cos \beta & \sin \beta \\
\cos \alpha \sin \gamma+\sin \alpha \sin \beta \cos \gamma & \sin \alpha \sin \gamma-\cos \alpha \sin \beta \cos \gamma & \cos \beta \cos \gamma
\end{array}\right],
$$

is the rotation matrix of the rotor base $R$ with respect to the ground $R^{g}$.

Computing the energies of the rotor components requires establishing the angular velocity vectors of the coordinate systems and the position vectors of their origins. The instantaneous angular velocity vector $\boldsymbol{\omega}_{R^{l}}^{R}$ of the frame $R^{l}$ with respect to the frame $R$ is written as

$$
\omega_{R^{l}}^{R}=\dot{\theta} \mathbf{x}^{\mathrm{i}_{1}}+\dot{\phi} \mathbf{y}^{1}+\dot{\psi} \mathbf{z}
$$

where $\mathbf{x}^{\mathrm{i}_{1}}, \mathbf{y}^{\mathbf{l}}$ and $\mathbf{z}$ are unit vectors along the $O^{l} x^{i_{1}}, O^{l} y^{l}$ and $O^{l} z$ axes. Let $\omega_{1}^{x^{l}}, \omega_{1}^{y^{l}}$ and $\omega_{1}^{z^{l}}$ be the components of the angular velocity vector $\omega_{R^{l}}^{R}$ projected in the local frame $R^{l}$. Their expression is given by

$$
\boldsymbol{\omega}_{R^{l}}^{R}=\left\{\begin{array}{c}
\omega_{1}^{x^{l}} \\
\omega_{1}^{y^{l}} \\
\omega_{1}^{z^{l}}
\end{array}\right\}_{R^{l}}=\left\{\begin{array}{c}
-\dot{\psi} \cos \theta \sin \phi+\dot{\theta} \cos \phi \\
\dot{\psi} \sin \theta+\dot{\phi} \\
\dot{\psi} \cos \theta \cos \phi+\dot{\theta} \sin \phi
\end{array}\right\}_{R^{l}} .
$$

As before, the instantaneous angular velocity vector $\boldsymbol{\omega}_{R}^{R^{g}}$ which achieves the orientation of the frame attached to the rotor base $R$ with respect to the Galilean frame $R^{g}$ is expressed in the frame $R$ by

$$
\boldsymbol{\omega}_{R}^{R^{g}}=\left\{\begin{array}{c}
\omega^{x} \\
\omega^{y} \\
\omega^{z}
\end{array}\right\}_{R}=\left\{\begin{array}{c}
-\dot{\alpha} \cos \beta \sin \gamma+\dot{\beta} \cos \gamma \\
\dot{\alpha} \sin \beta+\dot{\gamma} \\
\dot{\alpha} \cos \beta \cos \gamma+\dot{\beta} \sin \gamma
\end{array}\right\}_{R},
$$

where $\omega^{x}, \omega^{y}$ and $\omega^{z}$ are the components of the angular velocity vector $\omega_{R}^{R^{g}}$ defined in the coordinate system fixed to the base $R$. Let $\omega_{2}^{x^{l}}, \omega_{2}^{y^{l}}$ and $\omega_{2}^{z^{l}}$ be the components of the angular velocity vector $\boldsymbol{\omega}_{R}^{R^{g}}$ of the rigid base $R$ with respect to the ground $R^{g}$ projected in the local frame $R^{l}$. Their expression is obtained by equations (2) and (3) defining the relationship between the frames $R$ and $R^{l}$, i.e.,

$$
\boldsymbol{\omega}_{R}^{R^{g}}=\left\{\begin{array}{c}
\omega_{2}^{x^{l}} \\
\omega_{2}^{y^{l}} \\
\omega_{2}^{z^{l}}
\end{array}\right\}_{R^{l}}=\Re_{R^{l}}^{R}\left\{\begin{array}{l}
\omega^{x} \\
\omega^{y} \\
\omega^{z}
\end{array}\right\}_{R} .
$$




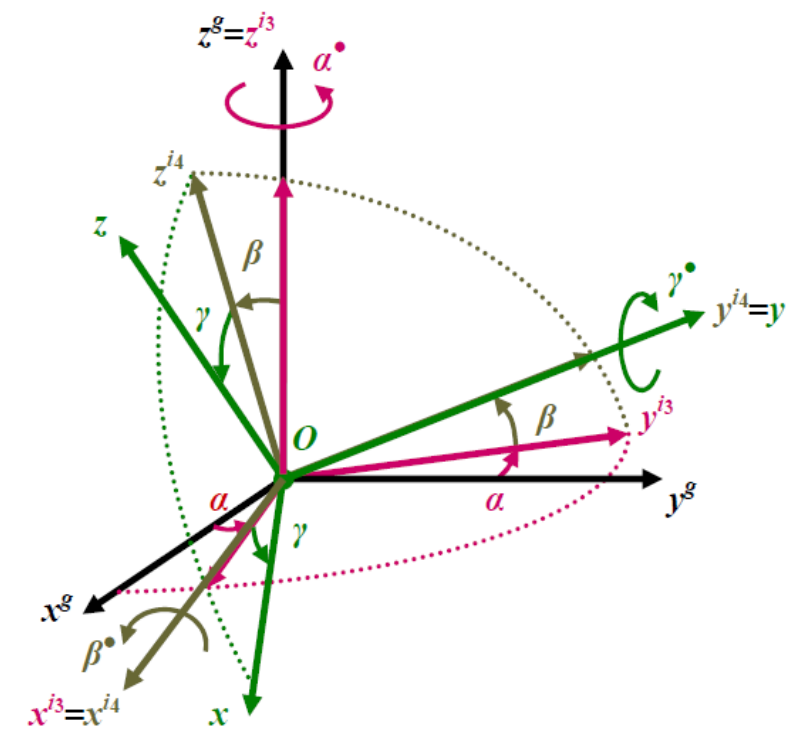

Figure 4. Passage from the Galilean frame $R^{g}$ to the frame linked with the rotor base $R$.

Finally, the instantaneous angular velocity vector $\omega_{R^{l}}^{R^{g}}$ of the rotor $R^{l}$ with respect to the ground $R^{g}$ expressed in the local coordinate system $R^{l}$ represents the sum of the vectors $\boldsymbol{\omega}_{R^{l}}^{R}$ and $\boldsymbol{\omega}_{R}^{R^{g}}$ presented in equations (7) and (9), then

$$
\boldsymbol{\omega}_{R^{l}}^{R^{g}}=\boldsymbol{\omega}_{R^{l}}^{R}+\boldsymbol{\omega}_{R}^{R^{g}}=\left\{\begin{array}{c}
\omega_{1}^{x^{l}} \\
\omega_{1}^{y^{l}} \\
\omega_{1}^{z^{l^{l}}}
\end{array}\right\}_{R^{l}}+\left\{\begin{array}{c}
\omega_{2}^{x^{l}} \\
\omega_{2}^{y^{l}} \\
\omega_{2}^{z^{l}}
\end{array}\right\}_{R^{l}}=\left\{\begin{array}{c}
\omega^{x^{l}} \\
\omega^{y^{l}} \\
\omega^{z^{l}}
\end{array}\right\}_{R^{l}} .
$$

The absolute position vector $\mathbf{O}^{\mathrm{g}} \mathbf{O}$ of the origin $O$ of the frame attached to the base $R$ expressed in the Galilean frame of reference $R^{g}$ is given by

$$
\mathbf{O}^{\mathrm{g}} \mathbf{O}=\left\{\begin{array}{c}
x_{o}^{g} \\
y_{O}^{g} \\
z_{o}^{g}
\end{array}\right\}_{R^{g}} .
$$

Using equations (4) and (5) expressing the relationship between the frames $R^{g}$ and $R$, the position vector $\mathbf{O}^{\mathrm{g}} \mathbf{O}$ is expressed in the frame $R$ as

$$
\mathbf{O}^{\mathrm{g}} \mathbf{O}=\left\{\begin{array}{l}
x_{O} \\
y_{O} \\
z_{O}
\end{array}\right\}_{R}=\boldsymbol{\Re}_{R}^{R^{g}}\left\{\begin{array}{c}
x_{O}^{g} \\
y_{O}^{g} \\
z_{O}^{g}
\end{array}\right\}_{R^{g}} .
$$

In what follows, the calculations are performed using the rotational $\omega^{x}(t), \omega^{y}(t), \omega^{z}(t)$ and the translational $x_{O}(t), y_{O}(t), z_{O}(t)$ base motion components as well as their derivatives with respect to time $t$.

\section{Energy calculations of the rotor components}

This section includes formulations for the disk, the shaft and the rotating mass unbalance. The kinetic and strain energies are measured by an observer located at the ground and their terms are written with respect to the frame linked with the rotor rigid base $R$. The rotatory inertia, the gyroscopic inertia, the shear deformation of shaft as well as the geometric asymmetry of disk and/or shaft are taken into consideration.

\subsection{Disk}

According to the assumptions presented in Section 2, the disk is assumed to be rigid. Therefore only its kinetic energy $T_{d}$ is calculated. This energy is composed of the scalar sum of the absolute translational kinetic energy and the absolute rotational kinetic energy about the disk mass center located at the generic abscissa $y_{d}$ along the Oy axis

$$
T_{d}=\frac{m_{d}}{2}\left(\mathbf{v}_{O_{d}^{l}}^{R^{g}}\right)^{T} \mathbf{v}_{O_{d}^{l}}^{R^{g}}+\frac{1}{2}\left(\boldsymbol{\omega}_{R^{l}}^{R^{g}}\right)^{T} \mathbf{I}_{m_{d}} \boldsymbol{\omega}_{R^{l}}^{R^{g}}
$$

with 


$$
\mathbf{I}_{m_{d}}=\left[\begin{array}{ccc}
I_{m_{d}}^{x} & 0 & 0 \\
0 & I_{m_{d}}^{y} & 0 \\
0 & 0 & I_{m_{d}}^{z}
\end{array}\right]=\left[\begin{array}{ccc}
I_{m_{d}}^{m o}+I_{m_{d}}^{d i} & 0 & 0 \\
0 & I_{m_{d}}^{y} & 0 \\
0 & 0 & I_{m_{d}}^{m o}-I_{m_{d}}^{d i}
\end{array}\right],
$$

where the superscript $T$ is the matrix transposition symbol, $m_{d}$ is the mass of the disk, $\mathbf{v}_{O_{d}^{l}}^{R^{g}}$ is the translational velocity vector of its center and $\mathbf{I}_{m_{d}}$ is its principal inertia tensor. $I_{m_{d}}^{x}, I_{m_{d}}^{y}$ and $I_{m_{d}}^{z}$ are the principal moments of inertia of the disk mass about the $C^{0} x, C^{0} y$ and $C^{0} z$ axes respectively. In addition, $I_{m_{d}}^{m o}$ and $I_{m_{d}}^{d i}$ are used to explain the effects due to the mean moment of inertia of the disk mass and those due to the moment of inertia modeling the geometric asymmetry of the disk. Thus in the presence of an asymmetry, the moments of inertia $I_{m_{d}}^{x}$ and $I_{m_{d}}^{z}$ are different and the contribution of the moment of inertia $I_{m_{d}}^{d i}$ in them is not nil.

The translational velocity vector $\mathbf{v}_{O_{d}^{l}}^{R^{g}}$ is found through the absolute position vector $\mathbf{O}^{\mathrm{g}} \mathbf{O}_{\mathrm{d}}^{1}$ of the disk mass center positioned at the arbitrary abscissa $y_{d}$ relative to the frame $R$. The latter is observed from the ground and expressed in the frame attached to the rotor base $R$ by

$$
\mathbf{O}^{g} \mathbf{O}_{\mathbf{d}}^{1}=\mathbf{O}^{\mathrm{g}} \mathbf{O}+\mathbf{O} \mathbf{O}_{\mathbf{d}}^{1}=\left\{\begin{array}{l}
x_{o} \\
y_{O} \\
z_{o}
\end{array}\right\}_{R}+\left\{\begin{array}{l}
u_{d} \\
y_{d} \\
w_{d}
\end{array}\right\}_{R}=\left\{\begin{array}{l}
u_{d}+x_{o} \\
y_{d}+y_{o} \\
w_{d}+z_{o}
\end{array}\right\} .
$$

Using the transport theorem relating the time derivative of a vector measured from a fixed frame to that of the same vector but measured from a moving frame, the derivation of the position vector $\mathbf{O}^{\mathrm{g}} \mathbf{O}_{\mathrm{d}}^{1}$ yields

$$
\frac{d \mathbf{O}^{\mathrm{g}} \mathbf{O}_{\mathbf{d}}^{R^{g}}}{d t}=\frac{d \mathbf{O}^{\mathrm{g}} \mathbf{O}_{\mathbf{d}}^{1^{R}}}{d t}+\boldsymbol{\omega}_{R}^{R^{8}} \wedge \mathbf{O}^{\mathrm{g}} \mathbf{O}_{\mathbf{d}}^{1}=\mathbf{v}_{O_{d}^{\prime}}^{R^{8}},
$$

then

$$
\mathbf{v}_{O_{d}^{\prime}}^{R^{g}}=\left\{\begin{array}{c}
\dot{u}_{d}+\dot{x}_{o}+\left(w_{d}+z_{o}\right) \omega^{y}-\left(y_{d}+y_{o}\right) \omega^{z} \\
\dot{y}_{O}-\left(w_{d}+z_{o}\right) \omega^{x}+\left(u_{d}+x_{o}\right) \omega^{z} \\
\dot{w}_{d}+\dot{z}_{o}+\left(y_{d}+y_{O}\right) \omega^{x}-\left(u_{d}+x_{o}\right) \omega^{y}
\end{array}\right\}=\left\{\begin{array}{c}
\dot{u}_{O_{d}^{\prime}} \\
\dot{v}_{o_{d}^{d}} \\
\dot{w}_{o_{d}^{\prime}}
\end{array}\right\}_{R},
$$

where $\dot{u}_{o_{d}^{l}}, \dot{v}_{o_{d}^{l}}$ and $\dot{w}_{o_{d}^{l}}$ are the components of the translational velocity vector of the disk center. Considering the components $\omega_{d}^{x^{l}}, \omega_{d}^{y^{l}}$ and $\omega_{d}^{z^{l}}$ of the vector $\boldsymbol{\omega}_{R^{l}}^{R^{8}}$ for the disk, the expression of its kinetic energy becomes

$$
T_{d}=\frac{m_{d}}{2}\left(\dot{u}_{O_{d}^{l}}^{2}+\dot{v}_{O_{d}^{l}}^{2}+\dot{w}_{O_{d}^{l}}^{2}\right)+\frac{1}{2}\left(I_{m_{d}}^{m o}\left(\omega_{d}^{x^{l} 2}+\omega_{d}^{z^{l} 2}\right)+I_{m_{d}}^{y} \omega_{d}^{y^{y^{l} 2}}+I_{m_{d}}^{d i}\left(\omega_{d}^{x^{l} 2}-\omega_{d}^{z^{l^{2}} 2}\right)\right) .
$$

The spinning angle $\phi$ is replaced by $\Omega t$ and its time derivative $\dot{\phi}$ by the constant speed of rotation $\Omega$ of the rotor. The translational displacements $\left(u_{d}, w_{d}\right)$, the angles of rotation $\left(\psi_{d}, \theta_{d}\right)$ and their time derivatives are very small. Thus the trigonometric functions are replaced by their Taylor series expansion and the resulting expressions are limited at the order 2 , i.e.,

$$
\begin{aligned}
& \sin \theta_{d} \simeq \theta_{d} \quad ; \quad \cos \theta_{d} \simeq 1-\frac{\theta_{d}^{2}}{2}, \\
& \sin \psi_{d} \simeq \psi_{d} \quad ; \quad \cos \psi_{d} \simeq 1-\frac{\psi_{d}^{2}}{2} .
\end{aligned}
$$

\subsection{Shaft}

The kinetic energy of the shaft element can be obtained by taking a shaft elementary volume which can be considered as a disk of very small thickness $d y$. Considering the components $\dot{u}_{O^{l}}, \dot{v}_{O^{l}}, \dot{w}_{O^{l}}$ of the vector $\mathbf{v}_{O^{l}}^{R^{g}}$ and the components $\omega^{x^{l}}, \omega^{y^{l}}, \omega^{z^{l}}$ of the vector $\boldsymbol{\omega}_{R^{l}}^{R^{g}}$, the expression for the kinetic energy $T_{s h}$ of the shaft element, whose end-points have the abscissas $y_{1}$ and $y_{2}$ along the $O y$ axis, is written in the following form

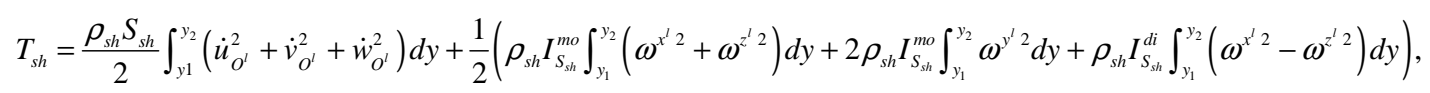

where $\rho_{s h}, S_{s h}$ and $l_{s h}\left(l_{s h}=y_{2}-y_{1}\right)$ are respectively the density, the cross-sectional area and the length of the shaft element. The mean moment of inertia of the cross-sectional area and the inertia characterizing the asymmetry of the shaft are

$$
I_{S_{s h}}^{m o}=\frac{\left(I_{S_{s h}}^{x}+I_{S_{s h}}^{z}\right)}{2} ; I_{S_{s h}}^{d i}=\frac{\left(I_{S_{s h}}^{x}-I_{S_{s h}}^{z}\right)}{2}
$$


The rigid base motion relative to the ground has no influence on the strain energy of the shaft because the latter depends only on the stresses and therefore on the transverse deflection of the shaft with respect to the rotor base $R$. In addition to the bending deformation, the shear effects highlighted by Timoshenko and the secondorder (non-linear) terms of the strain field are accounted. The non-linear strains are linked with the centrifugal stiffening which is proportional to the square of the base angular velocity around the transverse axes and which corresponds to the geometric potential energy. Thus the strain energy $U_{s h}$ of the shaft element is defined by

$$
\begin{aligned}
U_{s h} & =\frac{E_{s h} I_{S_{s h}}^{m o}}{2} \int_{y_{1}}^{y_{2}}\left(\left(\frac{\partial \psi}{\partial y}\right)^{2}+\left(\frac{\partial \theta}{\partial y}\right)^{2}\right) d y+\frac{G_{s h} K_{s h}^{m o} S_{s h}}{2} \int_{y_{1}}^{y_{2}}\left(\left(\frac{\partial u}{\partial y}+\psi\right)^{2}+\left(\frac{\partial w}{\partial y}-\theta\right)^{2}\right) d y-\frac{1}{2}\left(E_{s h} I_{s s h}^{d i} \int_{y_{1}}^{y_{2}}\left(\left(\frac{\partial \psi}{\partial y}\right)^{2}-\left(\frac{\partial \theta}{\partial y}\right)^{2}\right) d y\right. \\
& \left.-G_{s h} \kappa_{s h}^{d i} S_{s h} \int_{y_{1}}^{y_{2}}\left(\left(\frac{\partial u}{\partial y}+\psi\right)^{2}-\left(\frac{\partial w}{\partial y}-\theta\right)^{2}\right) d y\right) \cos 2 \Omega t-\left(E_{s h} I_{s s h}^{d i} \int_{y_{1}}^{y_{2}} \frac{\partial \psi}{\partial y} \frac{\partial \theta}{\partial y} d y\right. \\
& \left.-G_{s h} \kappa_{s h}^{d i} S_{s h} \int_{y_{1}}^{y_{2}}\left(\frac{\partial u}{\partial y}+\psi\right)\left(\frac{\partial w}{\partial y}-\theta\right) d y\right) \sin 2 \Omega t+\frac{\rho_{s h} S_{s h}}{4} \int_{y_{1}}^{y_{2}}\left(\left(l_{s h}+y_{1}\right)^{2}-y^{2}\right)\left(\left(\frac{\partial u}{\partial y}\right)^{2}+\left(\frac{\partial w}{\partial y}\right)^{2}\right) d y\left(\omega^{x 2}+\omega^{z 2}\right),
\end{aligned}
$$

with

$$
G_{s h}=\frac{E_{s h}}{2\left(1+v_{s h}\right)} \quad ; \quad \kappa_{s h}^{m o}=\frac{\kappa_{s h}^{x}+\kappa_{s h}^{z}}{2} ; \quad \kappa_{s h}^{d i}=\frac{\kappa_{s h}^{x}-\kappa_{s h}^{z}}{2},
$$

where $E_{s h}, G_{s h}$ and $v_{s h}$ are respectively the Young's modulus, the shear modulus and the Poisson's ratio of the isotropic shaft material. $\kappa_{s h}^{m o}$ and $\kappa_{s h}^{d i}$ are respectively the mean shear correction factor of the cross-sectional area and that relative to the section asymmetry of the shaft.

\subsection{Mass unbalance}

The mass unbalance is modeled by a concentrated mass $m_{m и}$ placed at a point $P_{m и}$ with a distance $r_{m u}$ from the geometric center of the cross-sectional area of the shaft. Its initial angle with the $O z$ axis of the frame $R$ at rest is $\eta_{m u}$. The mass unbalance remains in a plane perpendicular to the $O y$ axis with a constant abscissa $y_{m u}$ along the $O y$ axis relative to the base $R$. Its kinetic energy $T_{m u}$ is expressed as follows

$$
T_{m u}=\frac{m_{m u}}{2}\left(\mathbf{v}_{P_{m u}}^{R^{g}}\right)^{T} \mathbf{v}_{P_{m u}}^{R^{g}} \text {. }
$$

The translational velocity vector $\mathbf{v}_{P_{m u}}^{R^{g}}$ is obtained using the absolute position vector $\mathbf{O}^{\mathbf{g}} \mathbf{P}_{\mathrm{mu}}$ of the mass unbalance. The latter is measured from the ground and written relative to the frame linked with the base $R$ as

$$
\mathbf{O}^{\mathbf{g}} \mathbf{P}_{\mathrm{mu}}=\mathbf{O}^{\mathrm{g}} \mathbf{O}+\mathbf{O O}_{\mathrm{mu}}^{1}+\mathbf{O}_{\mathrm{mu}}^{1} \mathbf{P}_{\mathrm{mu}}=\left\{\begin{array}{c}
u_{m u}+x_{O}+r_{m u} \sin \left(\Omega t+\eta_{m u}\right) \\
y_{m u}+y_{O} \\
w_{m u}+z_{O}+r_{m u} \cos \left(\Omega t+\eta_{m u}\right)
\end{array}\right\}_{R} .
$$

Applying the transport theorem, the time derivative of the position vector $\mathbf{O}^{\mathrm{g}} \mathbf{P}_{\mathrm{mu}}$ is of the following form

$$
\begin{aligned}
& \frac{d \mathbf{O}^{\mathbf{g}} \mathbf{P}_{\mathrm{mu}}{ }^{R^{g}}}{d t}=\frac{d \mathbf{O}^{\mathbf{g}} \mathbf{P}_{\mathrm{mu}}{ }^{R}}{d t}+\boldsymbol{\omega}_{R}^{R^{g}} \wedge \mathbf{O}^{\mathbf{g}} \mathbf{P}_{\mathrm{mu}}=\mathbf{v}_{P_{m u}{ }^{g}}, \\
& \mathbf{v}_{P_{m u}}^{R^{g}}=\left\{\begin{array}{c}
\dot{u}_{m u}+\dot{x}_{O}+r_{m u} \Omega \cos \left(\Omega t+\eta_{m u}\right)+\left(w_{m u}+z_{O}+r_{m u} \cos \left(\Omega t+\eta_{m u}\right)\right) \omega^{y}-\left(y_{m u}+y_{O}\right) \omega^{z} \\
\dot{y}_{O}-\left(w_{m u}+z_{O}+r_{m u} \cos \left(\Omega t+\eta_{m u}\right)\right) \omega^{x}+\left(u_{m u}+x_{O}+r_{m u} \sin \left(\Omega t+\eta_{m u}\right)\right) \omega^{z} \\
\dot{w}_{m u}+\dot{z}_{O}-r_{m u} \Omega \sin \left(\Omega t+\eta_{m u}\right)+\left(y_{m u}+y_{O}\right) \omega^{x}-\left(u_{m u}+x_{O}+r_{m u} \sin \left(\Omega t+\eta_{m u}\right)\right) \omega^{y}
\end{array}\right\}_{R} .
\end{aligned}
$$

The external force vectors obtained by the application of the Lagrange's equations to the kinetic energy of the mass unbalance contain the so-called "centripetal and centrifugal force vectors", harmonic tangential force vectors and harmonic force vectors fixed in space with respect to the base $R$. Thus the excitation due to the mass unbalance combined with the base rotations is sinusoidal and has a frequency equal to the speed of rotation $\Omega$ of the rotor.

\section{Equations of motion of an on-board rotor}

\subsection{Finite element procedure}

The FE analysis is selected to discretize the rotor in bending. Therefore each node of nodal interpolation contains four degrees of freedom (two lateral translations and two rotations). The disk and the mass unbalance are modeled by one-node FEs situated at the geometric center of the cross-sectional area of the shaft. On the other hand, the shaft is modeled by $n_{\text {esh }}$ two-node beam FEs based on the Timoshenko beam theory and thus has $n_{e s h}+1$ nodes. Let us consider an undeformed shaft FE esh $h_{i}$ which consists of two nodes $n_{i}$ and $n_{i+1}$ of coordinates $y_{s h}^{n_{i}}$ and $y_{s h}^{n_{i+1}}$ along the $O y$ axis. This FE is of density $\rho_{e s h_{i}}$, cross-sectional area $S_{e s h_{i}}$, length $l_{e s h_{i}}$, 
moments of inertia $I_{S_{e s h_{i}}^{m o}}^{m o}$ and $I_{S_{e s h_{i}}}^{d i}$, Young's modulus $E_{e s h_{i}}$, Poisson's ratio $v_{e s h_{i}}$ as well as shear correction factors $\kappa_{e s h_{i}}^{m o}$ and $\kappa_{e s h_{i}}^{d i}$ where $i\left(1 \leq i \leq n_{e s h}\right)$ is the shaft FE number. Its nodal displacement vector $\boldsymbol{\delta}_{e s h_{i}}^{n}$ expressed in the coordinate system attached to the rotor rigid base $R$ is given by

$$
\boldsymbol{\delta}_{e s h_{i}}^{n}=\left\langle u_{e s h_{i}}^{n_{i}}, w_{e s h_{i}}^{n_{i}}, \theta_{e s h_{i}}^{n_{i}}, \psi_{e s h_{i}}^{n_{i}}, u_{e s h_{i}}^{n_{i+1}}, w_{e s h_{i}}^{n_{i+1}}, \theta_{e s h_{i}}^{n_{i+1}}, \psi_{e s h_{i}}^{n_{i+1}}\right\rangle_{R}^{T} .
$$

The above vector is separated into two vectors $\boldsymbol{\delta}_{e s h_{i}}^{n, u}$ and $\boldsymbol{\delta}_{e s h_{i}}^{n, w}$ corresponding to the motions of the nodes $n_{i}$ and $n_{i+1}$ in the planes $x O y$ and $y O z$, i.e.,

$$
\boldsymbol{\delta}_{e s h_{i}}^{n, u}=\left\langle u_{e s h_{i}}^{n_{i}}, \psi_{e s h_{i}}^{n_{i}}, u_{e s h_{i}}^{n_{i+1}}, \psi_{e s h_{i}}^{n_{i+1}}\right\rangle_{R}^{T} \quad ; \quad \boldsymbol{\delta}_{e s h_{i}}^{n, w}=\left\langle w_{e s h_{i}}^{n_{i}}, \theta_{e s h_{i}}^{n_{i}}, w_{e s h_{i}}^{n_{i+1}}, \theta_{e s h_{i}}^{n_{i+1}}\right\rangle_{R}^{T}
$$

The lateral displacements $u_{e s h_{i}}$ and $w_{e s h_{i}}$ of any point $C^{0}$ due to bending respectively along the $O x$ and $O z$ axes of the frame $R$ as well as the corresponding rotations $\theta_{e s h_{i}}$ and $\psi_{\text {esh }}$ respectively around the $O x$ and $O z$ directions are described as a function of the nodal displacement vectors $\boldsymbol{\delta}_{e s h_{i}}^{n, u}$ and $\boldsymbol{\delta}_{e s h_{i}}^{n, w}$ as follows

$$
\begin{aligned}
& u_{e s h_{i}}=\overline{\mathbf{F}_{e s h_{i}}^{u}}(\bar{y}) \boldsymbol{\delta}_{e s h_{i}}^{n, u} ; \psi_{e s h_{i}}=\overline{\mathbf{F}_{e s h_{i}}^{\psi}}(\bar{y}) \boldsymbol{\delta}_{e s h_{i}}^{n, u}, \\
& w_{e s h_{i}}=\overline{\mathbf{F}_{e s h_{i}}^{w}}(\bar{y}) \boldsymbol{\delta}_{e s h_{i}}^{n, w} ; \theta_{e s h_{i}}=\overline{\mathbf{F}_{e s h_{i}}^{\theta}}(\bar{y}) \boldsymbol{\delta}_{e s h_{i}}^{n, w},
\end{aligned}
$$

where $\overline{\mathbf{F}_{e s h_{i}}^{u}}(\bar{y}), \overline{\mathbf{F}_{e s h_{i}}^{\psi}}(\bar{y}), \overline{\mathbf{F}_{e s h_{i}}^{w}}(\bar{y})$ and $\overline{\mathbf{F}_{e s h_{i}}^{\theta}}(\bar{y})$ are the shape function vectors of a Timoshenko beam FE. They are third-degree and second-degree polynomial equations, functions of the dimensionless variable $\bar{y}$ $\left(\bar{y}=y / l_{e s h_{i}}\right)$ along the axis $O y$ and contain the shear effect (see chen and $\left.\mathrm{Ku}, 1992\right)$.

The Lagrange's equations are applied to the energies for the disk, the shaft FEs as well as the mass unbalance (see equations (18), (20), (22) and (24)) and the resulting elementary vectors and matrices are appropriately assembled. In order to deal with the boundary conditions $n_{b c}$, the corresponding row and column entries of the assembled vectors and matrices are set to zero and eliminated. Thus the obtained global vectors and matrices are respectively of dimension $n_{g} \times 1$ and $n_{g} \times n_{g}$ where $n_{g}=4\left(n_{e s h}+1\right)-n_{b c}$ is the total number of degrees of freedom after canceling those relative to the boundary conditions $n_{b c}$. The obtained linear second-order differential equations describing the behavior of the FE on-board rotor in bending are written with respect to the non-inertial frame connected to the rigid base $R$ as follows

$$
\mathbf{M}_{r}(t) \ddot{\boldsymbol{\delta}}_{r}+\mathbf{C}_{r}(t) \dot{\boldsymbol{\delta}}_{r}+\mathbf{K}_{r}(t) \boldsymbol{\delta}_{r}=\mathbf{F}_{r}(t),
$$

where $\boldsymbol{\delta}_{r}, \dot{\boldsymbol{\delta}}_{r}$ and $\ddot{\boldsymbol{\delta}}_{r}$ are the global displacement, velocity and acceleration vectors conforming to the connectivity of the FEs. $\mathbf{M}_{r}(t), \mathbf{C}_{r}(t)$ and $\mathbf{K}_{r}(t)$ are the global mass, damping and stiffness matrices with time-varying parameters due to the geometric asymmetry of the rotating rotor and to the rotations of its moving base. These matrices are not affected by the translational motions of the rotor base. Lastly, $\mathbf{F}_{r}(t)$ is the global external force vector including the excitations due to the influence of the mass unbalance as well as to that of the rotational and translational base motions. In what follows, two different rotor configurations (symmetric and asymmetric) are to be treated and their governing differential equations for the transverse vibrations are presented. The following abbreviations and terms used in the equations are detailed hereafter. The subscripts " $d$ ", "sh" and " $b$ " refer to the disk, the shaft as well as the base respectively and express the contribution to the phenomena represented by the corresponding matrix. The superscript " $i d$ " stands for the rotor internal damping introduced by estimating the Rayleigh damping coefficients, " $g$ " for the rotor gyroscopic effect, " $e$ " for the shaft elasticity corresponding to the bending and shear deformations, "re" for the rotational effects due to the base rotations (these effects come from the kinetic energies of the disk and the shaft) and " gse" for the geometric stiffening effects associated with the centrifugal stressing due to the base rotations (these effects come from the strain energy of the shaft). In addition, the superscripts " $c$ " and " $s$ " denote the coefficients of the timevarying trigonometric (cosine and sine) functions. The load vectors $\mathbf{V}_{m u}, \mathbf{V}_{d, s h, b}$ and $\mathbf{V}_{m u, b}$ and their corresponding force vectors $\mathbf{F}_{m u}, \mathbf{F}_{d, s h, b}$ and $\mathbf{F}_{m u, b}$ are associated respectively with the mass unbalance, the inertia force due to base motions and that due to coupling between both phenomena.

\subsection{Symmetric rotor}

The disk and the shaft are symmetric. Moreover, the rotor is excited by a mass unbalance and base motions which consist of a constant angular velocity $\omega^{x}$ (or $\omega^{y}$ ) around the $O x$ axis (or the $O y$ axis) and a harmonic translation along the $O z$ axis given by $z_{O}=Z_{O} \sin \Omega^{z} t$. The resulting linear second-order differential equations of the dynamic system include constant terms and are solved by analytical methods in order to display the major 
basic phenomena occurring in rotordynamics with base motions. The equations of motion (31) are rewritten in equation (32) due to the rotation $\omega^{x}$ and equation (34) due to the rotation $\omega^{y}$

$$
\begin{aligned}
& \mathbf{M}_{d, s h} \ddot{\boldsymbol{\delta}}_{r}+\mathbf{C}_{d, s h}^{g} \Omega \dot{\boldsymbol{\delta}}_{r}+\left(\mathbf{K}_{s h}^{e}+\left(\mathbf{K}_{d, s h, b}^{r e, \omega^{r 2}}+\mathbf{K}_{s h, b}^{g s e, \omega^{r 2}}\right) \omega^{x 2}\right) \boldsymbol{\delta}_{r}=\mathbf{F}_{m u}^{c} \cos \Omega t+\mathbf{F}_{m u}^{s} \sin \Omega t \\
& +\mathbf{F}_{m u, b 1}^{c} \cos \Omega t+\mathbf{F}_{m u, b 1}^{s} \sin \Omega t+\mathbf{F}_{d, s h, b 1}^{c} \cos \Omega^{z} t+\mathbf{F}_{d, s h, b 1}^{s} \sin \Omega^{z} t+\mathbf{F}_{d, s h, b}^{0},
\end{aligned}
$$

where

$$
\begin{gathered}
\mathbf{F}_{m u}^{c}=\mathbf{V}_{m u}^{c} \Omega^{2} \quad ; \quad \mathbf{F}_{m u}^{s}=\mathbf{V}_{m u}^{s} \Omega^{2} ; \quad \mathbf{F}_{m u, b 1}^{c}=\mathbf{V}_{m u, b}^{\omega^{x 2}, c} \omega^{x 2} ; \quad \mathbf{F}_{m u, b 1}^{s}=\mathbf{V}_{m u, b}^{\omega^{x 2}, s} \omega^{x 2}, \\
\mathbf{F}_{d, s h, b 1}^{c}=\mathbf{0} ; \quad \mathbf{F}_{d, s h, b 1}^{s}=\mathbf{V}_{d, s h, b}^{w} Z_{O}\left(\Omega^{z 2}+\omega^{x 2}\right) ; \quad \mathbf{F}_{d, s h, b}^{0}=-\mathbf{V}_{d, s h, b}^{y \psi} \Omega \omega^{x} . \\
\mathbf{M}_{d, s h} \ddot{\boldsymbol{\delta}}_{r}+\left(\mathbf{C}_{d, s h}^{g} \Omega+\mathbf{C}_{d, s h, b}^{r e, \omega^{y}} \omega^{y}\right) \dot{\boldsymbol{\delta}}_{r}+\left(\mathbf{K}_{s h}^{e}+\mathbf{K}_{d, s h, b}^{r e, \Omega \omega^{y}} \Omega \omega^{y}+\mathbf{K}_{d, s h, b}^{r e, \omega^{y 2}} \omega^{y 2}\right) \boldsymbol{\delta}_{r}=\mathbf{F}_{m u}^{c} \cos \Omega t+\mathbf{F}_{m u}^{s} \sin \Omega t \\
+\mathbf{F}_{m u, b 2}^{c} \cos \Omega t+\mathbf{F}_{m u, b 2}^{s} \sin \Omega t+\mathbf{F}_{d, s h, b 2}^{c} \cos \Omega^{z} t+\mathbf{F}_{d, s h, b 2}^{s} \sin \Omega^{z} t,
\end{gathered}
$$

where

$$
\begin{aligned}
& \mathbf{F}_{m u, b 2}^{c}=\mathbf{V}_{m u, b}^{\Omega \omega^{y}, c} \Omega \omega^{y}+\mathbf{V}_{m u, b}^{\omega^{y 2}, c} \omega^{y 2} \quad ; \quad \mathbf{F}_{m u, b 2}^{s}=\mathbf{V}_{m u, b}^{\Omega \omega^{y}, s} \Omega \omega^{y}+\mathbf{V}_{m u, b}^{\omega^{y 2}, s} \omega^{y 2}, \\
& \mathbf{F}_{d, s h, b 2}^{c}=-2 \mathbf{V}_{d, s h, b}^{u} Z_{O} \Omega^{z} \omega^{y} \quad ; \quad \mathbf{F}_{d, s h, b 2}^{s}=\mathbf{V}_{d, s h, b}^{w} Z_{O}\left(\Omega^{z 2}+\omega^{y 2}\right) .
\end{aligned}
$$

In equations (33) and (35), $\mathbf{F}_{m u}^{c}, \mathbf{F}_{m u}^{s}, \mathbf{F}_{m u, b}^{c}, \mathbf{F}_{m u, b}^{s}, \mathbf{F}_{d, s h, b}^{c}, \mathbf{F}_{d, s h, b}^{s}$ are the amplitudes of sinusoidal force vectors, while $\mathbf{F}_{d, s h, b}^{0}$ is a constant force vector.

As $\omega^{x}$ and $\omega^{z}$ have similar influences on rotordynamics, only $\omega^{x}$ is considered in this study.

\subsection{Asymmetric rotor}

The disk is assumed to be asymmetric, while the shaft is symmetric. The rigid base and thereby the rotor are subjected to the same excitations as indicated in Section 5.2. However, the left-hand side of the linear equations of motion contains periodic parametric coefficients and their right-hand side contains load vectors multiplied by trigonometric functions due to the geometric asymmetry of the rotor. Thus the equations containing the Rayleigh damping are solved by a numerical time integration method. The equations of motion (31) are rewritten in equation (36) due to the motion $\omega^{x}$ and equation (38) due to the motion $\omega^{y}$

$$
\begin{aligned}
& \left(\mathbf{M}_{d, s h}+\mathbf{M}_{d, s h}^{c} \cos 2 \Omega t+\mathbf{M}_{d, s h}^{s} \sin 2 \Omega t\right) \ddot{\boldsymbol{\delta}}_{r}+\left(\mathbf{C}_{d, s h}^{i d}+\mathbf{C}_{d, s h}^{g} \Omega+\mathbf{C}_{d, s h}^{g, c} \Omega \cos 2 \Omega t+\mathbf{C}_{d, s h}^{g, s} \Omega \sin 2 \Omega t\right) \dot{\boldsymbol{\delta}}_{r} \\
& +\left(\mathbf{K}_{s h}^{e}+\left(\mathbf{K}_{d, s h, b}^{r e, \omega^{r 2}}+\mathbf{K}_{s h, b}^{g s e, \omega^{r 2}}\right) \omega^{x 2}+\mathbf{K}_{d, s h, b}^{r e, \omega^{r 2}, c} \omega^{x 2} \cos 2 \Omega t+\mathbf{K}_{d, s h, b}^{r e, \omega^{r 2, s}} \omega^{x 2} \sin 2 \Omega t\right) \boldsymbol{\delta}_{r}=\mathbf{F}_{m u}^{c} \cos \Omega t+\mathbf{F}_{m u}^{s} \sin \Omega t \\
& +\mathbf{F}_{m u, b 1}^{c} \cos \Omega t+\mathbf{F}_{m u, b 1}^{s} \sin \Omega t+\mathbf{F}_{d, s h, b 1}^{c} \cos \Omega^{z} t+\mathbf{F}_{d, s h, b 1}^{s} \sin \Omega^{z} t+\mathbf{F}_{d, s h, b}^{0}+\mathbf{F}_{d, s h, b 3}^{c} \cos 2 \Omega t+\mathbf{F}_{d, s h, b 3}^{s} \sin 2 \Omega t,
\end{aligned}
$$

where

$$
\begin{gathered}
\mathbf{F}_{d, s h, b 3}^{c}=2 \mathbf{V}_{d, s h, b}^{\psi, d i} \Omega \omega^{x} ; \mathbf{F}_{d, s h, b 3}^{s}=2 \mathbf{V}_{d, s h, b}^{\theta, d i} \Omega \omega^{x} . \\
\left(\mathbf{M}_{d, s h}+\mathbf{M}_{d, s h}^{c} \cos 2 \Omega t+\mathbf{M}_{d, s h}^{s} \sin 2 \Omega t\right) \ddot{\boldsymbol{\delta}}_{r}+\left(\mathbf{C}_{d, s h}^{i d}+\mathbf{C}_{d, s h}^{g} \Omega+\mathbf{C}_{d, s h}^{g, c} \Omega \cos 2 \Omega t+\mathbf{C}_{d, s h}^{g, s} \Omega \sin 2 \Omega t\right. \\
\left.+\mathbf{C}_{d, s h, b}^{r e, \omega^{y}} \omega^{y}\right) \dot{\boldsymbol{\delta}}_{r}+\left(\mathbf{K}_{s h}^{e}+\mathbf{K}_{d, s h, b}^{r e, \Omega \omega^{y}} \Omega \omega^{y}+\mathbf{K}_{d, s h, b}^{r e, \omega^{y 2}} \omega^{y 2}+\left(\mathbf{K}_{d, s h, b}^{r e, \Omega \omega^{y}, c} \Omega \omega^{y}+\mathbf{K}_{d, s h, b}^{r e, \omega^{y 2}, c} \omega^{y 2}\right) \cos 2 \Omega t\right. \\
\left.+\left(\mathbf{K}_{d, s h, b}^{r e, \Omega \omega^{y}, s} \Omega \omega^{y}+\mathbf{K}_{d, s h, b}^{r e, \omega^{y 2}, s} \omega^{y 2}\right) \sin 2 \Omega t\right) \boldsymbol{\delta}_{r}=\mathbf{F}_{m u}^{c} \cos \Omega t+\mathbf{F}_{m u}^{s} \sin \Omega t+\mathbf{F}_{m u, b 2}^{c} \cos \Omega t+\mathbf{F}_{m u, b 2}^{s} \sin \Omega t \\
+\mathbf{F}_{d, s h, b 2}^{c} \cos \Omega^{z} t+\mathbf{F}_{d, s h, b 2}^{s} \sin \Omega^{z} t .
\end{gathered}
$$

The elementary vectors and matrices involved in equations (32) to (38) are presented in Appendix A.

\section{Dynamic analysis}

Deterministic rotational and translational excitations of the rotor base have been stated in the previous section for two rotor configurations. The first one is a symmetric rotor and treated through analytical methods to seek its natural frequencies and to compute its steady-state responses and its orbits. The second one is an asymmetric rotor and examined through the Floquet theory to evaluate its dynamic stability as well as the Newmark method to predict its steady-state orbits in the time domain.

\subsection{Equations with constant coefficients}

In this case, the system of linear differential equations (31) contains constant matrices (according to equations (32) and (34)). It is transformed into a first-order differential equation by introducing the state-space vector $\mathbf{D}_{r}$, i.e.,

where

$$
\dot{\mathbf{D}}_{r}=\mathbf{A}_{r} \mathbf{D}_{r}+\mathbf{B}_{r},
$$




$$
\mathbf{D}_{r}=\left\{\begin{array}{l}
\boldsymbol{\delta}_{r} \\
\dot{\boldsymbol{\delta}}_{r}
\end{array}\right\} \quad ; \quad \mathbf{A}_{r}=\left[\begin{array}{cc}
\mathbf{0} & \mathbf{I} \\
-\mathbf{M}_{r}^{-1} \mathbf{K}_{r} & -\mathbf{M}_{r}^{-1} \mathbf{C}_{r}
\end{array}\right] \quad ; \quad \mathbf{B}_{r}=\left\{\begin{array}{c}
\mathbf{0} \\
\mathbf{M}_{r}^{-1} \mathbf{F}_{r}(t)
\end{array}\right\} .
$$

\subsubsection{Natural frequencies}

The rotor is studied in free motion and the homogeneous system is thus considered, i.e., equation (39) with no external forces. The eigenvalues of the matrix $\mathbf{A}_{r}$ are calculated for seeking the natural frequencies $f_{r}$ of the rotor. Therefore the Campbell diagram can be plotted to represent the frequencies $f_{r}$ as a function of the speed of rotation $\Omega$ of the rotor.

\subsubsection{Steady-state responses and orbits}

Since the steady-state responses are sought, only the particular solution of the equations of motion is considered. Due to the form of the external force vectors in the right-hand side, this solution adopts the following

$$
\boldsymbol{\delta}_{r}=\boldsymbol{\delta}_{m u}^{c} \cos (\Omega t)+\boldsymbol{\delta}_{m u}^{s} \sin (\Omega t)+\boldsymbol{\delta}_{b}^{c} \cos \left(\Omega^{z} t\right)+\boldsymbol{\delta}_{b}^{s} \sin \left(\Omega^{z} t\right)+\boldsymbol{\delta}_{b}^{0},
$$

$\boldsymbol{\delta}_{m u}^{c}, \boldsymbol{\delta}_{m u}^{s}, \boldsymbol{\delta}_{b}^{c}, \boldsymbol{\delta}_{b}^{s}$ and $\boldsymbol{\delta}_{b}^{0}$ are unknown displacement amplitude vectors. Using $\boldsymbol{\delta}_{r}$, the velocity as well as acceleration vectors $\dot{\boldsymbol{\delta}}_{r}, \ddot{\boldsymbol{\delta}}_{r}$ are obtained. Substituting them into equation (39) yields the steady-state solutions

$$
\begin{gathered}
{\left[\begin{array}{cc}
\mathbf{K}_{r}-\mathbf{M}_{r} \Omega^{2} & \mathbf{C}_{r} \Omega \\
-\mathbf{C}_{r} \Omega & \mathbf{K}_{r}-\mathbf{M}_{r} \Omega^{2}
\end{array}\right]\left\{\begin{array}{l}
\boldsymbol{\delta}_{m u}^{c} \\
\boldsymbol{\delta}_{m u}^{s}
\end{array}\right\}=\left\{\begin{array}{l}
\mathbf{F}_{m u}^{c}+\mathbf{F}_{m u, b}^{c} \\
\mathbf{F}_{m u}^{s}+\mathbf{F}_{m u, b}^{s}
\end{array}\right\},} \\
{\left[\begin{array}{cc}
\mathbf{K}_{r}-\mathbf{M}_{r} \Omega^{z 2} & \mathbf{C}_{r} \Omega^{z} \\
-\mathbf{C}_{r} \Omega^{z} & \mathbf{K}_{r}-\mathbf{M}_{r} \Omega^{z 2}
\end{array}\right]\left\{\begin{array}{c}
\boldsymbol{\delta}_{b}^{c} \\
\boldsymbol{\delta}_{b}^{s}
\end{array}\right\}=\left\{\begin{array}{l}
\mathbf{F}_{d, s h, b}^{c} \\
\mathbf{F}_{d, s h, b}^{s}
\end{array}\right\},} \\
\boldsymbol{\delta}_{b}^{0}=\mathbf{K}_{r}^{-1} \mathbf{F}_{d, s h, b}^{0} .
\end{gathered}
$$

The constant vector $\delta_{b}^{0}$ is nil in the case of the angular velocity $\omega^{y}$, i.e., of the base motion around the $O y$ axial axis (see equation 34) but not in the case of $\omega^{x}$, i.e., of the base motion around the $O x$ transverse axis (see equation 32). It is concluded that equation (42) is used to investigate the mass unbalance response of the rotor, equation (43) is employed to observe the frequency response of the rotor to the translational base motion and equation (41) helps to illustrate the steady-state orbits described by the points situated along the shaft elastic line.

\subsection{Equations with time-varying coefficients}

In this case, the system of linear differential equations (31) has periodic parametric coefficients (according to equations (36) and (38)) of period $\tau=\pi / \Omega$.

\subsubsection{Dynamic stability}

The stability of the periodic solution is predicted by employing the Floquet theory. In other words, by determining the so-called "Floquet transition matrix" (or monodromy matrix) during one period of the parametric excitation and by finding its eigenvalues, the zones of instability are identified, see Evan-Iwanowski (1976), Dufour and Berlioz (1998) as well as Berlioz et al. (2000). First, the application of the Floquet theory requires a homogeneous differential system represented in the state-space form. Namely, the linear second-order differential equations (31) of the rotor become homogeneous and have to be transformed into a first-order system

$$
\dot{\mathbf{D}}_{r}=\mathbf{A}_{r} \mathbf{D}_{r} \text {. }
$$

Then, the system of equation (45) of the rotor is integrated over one period $\tau=\pi / \Omega$ using the Runge-Kutta time-step integration scheme with the following initial conditions at time $t=0$

$$
\mathbf{D}_{r}(0)=\langle 0,0, \ldots, 1, \ldots, 0\rangle^{T},
$$

where only the $i$-th component is not nil and equal to 1 . For each integration, the vector $\mathbf{D}_{r}(\tau)$ is obtained and represents the solution of equation (45) at the end of one period $\tau$ and the $i$-th column of the Floquet transition matrix $\boldsymbol{\varphi}_{r}(\tau)$. To provide the Floquet transition matrix at time $t=\tau, 2 n_{g}$ integrations are necessary where $n_{g}$ is the total number of degrees of freedom of equation (31). The eigenvalues or characteristic multipliers of the Floquet transition matrix $\boldsymbol{\varphi}_{r}(\tau)$ of the system (45) are calculated. Finally, the system (45) is unstable if the modulus of at least one of the characteristic multipliers is greater than 1 .

\subsubsection{Time history responses and orbits}

In the presence of time-varying parametric coefficients, the linear equations of motion (31) are solved using the Newmark time-step integration algorithm based on the average acceleration to predict the rotor orbits when the steady-state responses are reached, i.e., the integration algorithm requires the presence of the damping to overcome the transient regime. 


\section{Simulation results and discussion}

\subsection{Symmetric rotor}

\subsubsection{Configuration and data}

Figure 5 and Table 1 present the components and the characteristics of the symmetric on-board rotor. The origin $O$ of the reference frame $R$ coincides with the shaft end-point positioned at the center of the bearing \# 1 . The symmetric shaft is modeled with 12 identical two-node Timoshenko beam FEs. The symmetric disk is located at node 5. The rigid short bearings \# 1 and \# 2 generate a pinned-pinned rotor and are located at nodes 1 and 13. Thus the corresponding degrees of freedom are canceled. The mass unbalance is placed on the disk.

The investigated rotor runs at a constant speed of rotation $\Omega$ and is subjected to mass unbalance excitation as well as to combined constant rotation and harmonic translation of the rigid base: the constant rotation around the $O x$ axis (or the $O y$ axis) is given by $\omega^{x}$ (or $\omega^{y}$ ), while the harmonic translation along the $O z$ axis is expressed as $z_{O}=Z_{O} \sin \Omega^{z} t$. The steady-state dynamic analysis is performed by means of Campbell diagrams, mass unbalance responses, responses to the harmonic translational base excitations and rotor orbits. In addition, the equations of motion of the on-board rotor are parametrically affected (i.e., the left-hand side of equations (32) and (34)) by the base rotations, while the base translations have only an effect on the external force vector (i.e., on the right-hand side). Therefore the overall behavior is analyzed as a function of the base rotation.

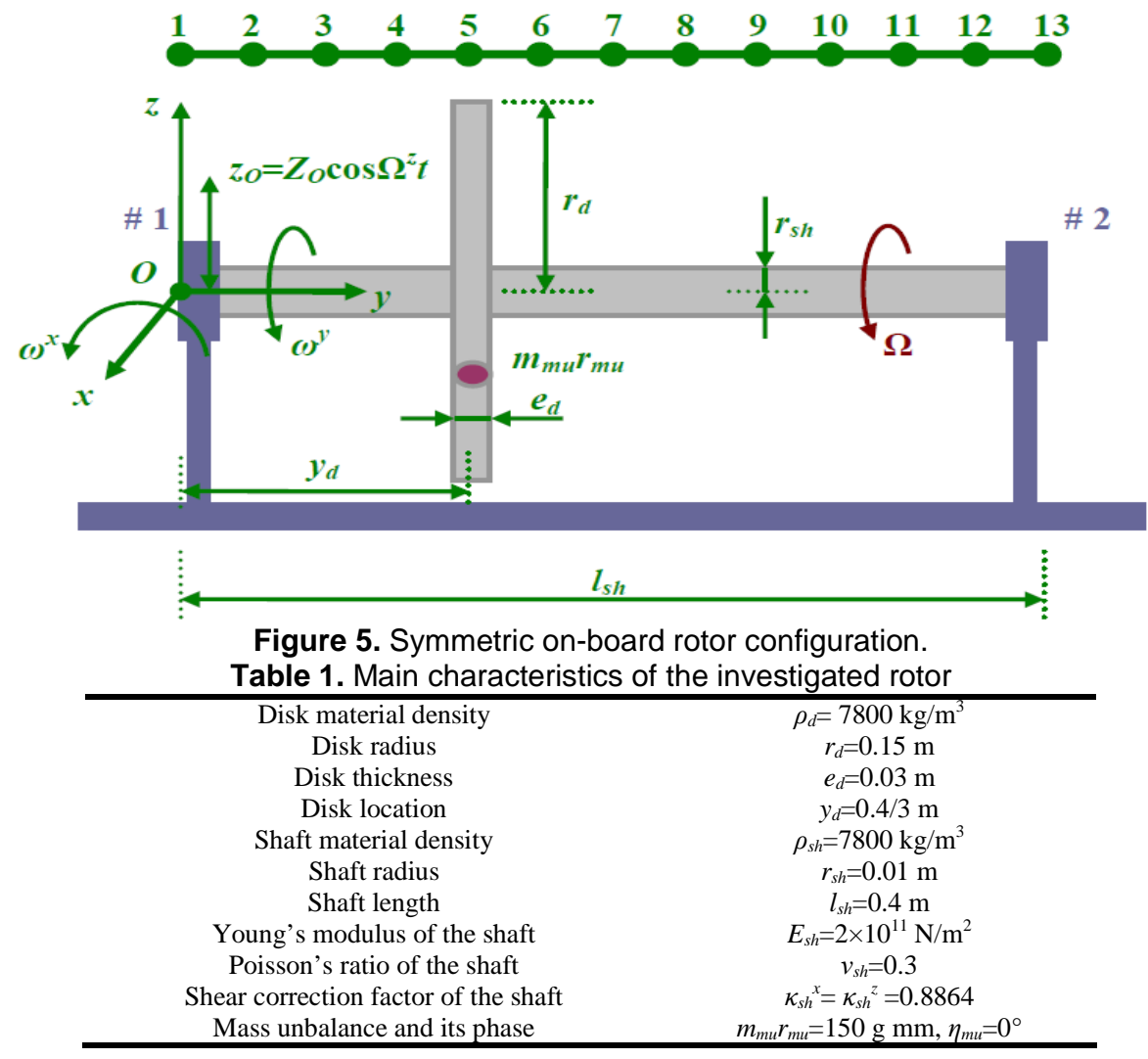

\subsubsection{Campbell diagrams}

In the case of a symmetric rotor, the linear equations of motion always display constant coefficients, see equations (32) and (34). It should be mentioned that a fixed base or a rotating base around the $O y$ axis keep the system isotropic, while a base rotation around the $O x$ axis makes it anisotropic. Moreover, the sign of the components of the damping and stiffness matrices depends on the direction of the base rotation around $O y$ but not on that of the base rotation around $O x$. In the case of a positive direction (counterclockwise) of the base rotation around $O y$ and any direction around $O x$, the symmetric stiffness matrices can lose their positive definiteness because of the presence of negative diagonal terms containing the angular velocity $\omega^{x}$ or $\omega^{y}$. Thus a possible dynamic instability of the rotor can occur. In the presented application, since the eigenvalues of the state-space system (39) with no external forces are purely imaginary quantities whatever the constant angular velocity of the base rotations in the zone of interest $\omega^{x}$ or $\omega^{y} \in[0 ; 10 \mathrm{~Hz}]$, the on-board rotor is stable for all the considered mode shapes included in its operating range $\Omega \in[0 ; 6000 \mathrm{rpm}]$. The natural frequencies $f_{r}$ being deduced from these eigenvalues depend on the speed of rotation $\Omega$ of the rotor and on the angular velocity of 
the base rotations either around $O x$ or $O y$. They are represented by the Campbell diagrams plotted in Figure 6 for the rotor subjected to the angular velocity either $\omega^{x}$ or $\omega^{y}$. When the rotor base is fixed $\left(\omega^{x}=\omega^{y}=0\right)$, the system is isotropic and therefore the dynamic behavior of the rotor is symmetric, i.e., the natural frequencies of each pair of backward and forward whirl modes are equal at rest $(\Omega=0)$. Even if the natural frequencies strongly do not change, the base rotation $\omega^{x}$ breaks the symmetry of the rotor behavior, see Figure 6(a). Since the corresponding stiffness matrices are symmetric with anisotropic terms and there is no damping matrix associated with $\omega^{x}$, the zoom shows that the natural frequencies of the first backward and forward whirl modes at rest are non-equal, this justifies that the behavior of the rotor is non-symmetric. The rotational effects due to $\omega^{x}$ decrease the natural frequencies, while the geometric stiffening effects associated with the centrifugal stressing relative to $\omega^{x}$ increase them. Unlike the previous case, the solicitation due to the base rotation around $O y$ does not bring any asymmetry to the dynamic behavior of the rotor (because the corresponding matrices are symmetric and skew-symmetric with isotropic terms) but shifts and changes the natural frequencies (due to additional terms in the damping and stiffness matrices), see Figure 6(c). The damping matrix associated with $\omega^{y}$ makes the pairs of backward and forward whirl modes at rest unequal and reversed compared to the case of fixed base and that of $\omega^{x}$. Increasing the speed of rotation $\Omega$ of the rotor decreases the backward whirl frequencies and increases the forward ones. Table 2 introduces comparisons between the first four natural frequencies $f_{r}$ of the rotor relative to the base rotations either around $O x$ or $O y$. At any angular velocities, $\omega^{y}$ has an influence greater than $\omega^{x}$ on the natural frequencies, i.e., the natural frequencies of the rotor corresponding to the base rotation around the $O y$ axis are more different when compared to those obtained when the base is fixed.

(a)

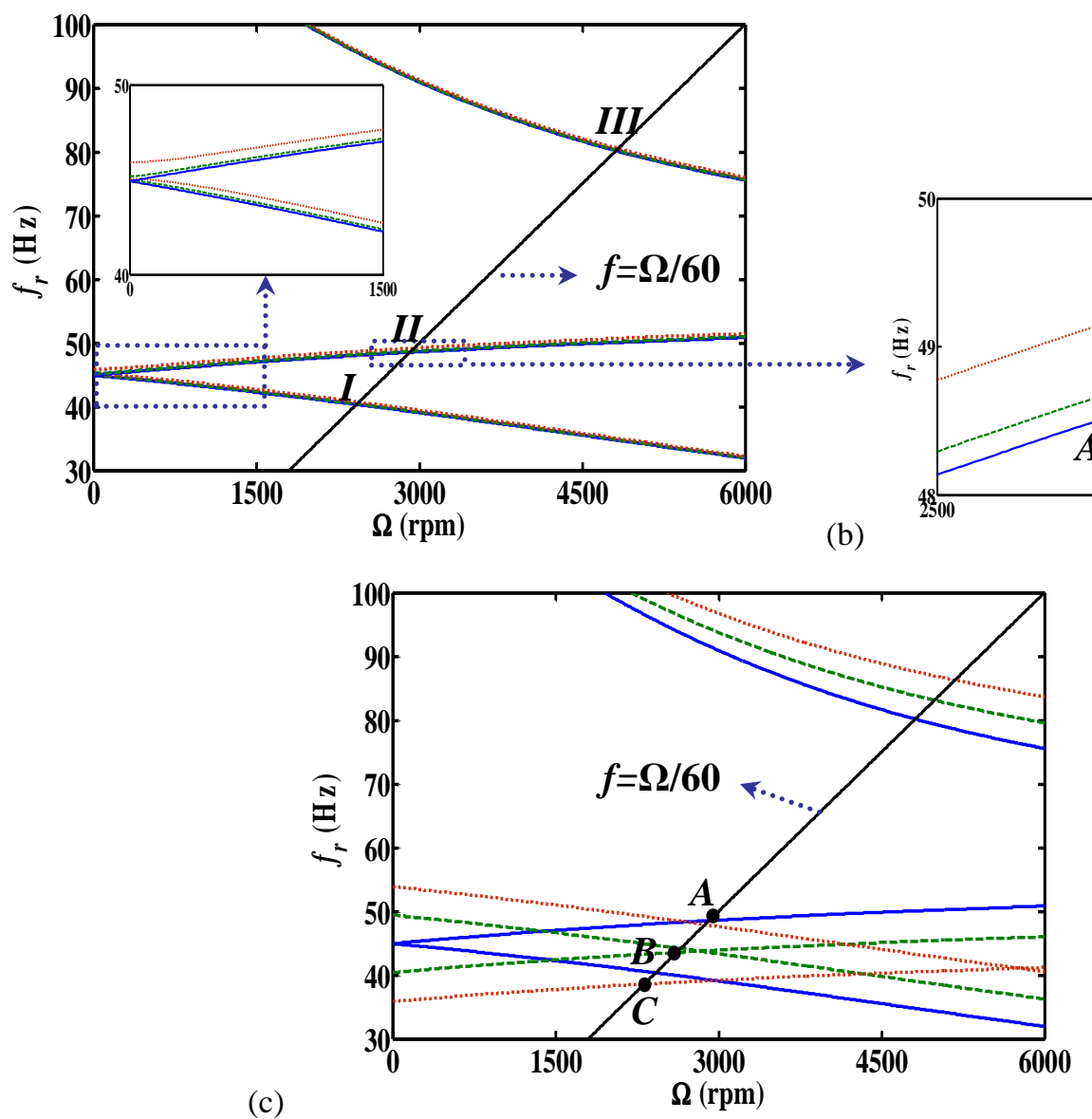

Figure 6. Campbell diagrams for the first three natural frequencies of the rotor subjected to the base angular velocity: (a) $\omega^{x}$, or (c) $\omega^{y}$, with the values: 0 (solid line), $5 \mathrm{~Hz}$ (dashed line), $10 \mathrm{~Hz}$ (dotted line), (b) Zoom on the intersection of the first forward whirl frequency with the speed of rotation of the rotor.

Table 2. Natural frequencies as a function of the speed of rotation of the rotor and the constant rotations of the base ( $B$ and $F$ stand for backward and forward whirl modes)

\begin{tabular}{ccccccc}
\hline \multirow{2}{*}{$\Omega$} & \multirow{2}{*}{$\begin{array}{c}* \\
\mathrm{rpm}\end{array}$} & $\mathrm{f}$ & \multicolumn{3}{c}{$\omega^{x}$} & \multicolumn{2}{c}{$\omega^{y}$} \\
& $\mathrm{~Hz}$ & base & \multicolumn{2}{c}{$\mathrm{Hz}$} & \multicolumn{2}{c}{$\mathrm{Hz}$} \\
\cline { 4 - 7 } & & & 5 & 10 & 5 & 10 \\
\hline \multirow{2}{*}{0} & $f_{1}^{B}$ & 44.93 & 44.96 & 45.04 & 49.42 & 53.88 \\
& $f_{1}^{F}$ & 44.93 & 45.19 & 45.93 & 40.42 & 35.88 \\
& $f_{2}{ }^{B}$ & 123.79 & 123.80 & 123.84 & 124.49 & 125.42 \\
& $f_{2}^{F}$ & 123.79 & 123.88 & 124.15 & 123.32 & 123.09 \\
\hline 1500 & $f_{1}^{B}$ & 42.28 & 42.40 & 42.73 & 46.65 & 50.99 \\
\hline
\end{tabular}




\begin{tabular}{ccccccc}
\hline \multirow{6}{*}{3000} & $f_{1}{ }^{F}$ & 47.02 & 47.17 & 47.66 & 42.40 & 37.76 \\
& $f_{2}{ }^{B}$ & 104.64 & 104.70 & 104.90 & 106.48 & 108.54 \\
& $f_{2}{ }^{F}$ & 148.73 & 148.77 & 148.90 & 149.40 & 150.27 \\
\hline \multirow{6}{*}{4500} & $f_{1}{ }^{B}$ & 39.07 & 39.18 & 39.48 & 43.36 & 47.63 \\
& $f_{1}{ }^{F}$ & 48.62 & 48.78 & 49.27 & 43.92 & 39.20 \\
& $f_{2}{ }^{B}$ & 90.93 & 91.02 & 91.27 & 93.76 & 96.75 \\
& $f_{2}{ }^{F}$ & 179.05 & 179.08 & 179.18 & 180.69 & 182.49 \\
\hline \multirow{3}{*}{6000} & $f_{1}{ }^{B}$ & 35.53 & 35.62 & 35.86 & 39.79 & 44.04 \\
& $f_{1}{ }^{F}$ & 49.86 & 50.02 & 50.51 & 45.10 & 40.32 \\
& $f_{2}{ }^{B}$ & 81.66 & 81.76 & 82.07 & 85.22 & 88.89 \\
& $f_{2}{ }^{F}$ & 213.80 & 213.83 & 213.92 & 216.20 & 218.73 \\
\hline & $f_{1}{ }^{B}$ & 31.96 & 32.03 & 32.23 & 36.26 & 40.56 \\
& $f_{1}{ }^{F}$ & 50.82 & 50.99 & 51.48 & 46.02 & 41.20 \\
& $f_{2}{ }^{B}$ & 75.55 & 75.67 & 76.03 & 79.60 & 83.72 \\
& $f_{2}{ }^{F}$ & 251.95 & 251.97 & 252.05 & 254.91 & 257.97 \\
\hline
\end{tabular}

\subsubsection{Responses to the mass unbalance or to the translational base excitation}

The rotor response due to the mass unbalance effect can be related to the constant rotational base motions, see equations (32) to (35). Figure 7 presents the mass unbalance responses at the middle of the shaft (i.e., node 7) of the rotor excited by the angular velocity either $\omega^{x}$ or $\omega^{y}$. In the operating range $\Omega \in[0 ; 6000 \mathrm{rpm}]$, the rotor exhibits one principal critical speed of rotation (one peak $I I$ ) when the base is fixed. It exhibits only one peak in the presence of the base rotation $\omega^{y}$ (due to the isotropic system), see Figure 7(b), but three peaks $(I, I I, I I I)$ in the presence of $\omega^{x}$ (due to the anisotropic system), see Figure 7(a). These peaks correspond to the intersection of the straight line $f=\Omega / 60$ ( $\Omega$ in rpm) with the natural frequencies of the rotor as shown in Figure 6, i.e., the intersection with the frequencies of the first and second backward whirl modes $(I, I I I)$ and of the first forward whirl mode (II ) for $\omega^{x}$ and the intersection with the frequencies of the first forward whirl mode for $\omega^{y}$. The responses confirm that the rotor keeps a symmetric dynamic behavior in the case of the base rotation around $O y$ but has a non-symmetric dynamic behavior in the case of the base rotation around $O x$ as stated in Section 7.1.2 and presented in Figure 6. For increasing values of $\omega^{x}$, the principal critical speed (II ) increases slightly as shown by the points $(A, B, C)$ in Figure $6(\mathrm{~b})$ and the amplitudes of the additional peaks $(I, I I I)$ become larger and larger. As shown by the points $(A, B, C)$ in Figure 6(c), the critical speed position is shifted down when the angular velocity $\omega^{y}$ increases because the backward and forward whirl modes at rest $(\Omega=0)$ are reversed. It is also observed that even if the rotor is at rest, the amplitudes of the mass unbalance response are not nil due to the presence of the base rotation either around $O x$ or $O y$. In addition, these amplitudes become larger when the angular velocity of the base rotation increases, see Table 3 . At the highest speeds of rotation of the rotor, the corresponding response amplitudes reach the limiting values which are roughly equal to $12.431 \times 10^{-6} \mathrm{~m}$ for $\omega^{x}$ and $11.512 \times 10^{-6} \mathrm{~m}$ for $\omega^{y}$.

Under combined motions of the base, the rotor response due to the harmonic translational base motions depends on the constant rotations of the base, see equations (32) to (35). Figure 8 presents the evolution of the frequency responses at the middle of the shaft (i.e., node 7) of the rotor running at a speed of rotation $\Omega=1500 \mathrm{rpm}(=25 \mathrm{~Hz})$ and excited by a harmonic base translation of amplitude $Z_{O}=1 \times 10^{-6} \mathrm{~m}$ in the $\mathrm{Oz}$ direction. The frequency of the base translation is contained in the range of interest $\Omega^{z} \in[0 ; 200 \mathrm{~Hz}]$. In the presence of the angular velocity $\omega^{x}$ or $\omega^{y}$, the responses always exhibit four critical frequencies (four peaks) corresponding to the natural frequencies of the rotor at $\Omega=1500 \mathrm{rpm}$, see also the Campbell diagrams plotted in

Figure 6 and presented in Table 2. In the case of increasing values of $\omega^{y}$, the peak relative to the third natural frequency and produced by the harmonic base translation along the $O z$ axis becomes smaller and smaller, see Figure 8(b). This is because equation (34) presents a negative cosine term containing the frequency $\Omega^{z}$ of the base translation. Lastly, it is noted that the operating zones of the frequency responses along the $\Omega^{z}$ axis do not change mostly for the angular velocity $\omega^{x}$, while they can change significantly for $\omega^{y}$. 

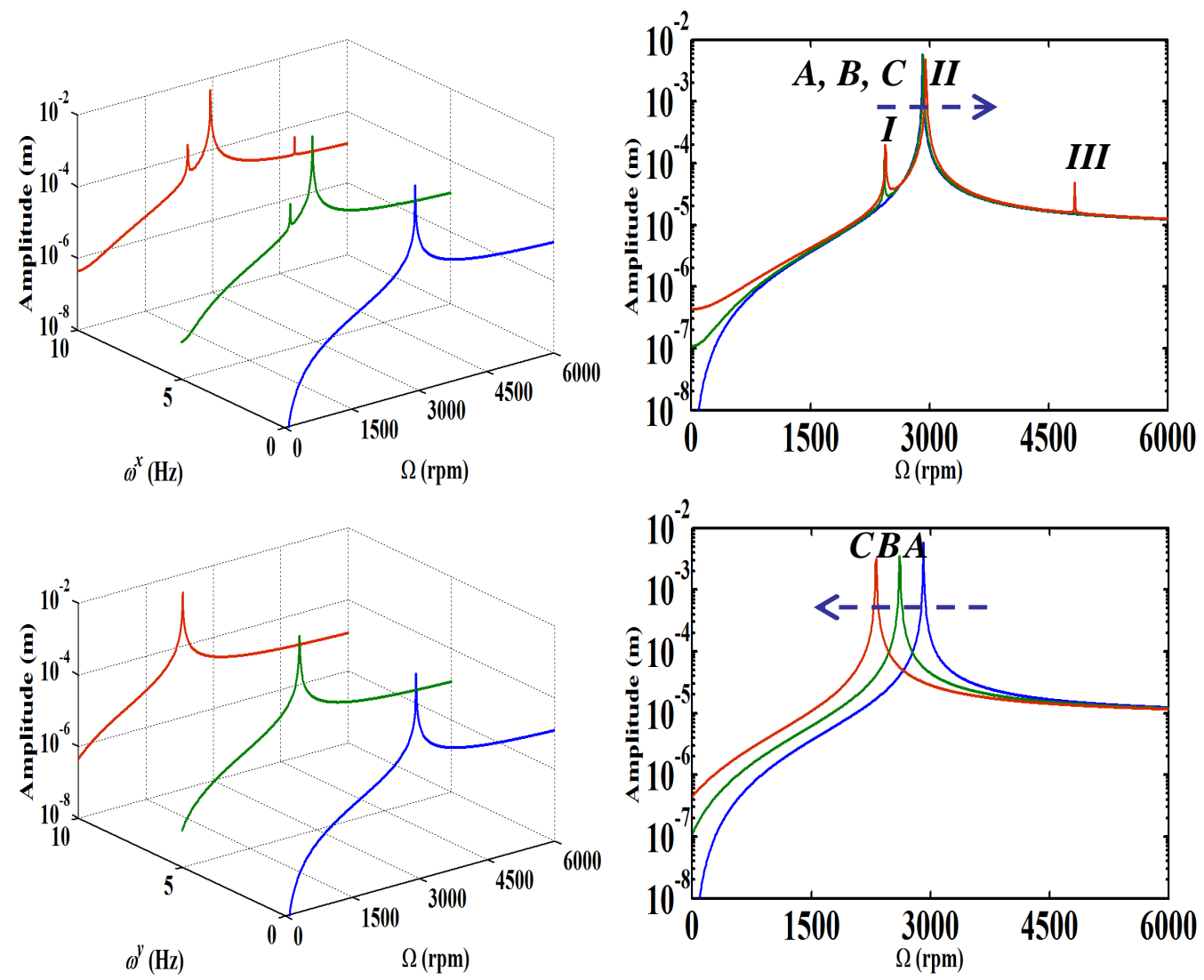

Figure 7. Mass unbalance responses of the rotor in the case of the constant rotation of the base around:

(a) the Ox axis, or (b) the Oy axis.

Table 3. Amplitudes of the mass unbalance responses of the rotor at rest for the angular velocity either $\omega^{x}$ or $\omega^{y}$

\begin{tabular}{cc|cc}
\hline$\omega^{x}$ & Amplitude & $\omega^{y}$ & Amplitude \\
$\mathrm{Hz}$ & $\mathrm{m}$ & $\mathrm{Hz}$ & $\mathrm{m}$ \\
\hline 5 & $0.107 \times 10^{-6}$ & 5 & $0.109 \times 10^{-6}$ \\
10 & $0.428 \times 10^{-6}$ & 10 & $0.449 \times 10^{-6}$ \\
\hline
\end{tabular}

\subsubsection{Influence of the base excitations on the orbits}

In this section, the orbits of the symmetric rotor subjected successively to a translation, a rotation and combined rotation and translation of the base are calculated with the finite element (FE) method. For validation purposes, a comparison is performed with results obtained by the Rayleigh-Ritz (RR) method which is based on the work of Duchemin (2003) and which has been extended in the present paper to account for a pure rotation and combined motions of the rotor base. The FE model is based on 48 degrees of freedom, while the RR method uses only one classical trigonometric function as a displacement function, i.e., two generalized coordinates $q_{1}(t)$ and $q_{2}(t)$

$$
u=\sin \left(\frac{\pi y}{l_{s h}}\right) q_{1} \quad ; \quad w=\sin \left(\frac{\pi y}{l_{s h}}\right) q_{2} .
$$

\subsubsection{Pure translation of the base and validation against the Rayleigh-Ritz method}

The orbits presented in Figure 9 are produced at the middle of the rotor presented in Section 7.1.1 and subjected to mass unbalance $\left(m_{m u} r_{m u}=15 \mathrm{~g} \mathrm{~mm}\right.$ and $\left.\eta_{m u}=0^{\circ}\right)$ and to harmonic base translation with $\left(Z_{O}=0.1 \mathrm{~m}\right.$ and $\Omega^{z}=2 \mathrm{~Hz}$ ) for a constant speed of rotation $\Omega=20000 \mathrm{rpm}$. Figure 9(a) compares the orbits obtained with the RR approaches and no deviation can be observable. Figure 9(b) presents the orbit predicted with the FE model. It is qualitatively similar to that of Figure 9(a) and exhibits very small magnitude deviations: the $x$ magnitude deviation is $\varepsilon_{u}=4.23 \%$ and the $z$ magnitude deviation is $\varepsilon_{w}=-0.58 \%$. In conclusion, the RR and FE models developed in this paper are validated regarding the RR model developed by Duchemin (2003). 

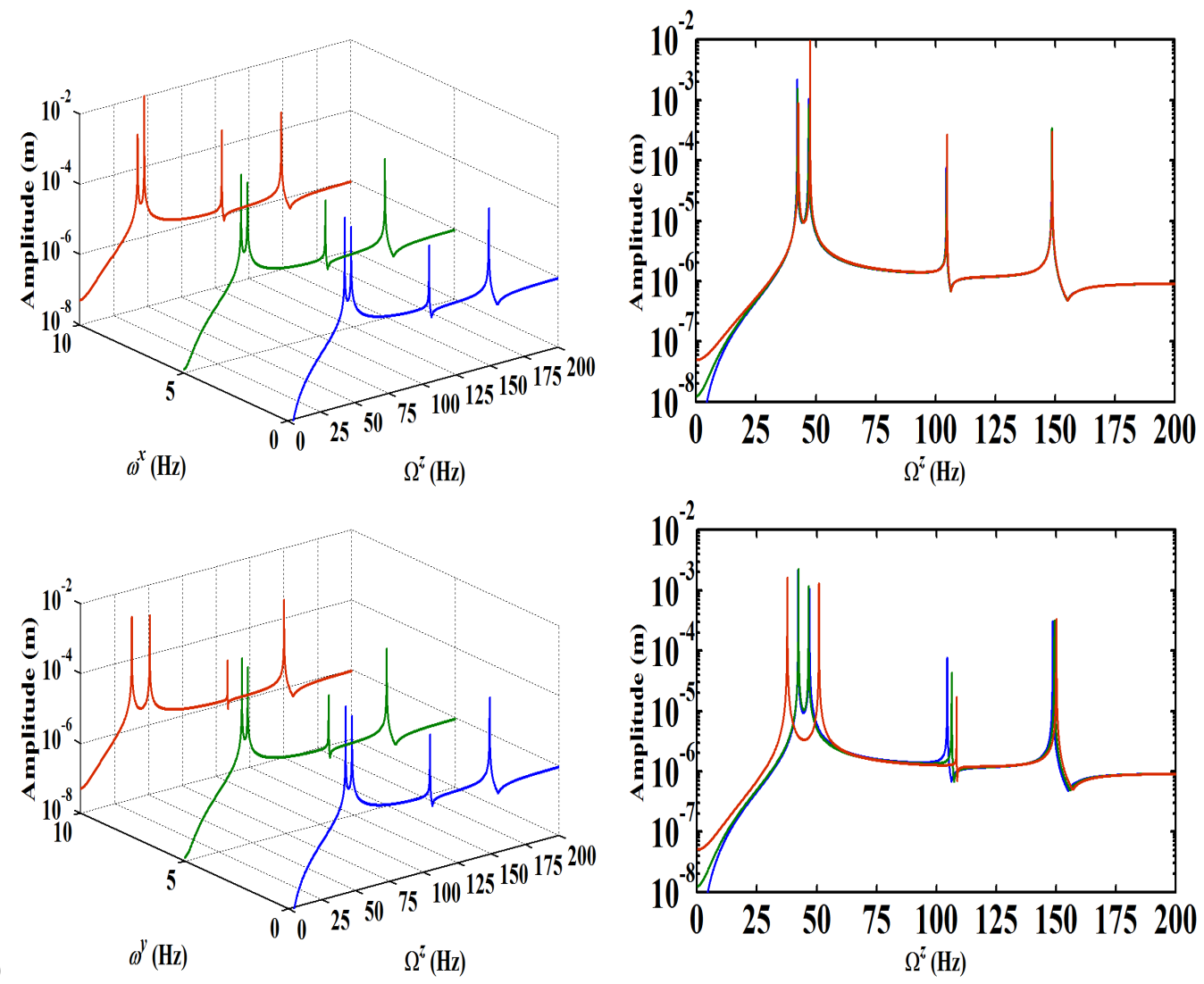

Figure 8. Frequency responses of the rotor to the sinusoidal base translation for a speed of rotation $\Omega=1500 \mathrm{rpm}$ and the constant rotation of the base around: (a) the Ox axis, or (b) the Oy axis.
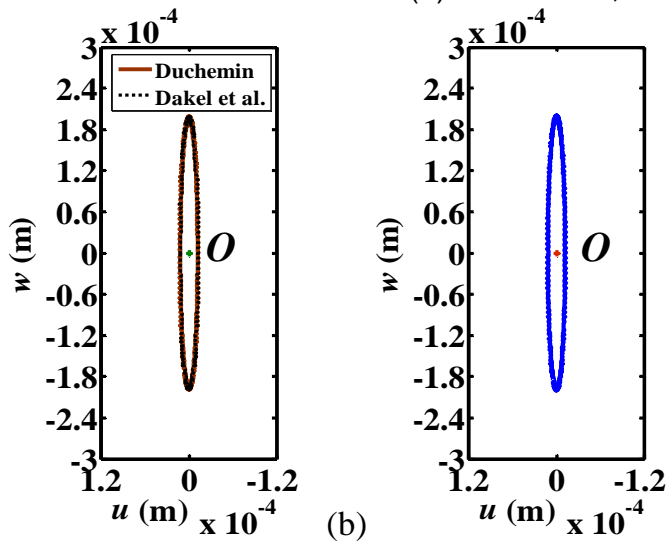

Figure 9. Orbits at the middle of the rotor running at $\Omega=20000 \mathrm{rpm}$ and subjected to a harmonic base translation with $Z_{O}=0.1 \mathrm{~m}$ and $\Omega^{z}=2 \mathrm{~Hz}$ using: (a) RR method, (b) FE method.

\subsubsection{Pure rotation $\omega^{x}$ (or $\left.\omega^{y}\right)$ of the base}

Figure 10(a) displays the classical orbit at the middle of the rotor (node 7) due to the mass unbalance for a constant speed of rotation $\Omega=1500 \mathrm{rpm}$ ( $=25 \mathrm{~Hz}=$ the mass unbalance frequency) and a fixed base. Since the matrices of the rotor system are symmetric and skew-symmetric with isotropic diagonal and cross-coupling components, the dynamic behavior is symmetric and the orbit is circular and periodic of period equal to the inverse of the mass unbalance frequency $\Omega$. The orbit center coincides with the point $O$ (bearing center) because the bearings are rigid and the motion amplitude of the rotor in both $O x$ and $O z$ directions is $3.628 \times 10^{-6}$ $\mathrm{m}$ at its middle. Figures $10(\mathrm{~b})$ and 10(c) compare the steady-state mass unbalance orbits in the case of a pure rotation of the base either around $O x$ or $O y$, see the solution in equation (41).

For the base angular velocity $\omega^{x}=5 \mathrm{~Hz}$, the corresponding trajectory illustrated by the orbits oscillates about a static position $C_{s p}$ due to the constant Coriolis force vector $\mathbf{F}_{d, s h, b}^{0}=-\mathbf{V}_{d, s h, b}^{y \psi} \Omega \omega^{x}$ acting in the $O x$ direction, see equation (32). The displacement yielding the static position $C_{s p}$ is calculated using equation (44) and its coordinate along $O x$ is approximately equal to $3.2 \times 10^{-3} \mathrm{~m}$. The coordinate of the static position displacement is very large compared to the orbit magnitude (dynamic displacement amplitude of the rotor) and thus forces the plot to adopt the point $C_{s p}$ as an origin. In addition, the periodic orbit is an ellipse due to the nonsymmetric dynamic behavior of the rotor and its orthogonal axes defining the $x$ and $z$ amplitudes of the 
flexural vibrations at the middle of the shaft are $3.554 \times 10^{-6} \mathrm{~m}$ and $3.784 \times 10^{-6} \mathrm{~m}$ respectively, i.e., they are slightly different.

For the base angular velocity $\omega^{y}=5 \mathrm{~Hz}$, there is no such a static position and the orbit center remains at the bearing center $O$. The periodic orbit keeps a circular shape because the symmetric dynamic behavior of the rotor is never broken, while its radius representing the $x$ and $z$ vibration amplitudes becomes greater than that obtained for a fixed base because the mass unbalance excitation increases in a way more important than the stiffness matrix of the rotor. Lastly, this radius is $6.183 \times 10^{-6} \mathrm{~m}$ at the middle of the shaft, i.e., the amplitude increases by a factor of about 1.7 in both transverse directions. The RR and FE mass unbalance orbits are qualitatively similar and show quantitatively very low $x$ and $z$ magnitude deviations: $\varepsilon_{u}=\varepsilon_{w}=-5.75 \%$.
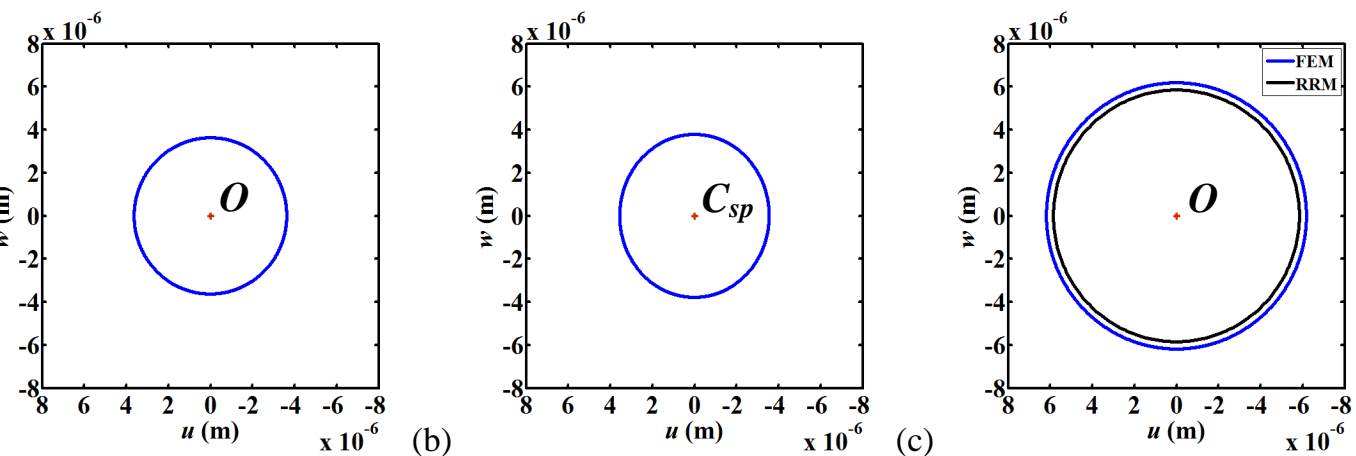

(a)
(b)

of base rotation: (a) $\omega^{x}=\omega^{y}=0$, i.e., fixed base, (b) $\omega^{x}=5 \mathrm{~Hz}, \omega^{y}=0$,

pm and subjected to three values

, (c) $\omega^{x}=0, \omega^{y}=5 \mathrm{~Hz}$.

\subsubsection{Combination of translation $z_{O}=Z_{O} \sin \Omega^{z} t$ and rotation $\omega^{x}\left(\right.$ or $\left.\omega^{y}\right)$ of the base}

Because the differential equations describing the rotor motion are linear (see equations (32) and (34)), the rotor orbits contain the response to the mass unbalance excitation superimposed with the response to the rotational and translational base motions. Thus the orbit characteristics (shape and size) depend on both excitations. In addition, the steady-state orbits are periodic because the external excitations due to both the mass unbalance and the base translation are sinusoidal and commensurable, see the solution in equation (41). The period of the orbits is equal to the inverse of the greatest common factor for the mass unbalance frequency $\Omega$ and the base translation frequency $\Omega^{z}$. Figures 11 to 13 give the orbits at the middle of the shaft (node 7) for a constant speed of rotation $\Omega=1500 \mathrm{rpm}(=25 \mathrm{~Hz})$ and different cases of combined rotational and translational motions of the rotor base.

In Figure 11, the base translation is kept constant $\left(Z_{O}=1 \times 10^{-6} \mathrm{~m}\right.$ and $\left.\Omega^{z}=200 \mathrm{~Hz}\right)$, while the base rotation varies $\left(\omega^{x}\right.$ or $\omega^{y}=5 \mathrm{~Hz}$ and $10 \mathrm{~Hz}$ ). Due to the sinusoidal base translation, complicated orbits appear instead of the classical circle or ellipse. As shown previously when the rotor is subjected to the angular velocity $\omega^{x}$, the rotor orbits oscillate about a static position $C_{s p}$. The coordinates along $O x$ of the displacements yielding the static position are calculated using equation (44) and increase with $\omega^{x}$. They are roughly $3.2 \times 10^{-3} \mathrm{~m}$ for $\omega^{x}=5 \mathrm{~Hz}$ and $6.3 \times 10^{-3} \mathrm{~m}$ for $\omega^{x}=10 \mathrm{~Hz}$. The plot adopts the static position $C_{s p}$ as an origin. The magnitudes

of the orbits remain almost the same for increasing values of $\omega^{x}$. When the rotor is excited by the angular velocity $\omega^{y}$, the plot adopts the bearing center $O$ as an origin. The magnitudes of the orbits increase for increasing values of $\omega^{y}$ because the mass unbalance excitation increases in a way more important than the stiffness matrix of the rotor. In Figure 12, the base rotation and the amplitude of the harmonic base translation are kept constant $\left(\omega^{x}\right.$ or $\omega^{y}=5 \mathrm{~Hz}$ and $Z_{O}=1 \times 10^{-6} \mathrm{~m}$ ), while the frequency of the base translation varies $\left(\Omega^{z}=100 \mathrm{~Hz}\right.$ and $120 \mathrm{~Hz}$ ). The frequency of the harmonic translational base excitation changes extremely the shape of the orbits, while it modifies slightly their magnitudes (when compared to those shown in Figure 11). As stated previously, the orbit period is equal to the inverse of the greatest common factor of the mass unbalance frequency $\Omega$ and the base translation frequency $\Omega^{z}$. Namely, one period of the above orbit corresponds to one period of the mass unbalance excitation and to four periods of the base translation for $\Omega^{z}=100 \mathrm{~Hz}$, while one period of the below orbit corresponds to five periods of the mass unbalance excitation and to 24 periods of the base translation in the case where $\Omega^{z}=120 \mathrm{~Hz}$. It should be noted that for several base translation frequencies different from the mass unbalance frequency, the orbit shapes become more complicated with respect to those obtained when the base is subjected to the pure rotation, see Figures 10(b) and 10(c). In Figure 13, the base rotation and the frequency of the base translation are kept constant ( $\omega^{x}$ or $\omega^{y}=5 \mathrm{~Hz}$ and $\Omega^{z}=200 \mathrm{~Hz}$ ), while the amplitude of the harmonic base translation varies $\left(Z_{O}=5 \times 10^{-6} \mathrm{~m}\right.$ and $\left.10 \times 10^{-6} \mathrm{~m}\right)$. Increasing the amplitudes makes the orbits much larger and more complicated especially when compared with the mass 
unbalance orbits for a base excited by a pure rotation, see Figure 10. The base translation has a large influence on the $z$ orbit magnitudes but a slight influence on the $x$ magnitudes.

The RR and FE orbits shown in Figure 14 are qualitatively the same but quantitatively different. The $x$ and $z$ magnitude deviations are $\varepsilon_{u}=-6.27 \%$ and $\varepsilon_{w}=0.04 \%$ for $\omega^{x}$ as well as $\varepsilon_{u}=-8.18 \%$ and $\varepsilon_{w}=-4.03 \%$ for $\omega^{y}$. The static position coordinate deviation is $\varepsilon_{s p}=-5.96 \%$.

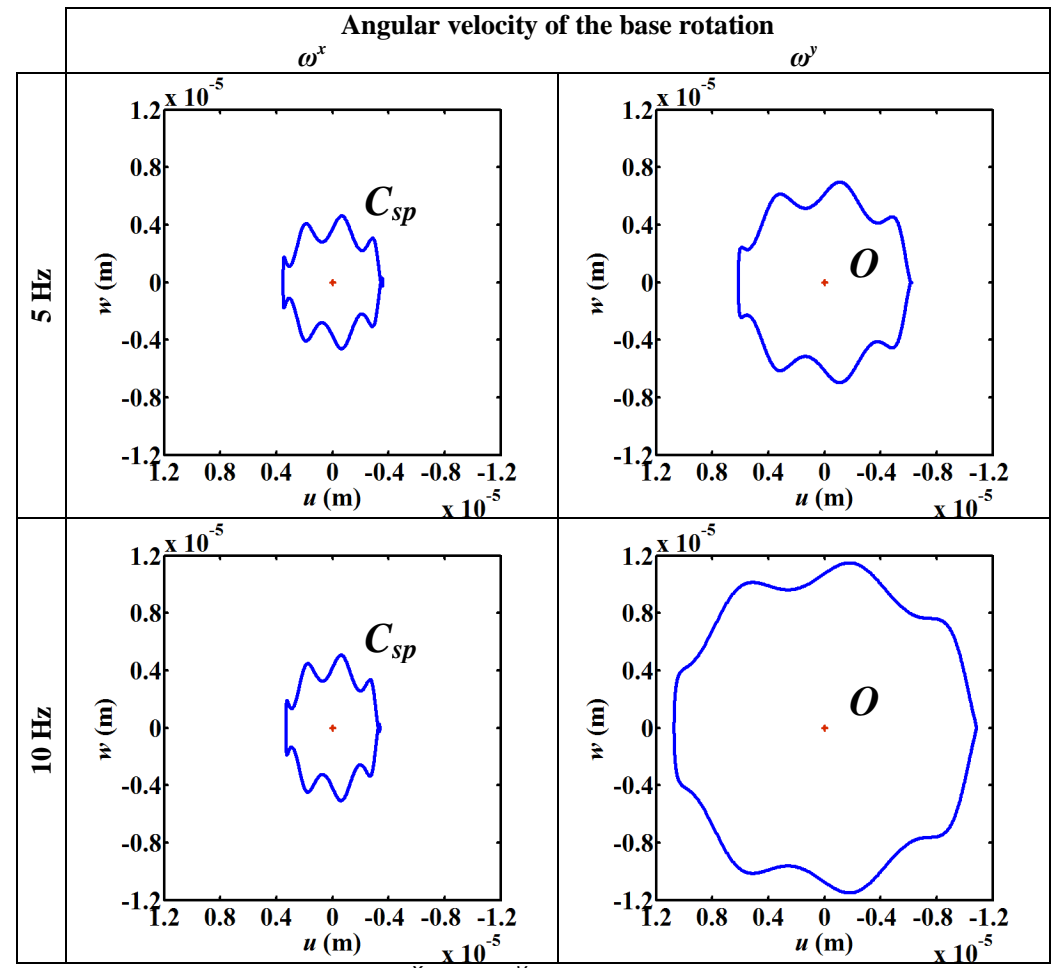

Figure 11. Influence of the angular velocity $\omega^{x}$ and $\omega^{y}$ of the base. Orbits at the middle of the rotor running at $\Omega=1500 \mathrm{rpm}(=25 \mathrm{~Hz})$ and subjected to a base translation: $z_{O}=Z_{O} \sin \left(\Omega^{z} t\right)$ with $Z_{O}=1 \times 10^{-6} \mathrm{~m}$ and $\Omega^{z}=200 \mathrm{~Hz}$.

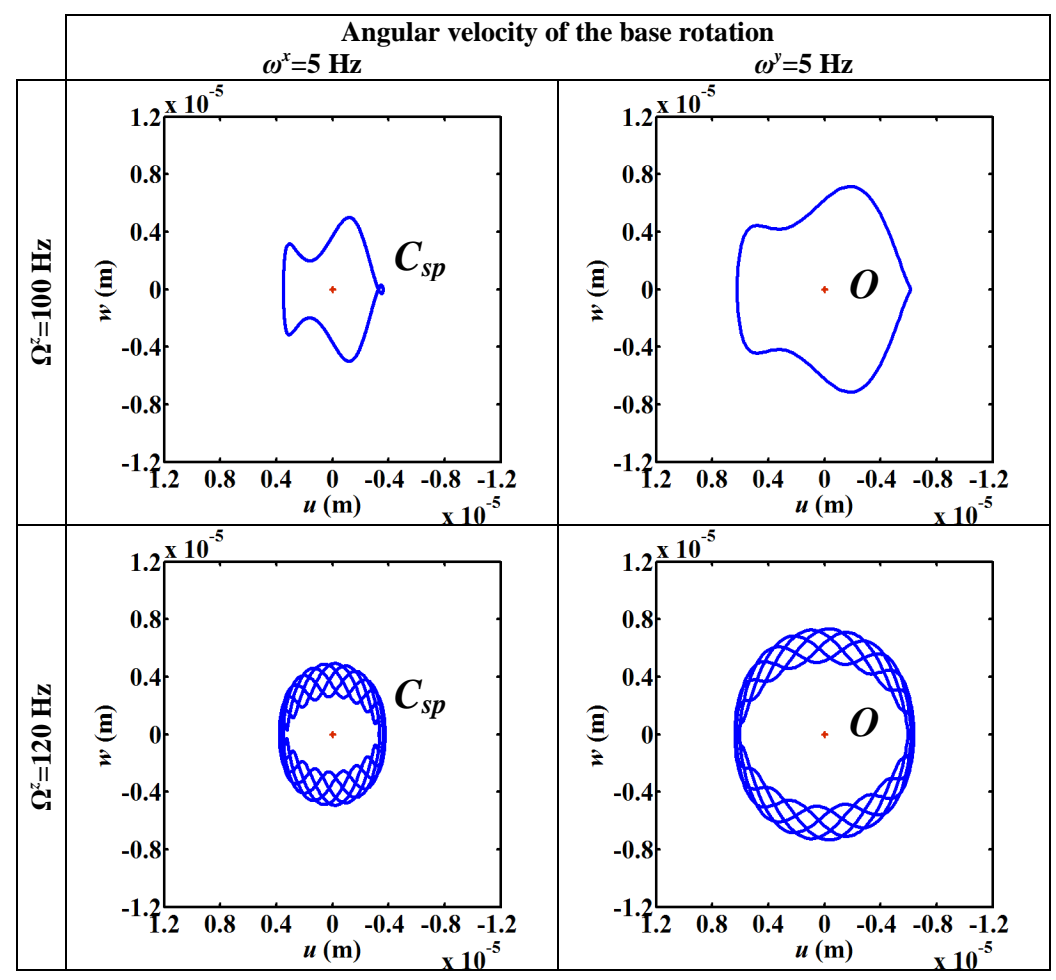


Figure 12. Influence of the frequency $\Omega^{z}$ of the base translation: $z_{O}=Z_{O} \sin \left(\Omega^{z} t\right)$ with $Z_{O}=1 \times 10^{-6} \mathrm{~m}$. Orbits at the middle of the rotor running at $\Omega=1500 \mathrm{rpm}$ and subjected to two angular velocities of the base:

$\omega^{x}=5 \mathrm{~Hz}$ or $\omega^{y}=5 \mathrm{~Hz}$.

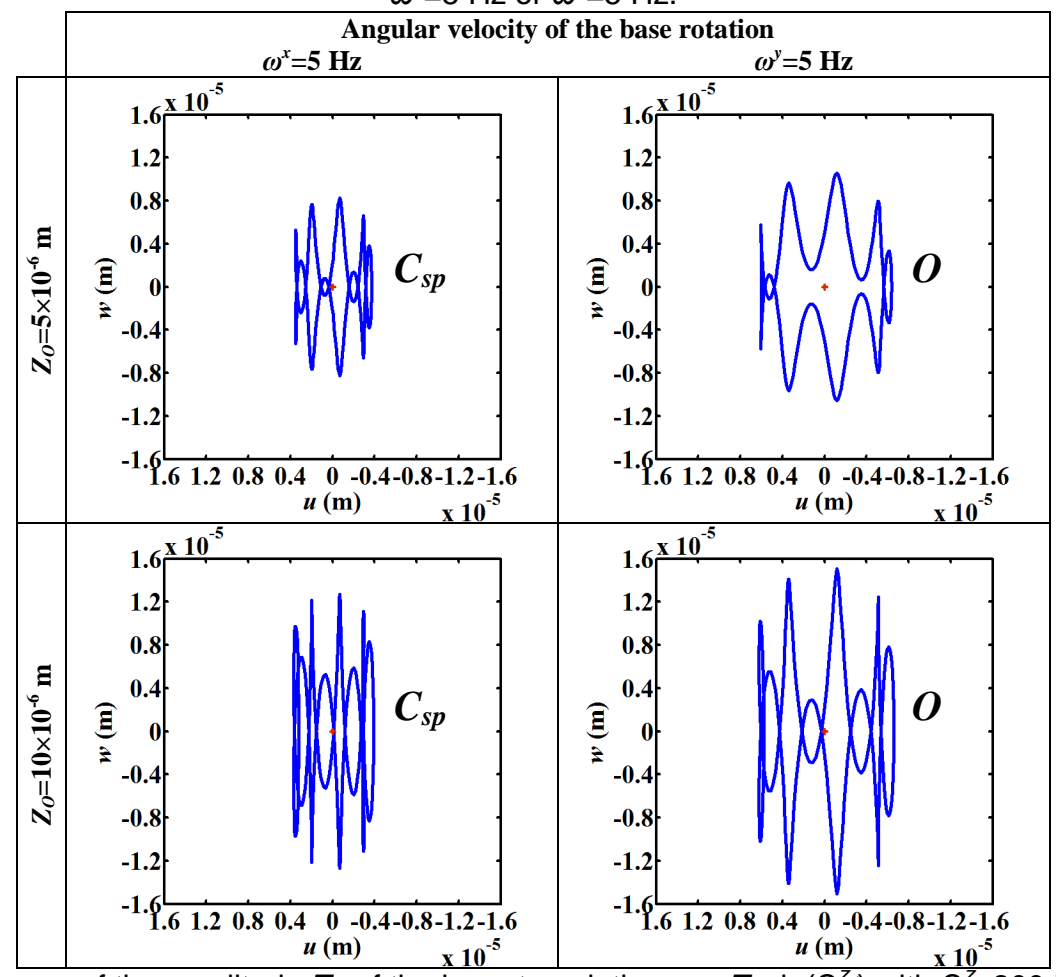

Figure 13. Influence of the amplitude $Z_{O}$ of the base translation: $z_{O}=Z_{O} \sin \left(\Omega^{2} t\right)$ with $\Omega^{2}=200 \mathrm{~Hz}$. Orbits at the middle of the rotor running at $\Omega=1500 \mathrm{rpm}$ and subjected to two angular velocities of the base: $\omega^{x}=5 \mathrm{~Hz}$ or $\omega^{y}=5 \mathrm{~Hz}$.

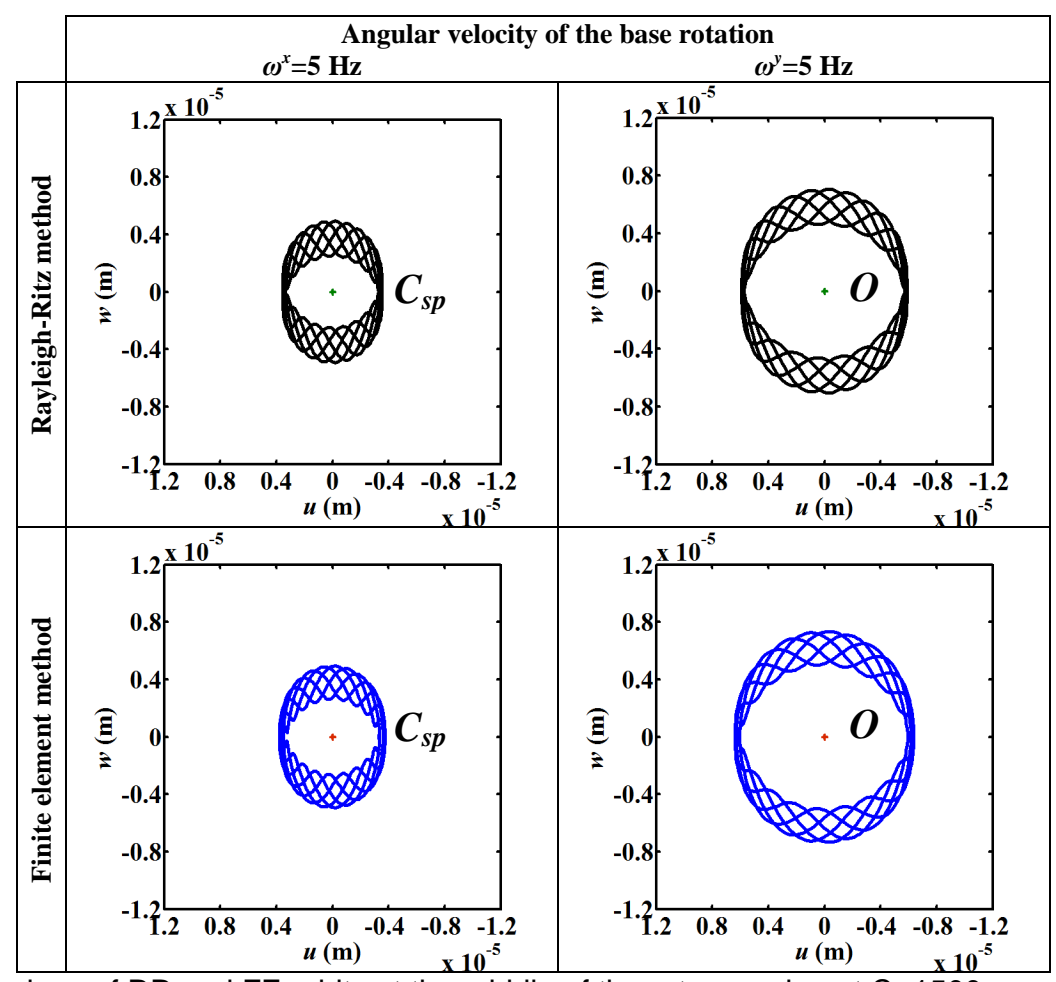

Figure 14. Comparison of RR and FE orbits at the middle of the rotor running at $\Omega=1500 \mathrm{rpm}$ and subjected to a base translation: $z_{O}=Z_{O} \sin \left(\Omega^{2} t\right)$ with $Z_{O}=1 \times 10^{-6} \mathrm{~m}$ and $\Omega^{z}=120 \mathrm{~Hz}$, combined with two angular velocities of the base: $\omega^{x}=5 \mathrm{~Hz}$ or $\omega^{y}=5 \mathrm{~Hz}$. 


\subsection{Asymmetric rotor}

\subsubsection{Configuration and data}

The description of the asymmetric rotating rotor is similar to that in Section 7.1 but the disk is asymmetric and its geometric properties change. The shape of the disk presented in Figure 15 is defined as a function of the angle $\theta_{d}$ with the $C^{0} x$ axis of the frame $R$. The rotor data are the same as given in Table 1 . The rotor is subjected to the same excitations as indicated in the previous section. The shaft is discretized into 12 identical two-node Timoshenko beam FEs. The disk as well as the rigid short bearings \# 1 and \# 2 are located at nodes 5, 1 and 13 respectively. Thus the degrees of freedom of the bearing nodes are eliminated. The linear differential equations of motion have time-varying periodic parametric terms. In order to treat them and predict the steady-state behavior, the Runge-Kutta and Newmark time-step integration methods are used. The proportional Rayleigh damping $\mathbf{C}_{d, s h}^{i d}=\alpha^{R d} \mathbf{M}_{d, s h}+\beta^{R d} \mathbf{K}_{s h}^{e}$ of the rotating parts (disk and shaft) is taken into account (see Spears and Jensen, 2009) and its corresponding coefficients estimated for a modal damping factor $\xi^{m d}=0.03$ are given by $\alpha^{R d}=12.43$ and $\beta^{R d}=5.66 \times 10^{-5}$. The dynamic analysis is performed by means of stability charts, rotor orbits and fast Fourier transforms (FFTs). In addition, the equations of motion of the on-board rotor (see equations (36) and (38)) show that the base rotations have a parametric influence on these equations, while the base translations have only an influence on the external force vector. Therefore the overall behavior is evaluated as a function of the base rotation.

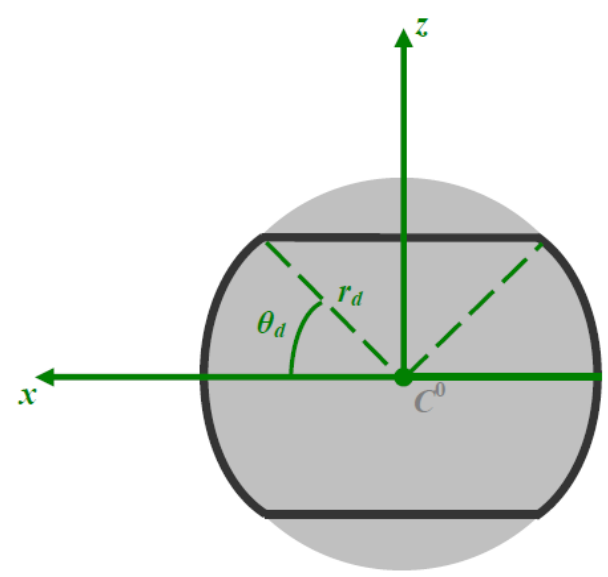

Figure 15. Geometry of the asymmetric disk.

\subsubsection{Stability charts}

The instability coming from the parametric excitation due to the geometric asymmetry of the rotor can be related to the base rotations, see equations (36) and (38). For a certain angular velocity of the rotor base, two factors affect the parametric excitations generated in the asymmetric rotor: the speed of rotation $\Omega$ of the rotor and the moment of inertia $I_{m_{d}}^{d i}$. The latter depends on the angle $\theta_{d}$ describing the geometric asymmetry of the disk. Therefore the stability analysis covers the two parameters $\Omega$ as well as $\theta_{d}$ and the stability charts of the rotor shown in Figures 16 and 17 are established for the primary resonance with $\Delta \theta_{d}=5^{\circ}$ and $\Delta \Omega=5 \mathrm{rpm}$. The zones of instability are bounded by transition curves. The increase of the base angular velocity $\omega^{x}$ roughly does not change the size of the instability zones and slightly shifts them down (see Figure 16). For the considered values of the angle $\theta_{d}$, the on-board rotor can become unstable for $\Omega \in[2980 ; 3715 \mathrm{rpm}]$ and $\omega^{x}=5 \mathrm{~Hz}$ as well as for $\Omega \in[2965 ; 3700 \mathrm{rpm}]$ and $\omega^{x}=10 \mathrm{~Hz}$. In the same way, the increase of $\omega^{y}$ does not change the size of the instability zones but this time, their limits are remarkably shifted down (see Figure 17). For the considered values of $\theta_{d}$, the rotor can be unstable for $\Omega \in[2690 ; 3420 \mathrm{rpm}]$ and $\omega^{y}=5 \mathrm{~Hz}$ as well as for $\Omega \in[2390 ; 3120 \mathrm{rpm}]$ and $\omega^{y}=10 \mathrm{~Hz}$.

The stability chart plotted in Figure 18(a) is predicted with the RR method. Figure 18(b) compares the bounds of the primary instability regions calculated by the RR and FE methods. As can be seen in this figure, the bounds predicted by the RR method are $4-7 \%$ greater than those predicted by the FE method and the FE instability region is narrower. 


\subsubsection{Orbits}

\subsubsection{Pure rotation $\omega^{x}$ (or $\left.\omega^{y}\right)$ of the base}

Figure 19(a) presents the mass unbalance orbit at the middle of the asymmetric rotor (node 7) for an angle of disk asymmetry $\theta_{d}=45^{\circ}, \Omega=1500 \mathrm{rpm}(=25 \mathrm{~Hz})$ and a fixed base (i.e., $\left.\omega^{x}=\omega^{y}=0\right)$. The predicted orbit is circular but slightly smaller than that of the symmetric rotor because the mass and the inertia of the rotor are reduced. The predicted orbit corresponding to the base angular velocity $\omega^{y}=5 \mathrm{~Hz}$ is also slightly smaller, see Figure 19(c). On the opposite side, the predicted orbit corresponding to $\omega^{x}=5 \mathrm{~Hz}$ is much greater than that of the symmetric rotor due to high external forces as explained in the next section, see Figure 19(b).

(a)

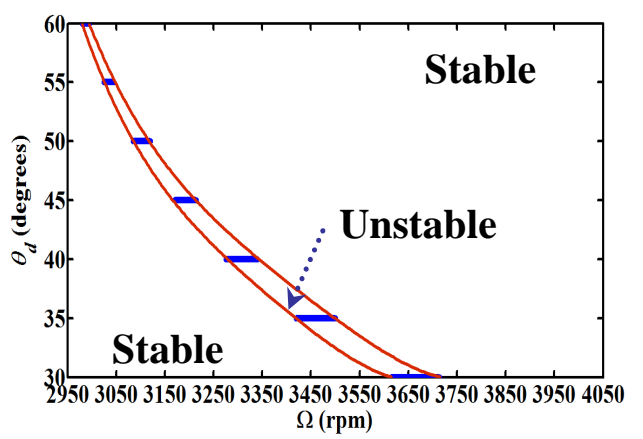

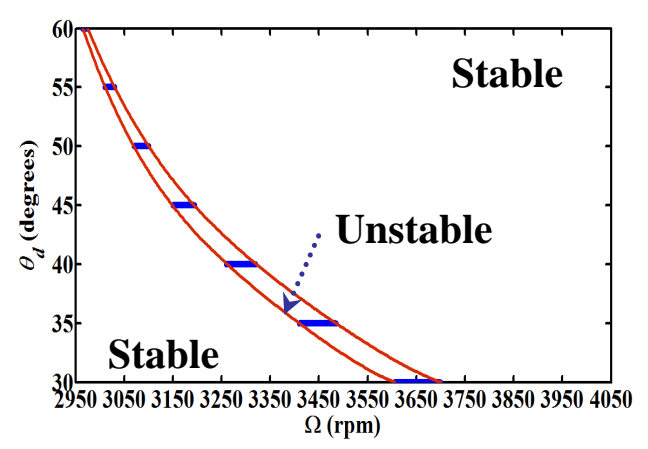

(b)$$
\Omega(\mathrm{rpm})
$$

Figure 16. Stability charts of the rotor due to two base angular velocities: (a) $\omega^{x}=5 \mathrm{~Hz}$, (b) $\omega^{x}=10 \mathrm{~Hz}$.

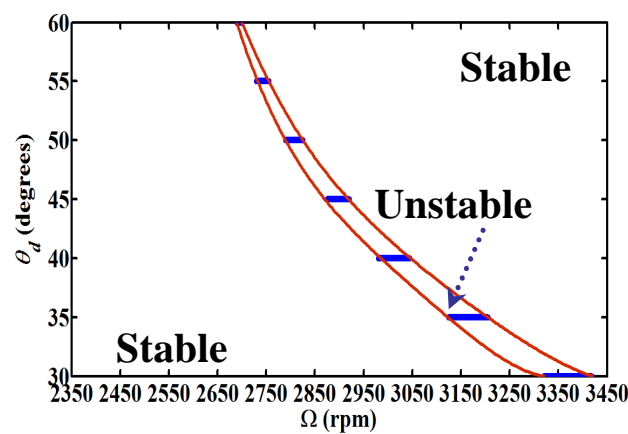

(b)

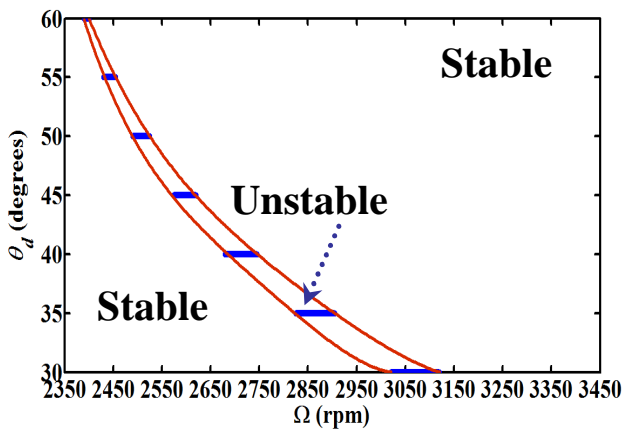

Figure 17. Stability charts of the rotor due to two base angular velocities: (a) $\omega^{y}=5 \mathrm{~Hz}$, (b) $\omega^{y}=10 \mathrm{~Hz}$.

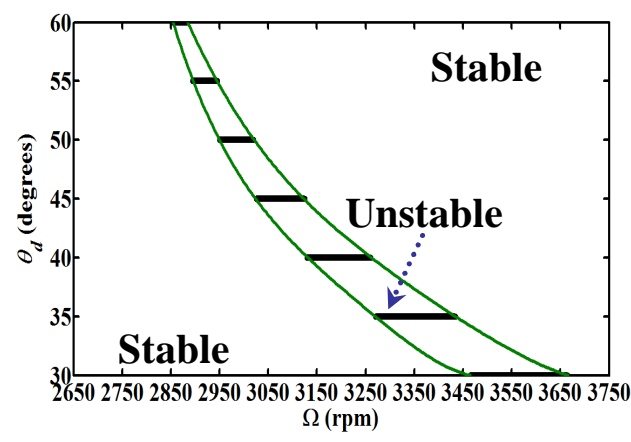

(b)

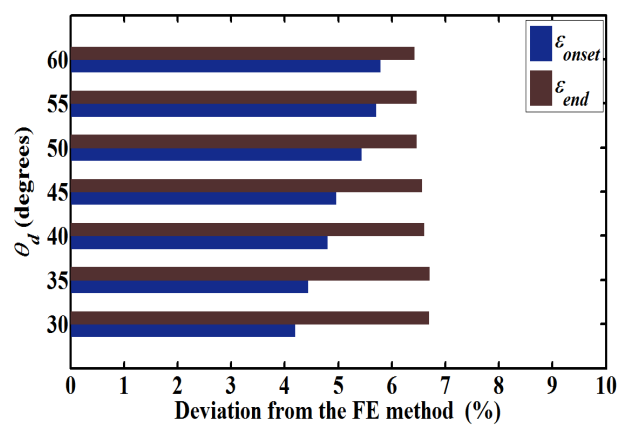

a)

chart of the rotor due to a base angular velocity $\omega^{y}=5 \mathrm{~Hz}$
(b) Deviations of onsets and ends of the instability zones.
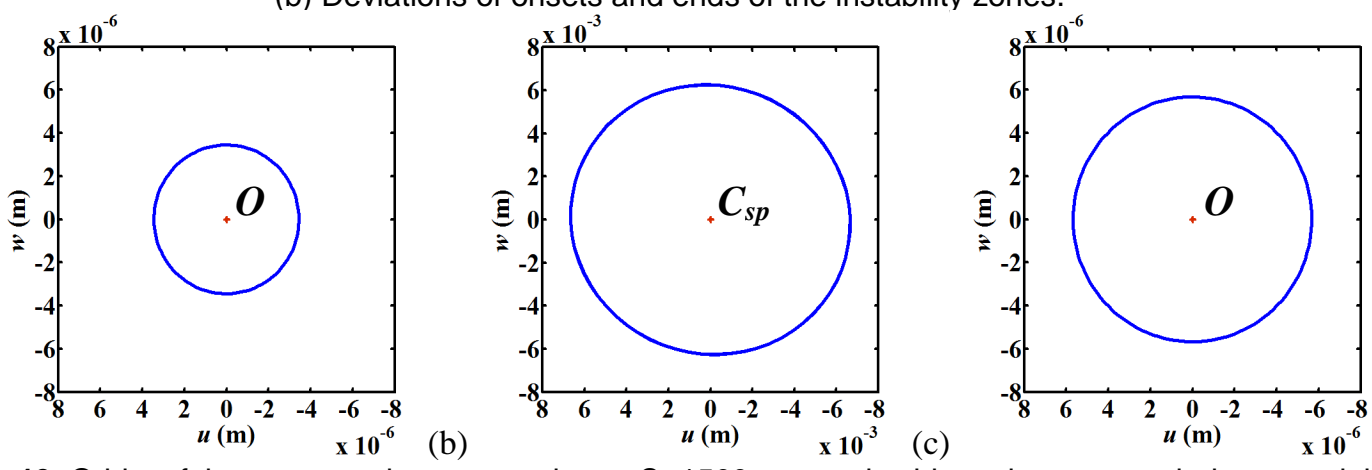

Figure

(b)

values of base rotation: (a) $\omega^{x}=\omega^{y}=0$, i.e., fixed base, (b) $\omega^{x}=5 \mathrm{~Hz}, \omega^{y}=0$, (c) $\omega^{x}=0, \omega^{y}=5 \mathrm{~Hz}$. 


\subsubsection{Combination of translation $z_{O}=Z_{O} \sin \Omega^{z} t$ and rotation $\omega^{x}\left(\right.$ or $\left.\omega^{y}\right)$ of the base}

Since the equations of motion are linear with periodic parametric coefficients (see equations (36) and (38)), the steady-state response to the mass unbalance is superimposed with the steady-state response to the base excitations. Therefore the orbit characteristics are related to both excitations. Figure 20 presents the orbits and their FFTs at the middle of the asymmetric rotor (node 7) for $\theta_{d}=45^{\circ}, \Omega=1500 \mathrm{rpm}(=25 \mathrm{~Hz}$ ) and combined rotational and translational excitations of the rotor base. In this figure, the base rotation and the amplitude of the harmonic base translation are kept constant ( $\omega^{x}$ or $\omega^{y}=5 \mathrm{~Hz}$ and $Z_{O}=1 \times 10^{-6} \mathrm{~m}$ ), while the frequency of the base translation varies $\left(\Omega^{z}=100 \mathrm{~Hz}, 120 \mathrm{~Hz}\right.$ and $200 \mathrm{~Hz}$ ). When the rotor is excited by the base angular velocity $\omega^{x}=5 \mathrm{~Hz}$, the stabilized orbits at the middle of the shaft are elliptical and the displacement amplitudes are mostly the same as shown in Section 7.2.3.1 whatever the value of the base translation frequency $\Omega^{z}$. FFTs exhibit only one frequency component equal to $50 \mathrm{~Hz}=2 \Omega$ because of the presence of $\omega^{x}$ in the $\cos 2 \Omega t$ and $\sin 2 \Omega t$ terms of the external excitation produced by the geometric asymmetry of the rotor, which are prevalent regarding the other forcing terms, see equation (36). When the rotor is excited by the base angular velocity $\omega^{y}=5 \mathrm{~Hz}$, the frequency of the harmonic base translation has a limited influence on the magnitudes of the orbits. In addition, the stabilized orbits have shapes more complex than those obtained for the symmetric rotor (see Figure 12) since they involve frequency components due to the mass unbalance excitation (25 Hz), to the harmonic base translations $(100 \mathrm{~Hz}, 120 \mathrm{~Hz}$ and $200 \mathrm{~Hz})$ and to the combination of frequencies of both excitations as shown in FFTs of Figure 20.

The RR and FE orbits seen in Figure 21 have the same shape but quantitatively are different. The $x$ and $z$ magnitude deviations are $\varepsilon_{u}=-36.74 \%$ and $\varepsilon_{w}=-36.96 \%$ for $\omega^{x}$ as well as $\varepsilon_{u}=-5.26 \%$ and $\varepsilon_{w}=3.36 \%$ for $\omega^{y}$. The static position coordinate deviation is $\varepsilon_{s p}=-6.98 \%$. The observed deviations are due to the fact that the RR expansion is based only on two generalized coordinates, while the FE model is built with 48 degrees of freedom, i.e., the FE model is more flexible because it uses more degrees of freedom than the RR model.

\section{Conclusions}

The dynamics of two different rotor configurations (symmetric and asymmetric), whose rigid base is subjected to combined constant rotation and sinusoidal translation, are analytically and numerically investigated. A new finite element model based on the Timoshenko beam theory is presented to analyze the steady-state dynamic behavior of the on-board rotor mounted on rigid bearings. It contains the effects associated with the rotary inertia, the gyroscopic inertia, the shear deformation of shaft, the geometric asymmetry of disk and/or shaft as well as six types of deterministic motions (rotations and translations) of the rotor rigid base. Moreover, the rotational effects and the geometric stiffening effects relative to the centrifugal stressing caused by the base rotations are taken into account. The derivation of the equations of motion has shown that the base rotation brings not only a parametric contribution to them but also additional external force vectors, while the base translation only adds external force vectors.

In the case of the symmetric rotor, the equations of motion always are ordinary differential equations with constant coefficients. A dynamic instability can occur in the rotor due to the presence of negative diagonal terms containing the angular velocities of the base in the symmetric stiffness matrices which can lose their positive definiteness. It is shown that the base rotations change not only the natural frequencies of the rotor but also can break the symmetry of the dynamic behavior in the case of the rotation around a transverse axis. The sinusoidal base translation does not change the natural frequencies of the rotor. It has only an influence on the corresponding response amplitudes as well as on the magnitude and the shape of the rotor orbits.

The asymmetric rotor introduces time-varying periodic parametric coefficients in the linear equations of motion. Thus possible regions of instability of the rotor can occur and their sizes cannot be related to the base rotations. On the other hand, the base rotations shift the instability regions along the speed of rotation of the rotor. The sinusoidal base translation has no influence on the instability regions but only an influence on the magnitude and the shape of the rotor orbits.

The finite element calculations have been validated against the Rayleigh-Ritz formulation. Using the finite element method is useful because it is more accurate and can be used for industrial rotating machinery modeling. 


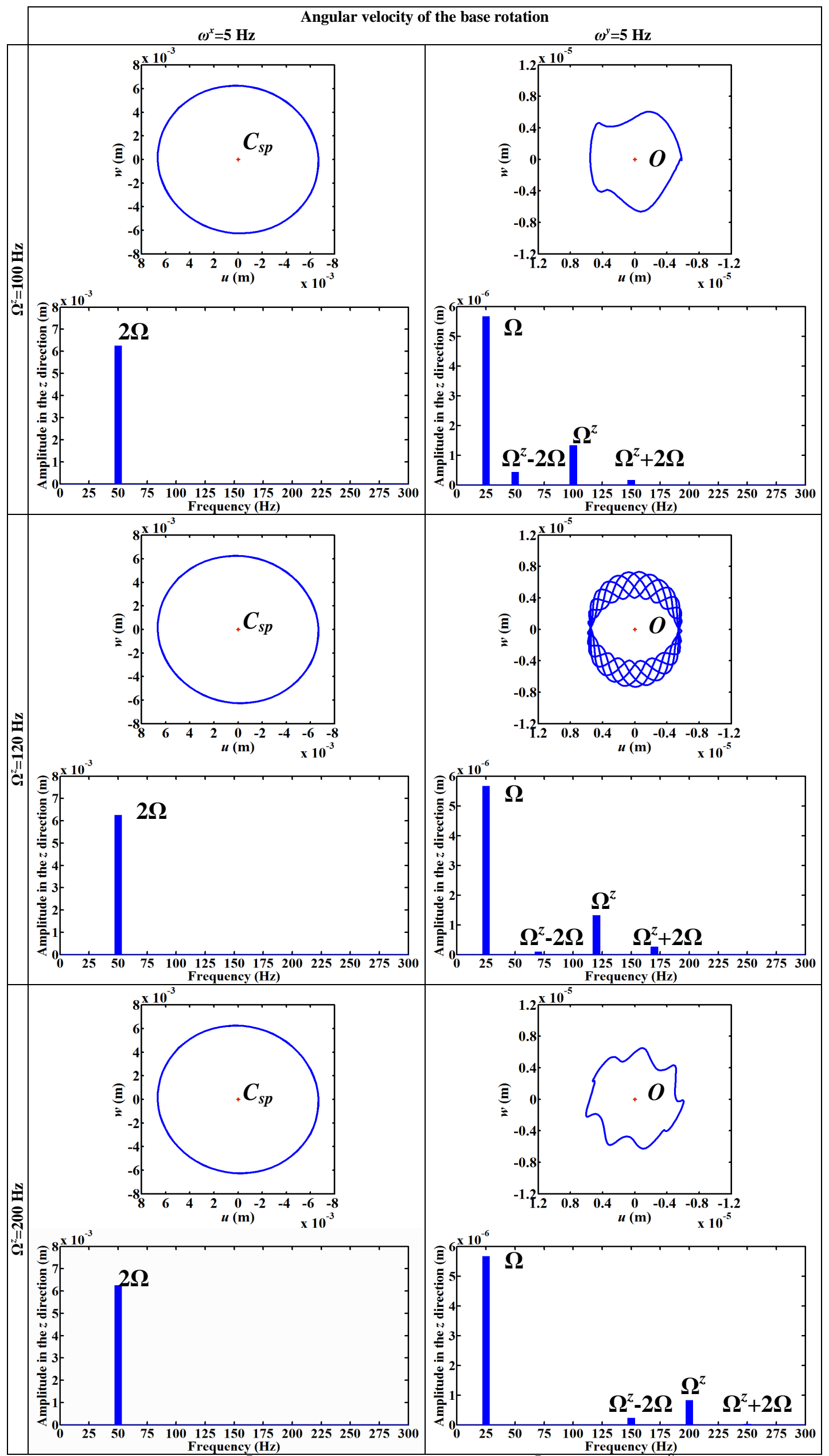

Figure 20. Influence of the frequency $\Omega^{2}$ of the base translation: $z_{O}=Z_{O} \sin \left(\Omega^{2} t\right)$ with $Z_{O}=1 \times 10^{-6} \mathrm{~m}$. Orbits of the asymmetric rotor running at $\Omega=1500 \mathrm{rpm}$ and subjected to two angular velocities of the base: $\omega^{x}=5 \mathrm{~Hz}$ or $\omega^{y}=5 \mathrm{~Hz}$. 


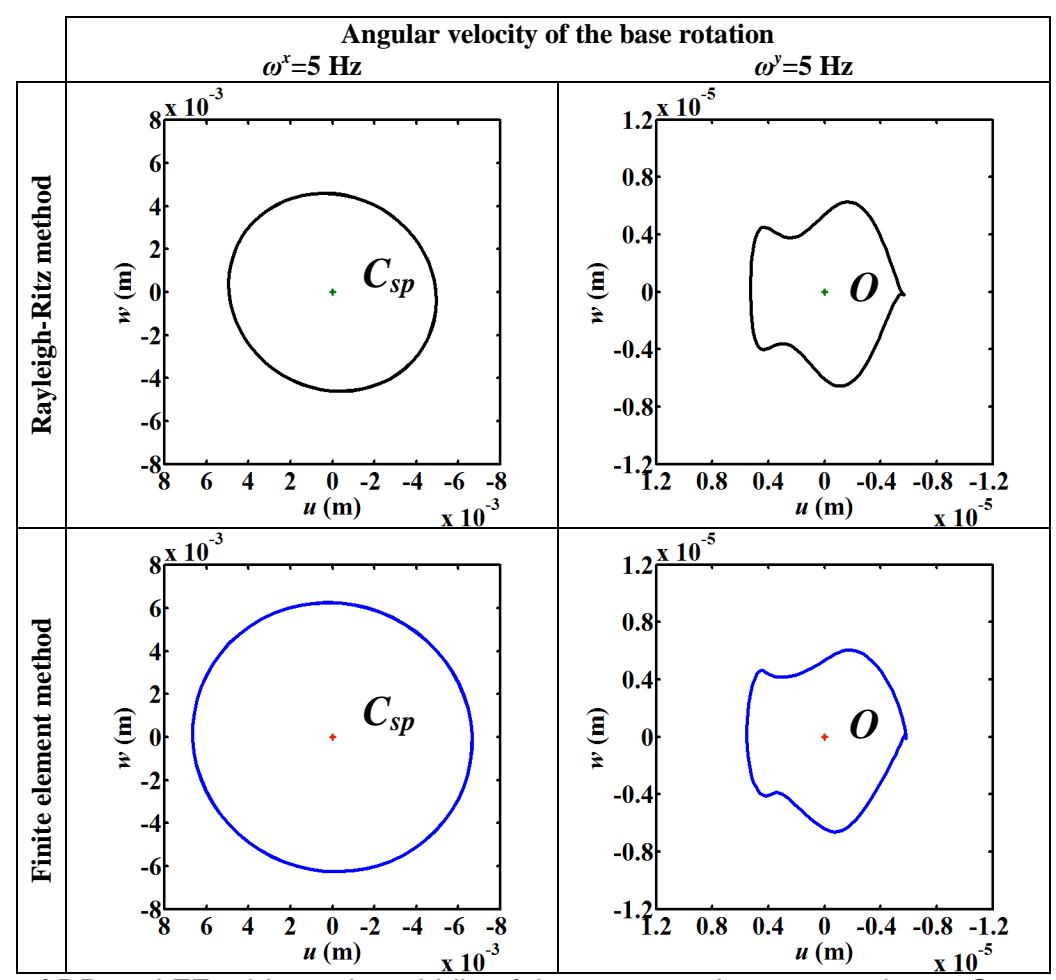

Figure 21. Comparison of RR and FE orbits at the middle of the asymmetric rotor running at $\Omega=1500$ rpm and subjected to a base translation: $z_{O}=Z_{O} \sin \left(\Omega^{z} t\right)$ with $Z_{O}=1 \times 10^{-6} \mathrm{~m}$ and $\Omega^{z}=100 \mathrm{~Hz}$, combined with two angular velocities of the base: $\omega^{x}=5 \mathrm{~Hz}$ or $\omega^{y}=5 \mathrm{~Hz}$.

\section{References}

Bachelet L, Driot N and Ferraris G (2006) Rotors under seismic excitation: A spectral approach. In: Proceedings of IFToMM 7th International Conference on Rotor Dynamics, Vienna, Austria, 25-28 September.

Beley-Sayettat A (1994) Effet des dissymétries et effet sismique en dynamique des rotors (in French). PhD Thesis, INSALyon, France.

Berlioz A, Dufour R and Sinha SC (2000) Bifurcation in a nonlinear autoparametric system using experimental and numerical investigations. Nonlinear Dynamics 23(2): 175-187.

Cavalca KL, Cavalcante PF and Okabe EP (2005) An investigation on the influence of the supporting structure on the dynamics of the rotor system. Mechanical Systems and Signal Processing 19(1): 157-174.

Chen L-W and Ku D-M (1992) Dynamic stability of a cantilever shaft-disk system. ASME Journal of Vibration and Acoustics 114(3): 326-329.

Das AS, Dutt JK and Ray K (2010) Active vibration control of unbalanced flexible rotor-shaft systems parametrically excited due to base motion. Applied Mathematical Modelling 34(9): 2353-2369.

Driot N, Lamarque CH and Berlioz A (2006) Theoretical and experimental analysis of a base-excited rotor. ASME Journal of Computational and Nonlinear Dynamics 1(3): 257-263.

Duchemin M (2003) Contribution à l'étude du comportement dynamique d'un rotor embarqué (in French). PhD Thesis, INSA-Lyon, France.

Duchemin M, Berlioz A and Ferraris G (2006) Dynamic behavior and stability of a rotor under base excitation. ASME Journal of Vibration and Acoustics 128(5): 576-585.

Dufour R and Berlioz A (1998) Parametric instability of a beam due to axial excitations and to boundary conditions. ASME Journal of Vibration and Acoustics 120(2): 461-467.

Edwards S, Lees AW and Friswell MI (2000) Experimental identification of excitation and support parameters of a flexible rotor-bearings-foundation system from a single run-down. Journal of Sound and Vibration 232(5): 963992.

El-Saeidy FMA and Sticher F (2010) Dynamics of a rigid rotor linear/nonlinear bearings system subject to rotating unbalance and base excitations. Journal of Vibration and Control 16(3): 403-438.

Evan-Iwanowski RM (1976) Resonance Oscillations in Mechanical Systems. New York: Elsevier.

Feng N and Hahn E (2011) Rotor-model-based identification of foundations in rotating machinery using modal parameters. In: Proceedings of the Tenth International Conference on Vibration Problems, Prague, Czech Republic, 5-8 September.

Gaganis BJ, Zisimopoulos AK, Nikolakopoulos PG and Papadopoulos CA (1999) Modal analysis of rotor on piecewise linear journal bearings under seismic excitation. ASME Journal of Vibration and Acoustics 121(2): 190-196. 
Genta G (2005) Dynamics of Rotating Systems. New York: Springer.

Guilhen PM, Berthier P, Ferraris G and Lalanne M (1988) Instability and unbalance response of dissymmetric rotorbearing systems. ASME Journal of Vibration, Acoustics, Stress and Reliability in Design 110(3): 288-294.

Hori Y and Kato T (1990) Earthquake-induced instability of a rotor supported by oil film bearings. ASME Journal of Vibration and Acoustics 112(2): 160-165.

Kang Y, Shih Y-P and Lee A-C (1992) Investigation on the steady-state responses of asymmetric rotors. ASME Journal of Vibration and Acoustics 114(2): 194-208.

Kang Y, Chang Y-P, Tsai J-W, Mu L-H and Chang Y-F (2000) An investigation in stiffness effects on dynamics of rotorbearing-foundation systems. Journal of Sound and Vibration 231(2): 343-374.

Kang Y, Tseng M-H, Wang S-M, Chiang C-P and Wang C-C (2003) An accuracy improvement for balancing crankshafts. Mechanism and Machine Theory 38(12): 1449-1467.

Lalanne M and Ferraris G (1998) Rotordynamics Prediction in Engineering. Chichester: Wiley.

Lee AS, Kim BO and Kim Y-C (2006) A finite element transient response analysis method of a rotor-bearing system to base shock excitations using the state-space Newmark scheme and comparisons with experiments. Journal of Sound and Vibration 297(3-5): 595-615.

Levecque N, Mahfoud J, Violette D, Ferraris G and Dufour R (2011) Vibration reduction of a single cylinder reciprocating compressor based on multi-stage balancing. Mechanism and Machine Theory 46(1): 1-9.

Oncescu F, Lakis AA and Ostiguy G (2001) Investigation of the stability and steady state response of asymmetric rotors, using finite element formulation. Journal of Sound and Vibration 245(2): 303-328.

Rao JS (1992) Rotor Dynamics. New York: Wiley.

Sabuncu M and Evran K (2006) The dynamic stability of a rotating asymmetric cross-section Timoshenko beam subjected to lateral parametric excitation. Finite Elements in Analysis and Design 42(5): 454-469.

Spears RE and Jensen SR (2009) Approach for selection of Rayleigh damping parameters used for time history analysis. In: Proceedings of the ASME 2009 Pressure Vessels and Piping Division Conference, Prague, Czech Republic, 26-30 July.

Suarez LE, Singh MP and Rohanimanesh MS (1992) Seismic response of rotating machines. Earthquake Engineering and Structural Dynamics 21(1): 21-36.

Tan TH, Lee HP and Leng GSB (1997) Dynamic stability of a radially rotating beam subjected to base excitation. Computer Methods in Applied Mechanics and Engineering 146(3-4): 265-279.

\section{Appendix A: Elementary matrices and vectors for the disk, the shaft and the mass unbalance}

$$
\begin{aligned}
& \mathbf{M}_{d, s h}=\mathbf{M}_{e d}^{t r}+\mathbf{M}_{e d}^{r o}+\sum_{i=1}^{n_{\text {esh }}} \mathbf{M}_{e s h_{i}}^{t r}+\sum_{i=1}^{n_{c h t}} \mathbf{M}_{e s h_{i}}^{r o} ; \quad \mathbf{M}_{d, s h}^{c}=\mathbf{M}_{e d}^{c}+\sum_{i=1}^{n_{c s h}} \mathbf{0} ; \quad \mathbf{M}_{d, s h}^{s}=\mathbf{M}_{e d}^{s}+\sum_{i=1}^{n_{c c h}} \mathbf{0} .
\end{aligned}
$$

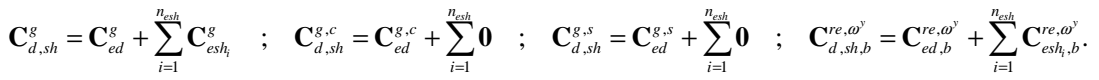

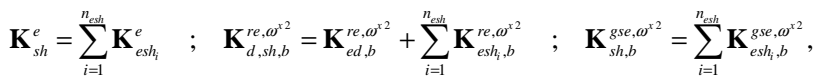

$$
\begin{aligned}
& \mathbf{K}_{d, s h, b}^{r e, \Omega \omega^{y}}=\mathbf{K}_{e d, b}^{r e, \Omega \omega^{y}}+\sum_{i=1}^{n_{\text {esh }}} \mathbf{K}_{e s h_{i}, b}^{r e, \Omega \omega^{y}} \quad ; \quad \mathbf{K}_{d, s h, b}^{r e, \omega^{\prime 2}}=\mathbf{K}_{e d, b}^{r e, \omega^{\prime 2}}+\sum_{i=1}^{n_{\text {ech }}} \mathbf{K}_{e s h_{i}, b}^{r e, \omega^{y^{2}},},
\end{aligned}
$$

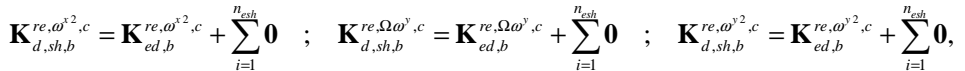

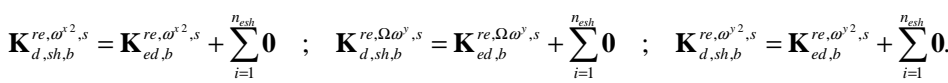

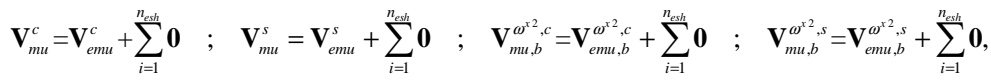

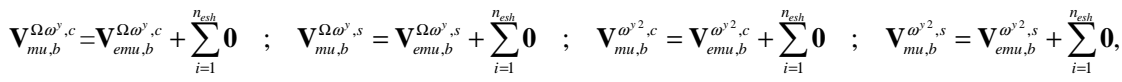

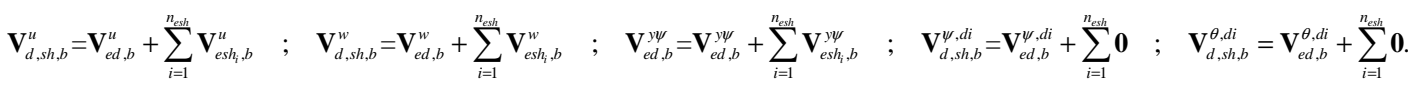


The symbol $\sum$ stands for the assembly operator after following an appropriate globalization way. The elementary matrices and vectors for the disk are defined by

$$
\begin{aligned}
& \mathbf{M}_{e d}^{t r}=\left[\begin{array}{cccc}
m_{d} & 0 & 0 & 0 \\
0 & m_{d} & 0 & 0 \\
0 & 0 & 0 & 0 \\
0 & 0 & 0 & 0
\end{array}\right] ; \quad \mathbf{M}_{e d}^{r o}=\left[\begin{array}{cccc}
0 & 0 & 0 & 0 \\
0 & 0 & 0 & 0 \\
0 & 0 & I_{m_{d}}^{m o} & 0 \\
0 & 0 & 0 & I_{m_{d}}^{m o}
\end{array}\right] ; \quad \mathbf{M}_{e d}^{c}=\left[\begin{array}{cccc}
0 & 0 & 0 & 0 \\
0 & 0 & 0 & 0 \\
0 & 0 & I_{m_{d}}^{d i} & 0 \\
0 & 0 & 0 & -I_{m_{d}}^{d i}
\end{array}\right] ; \quad \mathbf{M}_{e d}^{s}=\left[\begin{array}{cccc}
0 & 0 & 0 & 0 \\
0 & 0 & 0 & 0 \\
0 & 0 & 0 & -I_{m_{d}}^{d i} \\
0 & 0 & -I_{m_{d}}^{d i} & 0
\end{array}\right] . \\
& \mathbf{C}_{e d}^{g}=\left[\begin{array}{cccc}
0 & 0 & 0 & 0 \\
0 & 0 & 0 & 0 \\
0 & 0 & 0 & -I_{m_{d}}^{y} \\
0 & 0 & I_{m_{d}}^{y} & 0
\end{array}\right] ; \quad \mathbf{C}_{e d}^{g, c}=\left[\begin{array}{cccc}
0 & 0 & 0 & 0 \\
0 & 0 & 0 & 0 \\
0 & 0 & 0 & -2 I_{m_{d}}^{d i} \\
0 & 0 & -2 I_{m_{d}}^{d i} & 0
\end{array}\right] ; \quad \mathbf{C}_{e d}^{g, s}=\left[\begin{array}{cccc}
0 & 0 & 0 & 0 \\
0 & 0 & 0 & 0 \\
0 & 0 & -2 I_{m_{d}}^{d i} & 0 \\
0 & 0 & 0 & 2 I_{m_{d}}^{d i}
\end{array}\right] \\
& \mathbf{C}_{e d, b}^{r e, \omega^{y}}=\left[\begin{array}{cccc}
0 & 2 m_{d} & 0 & 0 \\
-2 m_{d} & 0 & 0 & 0 \\
0 & 0 & 0 & -\left(I_{m_{d}}^{y}-2 I_{m_{d}}^{m o}\right) \\
0 & 0 & I_{m_{d}}^{y}-2 I_{m_{d}}^{m o} & 0
\end{array}\right] \\
& \mathbf{K}_{e d, b}^{r e, \omega^{r 2}}=\left[\begin{array}{cccc}
0 & 0 & 0 & 0 \\
0 & -m_{d} & 0 & 0 \\
0 & 0 & 0 & 0 \\
0 & 0 & 0 & -\left(I_{m_{d}}^{y}-I_{m_{d}}^{m o}\right)
\end{array}\right] ; \quad \mathbf{K}_{e d, b}^{r e, \Omega \omega^{y}}=\left[\begin{array}{cccc}
0 & 0 & 0 & 0 \\
0 & 0 & 0 & 0 \\
0 & 0 & I_{m_{d}}^{y} & 0 \\
0 & 0 & 0 & I_{m_{d}}^{y}
\end{array}\right] ; \quad \mathbf{K}_{e d, b}^{r e, \omega^{y 2}}=\left[\begin{array}{cccc}
-m_{d} & 0 & 0 & 0 \\
0 & -m_{d} & 0 & 0 \\
0 & 0 & I_{m_{d}}^{y}-I_{m_{d}}^{m o} & 0 \\
0 & 0 & 0 & I_{m_{d}}^{y}-I_{m_{d}}^{m o}
\end{array}\right], \\
& \mathbf{K}_{e d, b}^{r e, v^{x^{2}, c}, c}=\left[\begin{array}{cccc}
0 & 0 & 0 & 0 \\
0 & 0 & 0 & 0 \\
0 & 0 & 0 & 0 \\
0 & 0 & 0 & I_{m_{d}}^{d i}
\end{array}\right] ; \quad \mathbf{K}_{e d, b}^{r e, \Omega \omega^{y}, c}=\left[\begin{array}{cccc}
0 & 0 & 0 & 0 \\
0 & 0 & 0 & 0 \\
0 & 0 & 2 I_{m_{d}}^{d i} & 0 \\
0 & 0 & 0 & -2 I_{m_{d}}^{d i}
\end{array}\right] ; \quad \mathbf{K}_{e d, b}^{r e, \omega^{r^{2}, c}, c}=\left[\begin{array}{cccc}
0 & 0 & 0 & 0 \\
0 & 0 & 0 & 0 \\
0 & 0 & I_{m_{d}}^{d i} & 0 \\
0 & 0 & 0 & -I_{m_{d}}^{d i}
\end{array}\right], \\
& \mathbf{K}_{e d, b}^{r e, \omega^{r^{2}, s}}=\left[\begin{array}{cccc}
0 & 0 & 0 & 0 \\
0 & 0 & 0 & 0 \\
0 & 0 & 0 & I_{m_{d}}^{d i} \\
0 & 0 & I_{m_{d}}^{d i} & 0
\end{array}\right] ; \quad \mathbf{K}_{e d, b}^{r e, \Omega \omega^{y}, s}=\left[\begin{array}{cccc}
0 & 0 & 0 & 0 \\
0 & 0 & 0 & 0 \\
0 & 0 & 0 & -2 I_{m_{d}}^{d i} \\
0 & 0 & -2 I_{m_{d}}^{d i} & 0
\end{array}\right] ; \quad \mathbf{K}_{e d, b}^{r e, \omega^{y^{2}, s}, s}=\left[\begin{array}{cccc}
0 & 0 & 0 & 0 \\
0 & 0 & 0 & 0 \\
0 & 0 & 0 & -I_{m_{d}}^{d i} \\
0 & 0 & -I_{m_{d}}^{d i} & 0
\end{array}\right] . \\
& \mathbf{V}_{e d, b}^{u}=\left\{\begin{array}{c}
m_{d} \\
0 \\
0 \\
0
\end{array}\right\} ; \quad \mathbf{V}_{e d, b}^{w}=\left\{\begin{array}{c}
0 \\
m_{d} \\
0 \\
0
\end{array}\right\} ; \quad \mathbf{V}_{e d, b}^{y \psi}=\left\{\begin{array}{c}
0 \\
0 \\
0 \\
I_{m_{d}}^{y}
\end{array}\right\} ; \quad \mathbf{V}_{e d, b}^{\psi, d i}=\left\{\begin{array}{c}
0 \\
0 \\
0 \\
I_{m_{d}}^{d i}
\end{array}\right\} ; \quad \mathbf{V}_{e d, b}^{\theta, d i}=\left\{\begin{array}{c}
0 \\
0 \\
I_{m_{d}}^{d i} \\
0
\end{array}\right\} .
\end{aligned}
$$

The elementary matrices and vectors for the shaft are given by

$$
\begin{aligned}
& \mathbf{M}_{e s h_{i}}^{t r}=\rho_{e s h_{i}} S_{e s h_{i}} \mathbf{M}_{e s h_{i}, 1} ; \quad \mathbf{M}_{e s h_{i}}^{r o}=\rho_{e s h_{i}} I_{e_{e s h}}^{m o} \mathbf{M}_{e s h_{h}, 2}, \\
& \mathbf{C}_{e s h_{i}}^{g}=2 \rho_{e s h_{i}} I_{S_{s h_{i}}}^{m o} \mathbf{M}_{e s h_{i}, 3} \quad ; \quad \mathbf{C}_{e s h_{i}, b}^{r e, w^{y}}=2 \rho_{e s h_{i}} S_{e s h_{i}} \mathbf{M}_{e s h_{i}, 4}, \\
& \mathbf{K}_{e s h_{i}}^{e}=E_{e s h_{i}} I_{s e l h_{i}}^{m o} \mathbf{M}_{e s h_{i}, 5} \quad ; \quad \mathbf{K}_{e s h_{i}, b}^{r e, \omega^{r 2}}=\rho_{e s h_{i}} S_{e s h_{i}} \mathbf{M}_{e s h_{i}, 6}+\rho_{e s h_{i}} I_{S_{e s h_{i}}^{m o}}^{m o} \mathbf{M}_{e s h_{i}, 7}, \\
& \mathbf{K}_{e s h_{i}, b}^{g s e, \omega^{r^{2}}}=\frac{\rho_{e s h_{i}} S_{e s h_{i}}\left(l_{e s h_{i}}^{2}-y_{s h}^{n_{i} 2}\right)}{2} \mathbf{M}_{e s h_{i}, 8}-\frac{\rho_{e s h_{i}} S_{e s h_{i}} y_{s h}^{n_{i}}}{2} \mathbf{M}_{e s h_{i}, 9}-\frac{\rho_{e s h_{i}} S_{e s h_{i}}}{2} \mathbf{M}_{e s h_{i}, 10} \text {, }
\end{aligned}
$$

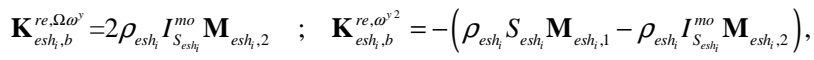

$$
\begin{aligned}
& \mathbf{V}_{e s h_{i}, b}^{u}=\rho_{e s h_{i}} S_{e s h_{i}} \mathbf{V}_{e s h_{i}, 1} \quad ; \quad \mathbf{V}_{e s h_{i}, b}^{w}=\rho_{e s h_{i}} S_{e s h_{i}} \mathbf{V}_{e s h_{i}, 2} \quad ; \quad \mathbf{V}_{e s h_{i}, b}^{y \psi}=2 \rho_{e s h_{i}} I_{e_{e s h_{i}}}^{m o} \mathbf{V}_{e s h_{i}, 3} .
\end{aligned}
$$




$$
\begin{aligned}
& \mathbf{M}_{e s h_{i}, 1}=\frac{l_{e s h_{i}}}{420\left(1+\varphi_{e s h_{i}}^{x}\right)^{2}}\left(\mathbf{M}_{e s h_{i}, 1}^{0}+\frac{7 \varphi_{e s h_{i}}^{z}}{2} \mathbf{M}_{e s h_{i}, 1}^{1}+\frac{7 \varphi_{e s h_{i}}^{z 2}}{2} \mathbf{M}_{e s h_{i}, 1}^{2}\right), \\
& \mathbf{M}_{e s h_{i}, 1}^{0}=\left[\begin{array}{cccccccc}
156 & 0 & 0 & -22 l_{e s h_{i}} & 54 & 0 & 0 & 13 l_{e s h_{i}} \\
& 156 v_{e s h_{i}}^{2} & 22 l_{e s h_{i}} v_{e s h_{i}}^{2} & 0 & 0 & 54 v_{e s h_{i}}^{2} & -13 l_{e s h_{i}} v_{e s h_{i}}^{2} & 0 \\
& & 4 l_{e s h_{i}}^{2} v_{e s h_{i}}^{2} & 0 & 0 & 13 l_{e s h_{i}} v_{e s h_{i}}^{2} & -3 l_{e s h_{i}}^{2} v_{e s h_{i}}^{2} & 0 \\
& & & 4 l_{e s h_{i}}^{2} & -13 l_{e s h_{i}} & 0 & 0 & -3 l_{e s h_{i}}^{2} \\
& & & & 156 & 0 & 0 & 22 l_{e s h_{i}} \\
S & & & & & 156 v_{e s h_{i}}^{2} & -22 l_{e s h_{i}} v_{e s h_{i}}^{2} & 0 \\
Y & & & & & & 4 l_{e s h_{i}}^{2} v_{e s h_{i}}^{2} & 0 \\
M & & & & & & & 4 l_{e s h_{i}}^{2}
\end{array}\right] \text {, }
\end{aligned}
$$

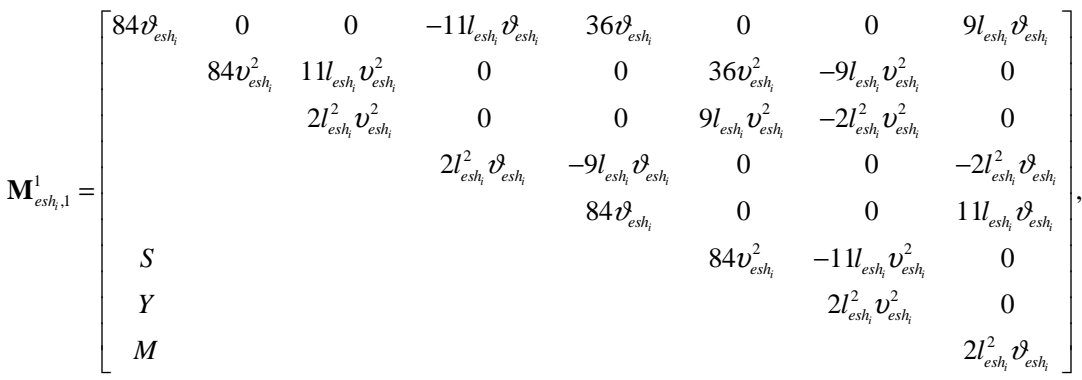

$$
\begin{aligned}
& \mathbf{M}_{e s h_{i}, 1}^{2}=\left[\begin{array}{cccccccc}
40 \vartheta_{e s h_{i}}^{2} & 0 & 0 & -5 l_{e s h_{i}} \vartheta_{e s h_{i}}^{2} & 20 \vartheta_{e s h_{i}}^{2} & 0 & 0 & 5 l_{e s h_{i}} \vartheta_{e s h_{i}}^{2} \\
& 40 v_{e s h_{i}}^{2} & 5 l_{e s h_{i}} v_{e s h_{i}}^{2} & 0 & 0 & 20 v_{e s h_{i}}^{2} & -5 l_{e s h_{i}}^{2} v_{e s h_{i}}^{2} & 0 \\
& & l_{e s h_{i}}^{2} v_{e s h_{i}}^{2} & 0 & 0 & 5 l_{e s h_{i}} v_{e s h_{i}}^{2} & -l_{e s h_{i}}^{2} v_{e s h_{i}}^{2} & 0 \\
& & & l_{e s h_{i}}^{2} \vartheta_{e s h_{i}}^{2} & -5 l_{e s h_{i}} \vartheta_{e s h_{i}}^{2} & 0 & 0 & -l_{e s h_{i}}^{2} \vartheta_{e s h_{i}}^{2} \\
& & & & 40 \vartheta_{e s h_{i}}^{2} & 0 & 0 & 5 l_{e s h_{i}}^{2} \vartheta_{e s h_{i}}^{2} \\
S & & & & & 40 v_{e s h_{i}}^{2} & -5 l_{e s h_{i}} v_{e s h_{i}}^{2} & 0 \\
Y & & & & & & l_{e s h_{i}}^{2} v_{e s h_{i}}^{2} & 0 \\
M & & & & & & & l_{e s h_{i}}^{2} \vartheta_{e s h_{i}}^{2}
\end{array}\right] \text {. } \\
& \mathbf{M}_{e s h_{i}, 2}=\frac{1}{30 l_{e s h_{i}}\left(1+\varphi_{e s h_{i}}^{x}\right)^{2}}\left(\mathbf{M}_{e s h_{i}, 2}^{0}+5 l_{e s h_{i}} \varphi_{e s h_{i}}^{z} \mathbf{M}_{e s h_{i}, 2}^{1}+5 l_{e s h_{i}}^{2} \varphi_{e s h_{i}}^{22} \mathbf{M}_{e s h_{i}, 2}^{2}\right),
\end{aligned}
$$

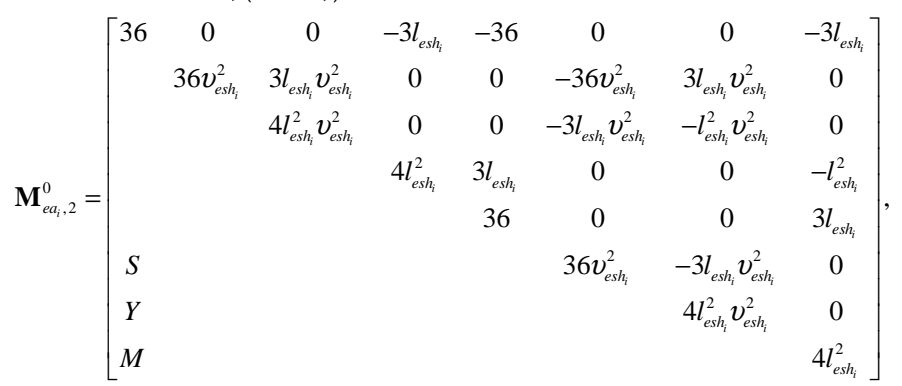

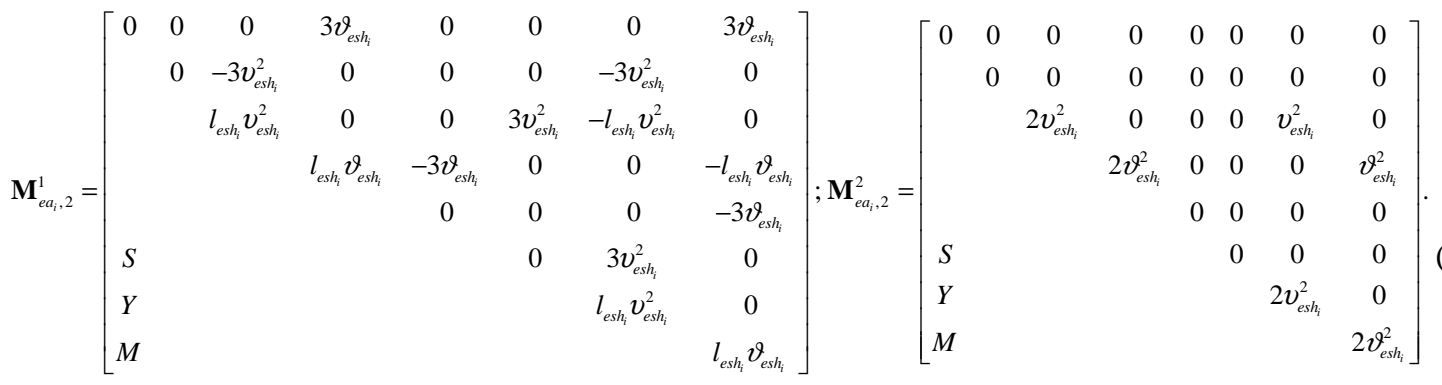




$$
\begin{aligned}
& \mathbf{M}_{e s h_{i}, 3}=\frac{1}{30 l_{e s h_{i}}\left(1+\varphi_{e s h_{i}}^{x}\right)\left(1+\varphi_{e s h_{i}}^{z}\right)}\left(\mathbf{M}_{e s h_{i}, 3}^{0}+\frac{5 l_{e s h_{i}} \varphi_{e s h_{i}}^{2}}{2} \mathbf{M}_{e s h_{i}, 3}^{1}+5 l_{e s h_{i}}^{2} \varphi_{e s h_{i}}^{x} \varphi_{e s h_{i}}^{z} \mathbf{M}_{e s h_{i}, 3}^{2}\right) \\
& \mathbf{M}_{e s h_{i}, 3}^{0}=\left[\begin{array}{cccccccc}
0 & -36 & -3 l_{e s h_{i}} & 0 & 0 & 36 & -3 l_{e s h_{i}} & 0 \\
S & 0 & 0 & -3 l_{e s h_{i}} & -36 & 0 & 0 & -3 l_{e s h_{i}} \\
K & & 0 & -4 l_{e s h_{i}}^{2} & -3 l_{e s h_{i}} & 0 & 0 & l_{e s h_{i}}^{2} \\
E & & & 0 & 0 & -3 l_{e s h_{i}} & -l_{e s h_{i}}^{2} & 0 \\
W & & & & 0 & -36 & 3 l_{e s h_{i}} & 0 \\
S & & & & & 0 & 0 & 3 l_{e s h_{i}} \\
Y & & & & & 0 & -4 l_{e s h_{i}}^{2} \\
M & & & & & & 0
\end{array}\right], \\
& \mathbf{M}_{e s h_{i}, 3}^{1}=\left[\begin{array}{cccccccc}
0 & 0 & 6 & 0 & 0 & 0 & 6 & 0 \\
S & 0 & 0 & 6 \vartheta_{e s h_{i}} & 0 & 0 & 0 & 6 \vartheta_{e s h_{i}} \\
K & 0 & -l_{e s h_{i}}\left(1+\vartheta_{e s h_{i}}\right) & 6 & 0 & 0 & l_{e s h_{i}}\left(1+\vartheta_{e s h_{i}}\right) \\
E & & 0 & 0 & 6 \vartheta_{e s h_{i}} & -l_{e s h_{i}}\left(1+\vartheta_{e s h_{i}}\right) & 0 \\
W & & & 0 & 0 & -6 & 0 \\
S & & & & 0 & 0 & -6 \vartheta_{e s h_{i}} \\
Y & & & & & 0 & -l_{e s h_{i}}\left(1+\vartheta_{e s h_{i}}\right) \\
M & & & & & & 0
\end{array}\right], \\
& \mathbf{M}_{e s h, 3}^{2}=\left[\begin{array}{cccccccc}
0 & 0 & 0 & 0 & 0 & 0 & 0 & 0 \\
S & 0 & 0 & 0 & 0 & 0 & 0 & 0 \\
K & & 0 & -2 & 0 & 0 & 0 & -1 \\
E & & & 0 & 0 & 0 & 1 & 0 \\
W & & & & 0 & 0 & 0 & 0 \\
S & 0 & & & 0 & 0 & 0 \\
Y & 0 & & & & 0 & -2 \\
M & 0 & & & & & 0
\end{array}\right] . \\
& \mathbf{M}_{e s h_{i}, 4}=\frac{l_{e s h_{i}}}{420\left(1+\varphi_{e s h_{i}}^{x}\right)\left(1+\varphi_{e s h_{i}}^{2}\right)}\left(\mathbf{M}_{e s h_{i}, 4}^{0}+\frac{7 \varphi_{e s h_{i}}^{z}}{2} \mathbf{M}_{e s h_{i}, 4}^{1}+\frac{7 \varphi_{e s h_{i}}^{x} \varphi_{e s h_{i}}^{z}}{2} \mathbf{M}_{e s h_{i}, 4}^{2}\right),
\end{aligned}
$$

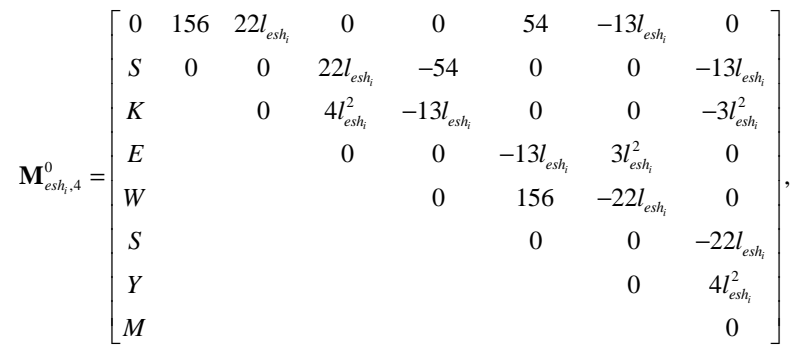

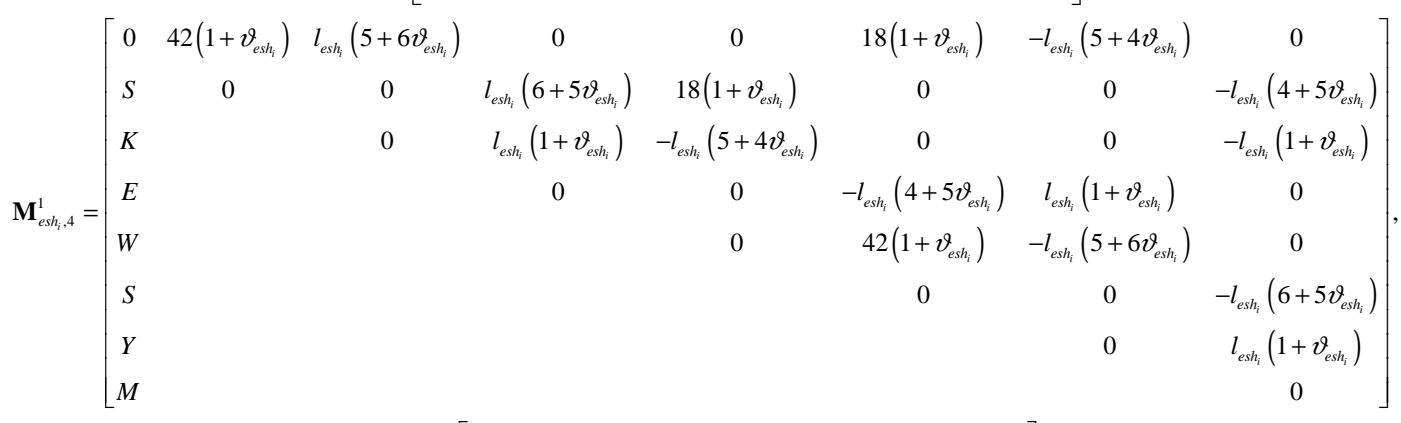

$$
\begin{aligned}
& \mathbf{M}_{e s h_{i}, 4}^{2}=\left[\begin{array}{cccccccc}
0 & 40 & 5 l_{e s h_{i}} & 0 & 0 & 10 & -5 l_{e s h_{i}} & 0 \\
S & 0 & 0 & 5 l_{e s h_{i}} & -10 & 0 & 0 & -5 l_{e s h_{i}} \\
K & & 0 & l_{e s h_{i}}^{2} & -5 l_{e s h_{i}} & 0 & 0 & -l_{e a}^{2} \\
E & & & 0 & 0 & -5 l_{e s h_{i}} & l_{e s h_{i}}^{2} & 0 \\
W & & & & 0 & 40 & -5 l_{e s h_{i}} & 0 \\
S & & & & & 0 & 0 & -5 l_{e s h_{i}} \\
Y & & & & & 0 & l_{e s h_{i}}^{2} \\
M & & & & & & 0
\end{array}\right] .
\end{aligned}
$$




$$
\begin{aligned}
& \mathbf{M}_{e s h_{i}, 5}=\frac{1}{l_{e s h_{i}}^{3}\left(1+\varphi_{e s h_{i}}^{x}\right)^{2}}\left(\mathbf{M}_{e s h_{i}, 5}^{0}+\varphi_{e s h_{i}}^{z} \mathbf{M}_{e s h_{i}, 5}^{1}+\frac{\varphi_{e s h_{i}}^{z 2}}{\varphi_{e s h_{i}}^{m o}} \mathbf{M}_{e s h_{i}, 5}^{2}\right), \\
& \mathbf{M}_{e s h_{i}, 5}^{0}=\left[\begin{array}{cccccccc}
12 & 0 & 0 & -6 l_{e s h_{i}} & -12 & 0 & 0 & -6 l_{e s h_{i}} \\
& 12 v_{e s h_{i}}^{2} & 6 l_{e s h_{i}} v_{e s h_{i}}^{2} & 0 & 0 & -12 v_{e s h_{i}}^{2} & 6 l_{e s h_{i}} v_{e s h_{i}}^{2} & 0 \\
& & 4 l_{e s h_{i}}^{2} v_{e s h_{i}}^{2} & 0 & 0 & -6 l_{e s h_{i}} v_{e s h_{i}}^{2} & 2 l_{e s h_{i}}^{2} v_{e s h_{i}}^{2} & 0 \\
& & & 4 l_{e s h_{i}}^{2} & 6 l_{e s h_{i}} & 0 & 0 & 2 l_{e s h_{i}}^{2} \\
& & & & 12 & 0 & 0 & 6 l_{e s h_{i}} \\
S & & & & & 12 v_{e s h_{i}}^{2} & -6 l_{e s h_{i}} v_{e s h_{i}}^{2} & 0 \\
Y & & & & & & 4 l_{e s h_{i}}^{2} v_{e s h_{i}}^{2} & 0 \\
M & & & & & & & 4 l_{e s h_{i}}^{2}
\end{array}\right] \text {, } \\
& \mathbf{M}_{e s h_{i}, 5}^{1}=\left[\begin{array}{cccccccc}
0 & 0 & 0 & 0 & 0 & 0 & 0 & 0 \\
& 0 & 0 & 0 & 0 & 0 & 0 & 0 \\
& & 2 l_{e s h_{i}}^{2} v_{e s h_{i}}^{2} & 0 & 0 & 0 & -2 l_{e s h_{i}}^{2} v_{e s h_{i}}^{2} & 0 \\
& & & 2 l_{e s h_{i}}^{2} \vartheta_{e s h_{i}} & 0 & 0 & 0 & -2 l_{e s h_{i}}^{2} \vartheta_{e s h_{i}} \\
& & & & 0 & 0 & 0 & 0 \\
S & & & & 0 & 0 & 0 \\
Y & & & & & 2 l_{e s h_{i}}^{2} v_{e s h_{i}}^{2} & 0 \\
M & & & & & & 2 l_{e s h_{i}}^{2} \vartheta_{e s h_{i}}
\end{array}\right]
\end{aligned}
$$

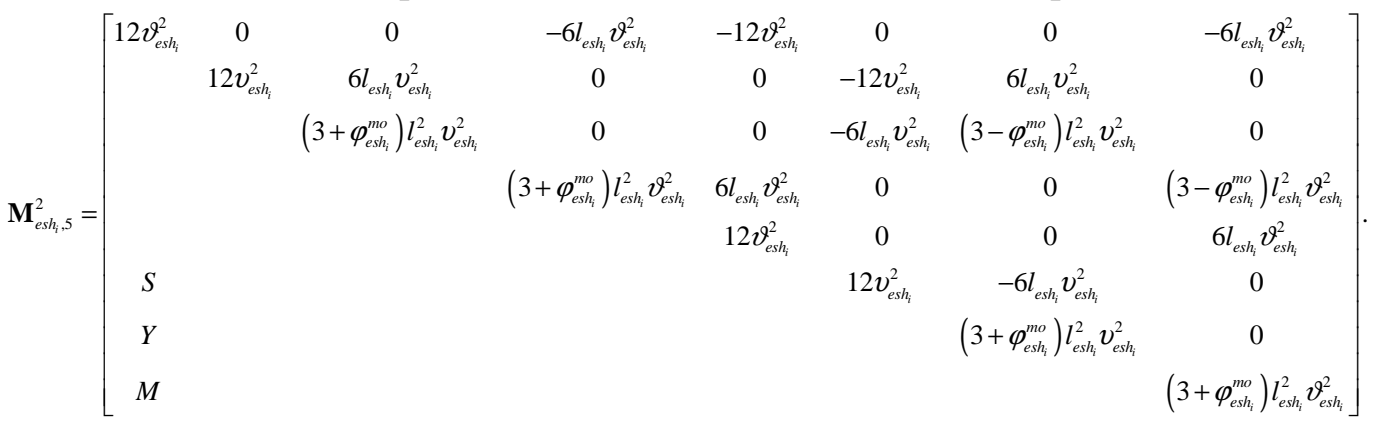

$$
\begin{aligned}
& \mathbf{M}_{e s h_{i}, 6}=\frac{l_{e s h_{i}}}{420\left(1+\varphi_{e s h_{i}}^{z}\right)^{2}}\left(\mathbf{M}_{e s h_{i}, 6}^{0}+\frac{7 \varphi_{e s h_{i}}^{z}}{2} \mathbf{M}_{e s h_{i}, 6}^{1}+\frac{7 \varphi_{e s h_{i}}^{z 2}}{2} \mathbf{M}_{e s h_{i}, 6}^{2}\right), \\
& \mathbf{M}_{e s h_{i}, 6}^{0}=\left[\begin{array}{cccccccc}
0 & 0 & 0 & 0 & 0 & 0 & 0 & 0 \\
& -156 & -22 l_{\text {esh }} & 0 & 0 & -54 & 13 l_{\text {ssh }_{i}} & 0 \\
& & -4 l_{\text {esh }}^{2} & 0 & 0 & -13 l_{\text {esh }_{i}} & 3 l_{\text {esh }}^{2} & 0 \\
& & & 0 & 0 & 0 & 0 & 0 \\
& & & & 0 & 0 & 0 & 0 \\
S & & & & & -156 & 22 l_{\text {esh }_{i}} & 0 \\
Y & & & & & -4 l_{\text {esh }}^{2} & 0 \\
M & & & & & & 0
\end{array}\right], \\
& \mathbf{M}_{e s h_{i}, 6}^{1}=\left[\begin{array}{cccccccc}
0 & 0 & 0 & 0 & 0 & 0 & 0 & 0 \\
& -84 & -11 l_{e s h_{i}} & 0 & 0 & -36 & 9 l_{e s h_{i}} & 0 \\
& & -2 l_{e s h_{i}}^{2} & 0 & 0 & -9 l_{e s h_{i}} & 2 l_{e s h_{i}}^{2} & 0 \\
& & & 0 & 0 & 0 & 0 & 0 \\
& & & & 0 & 0 & 0 & 0 \\
S & & & & & -84 & 11 l_{e s h_{i}} & 0 \\
Y & & & & & & -2 l_{e s h_{i}}^{2} & 0 \\
M & & & & & & & 0
\end{array}\right], \\
& \mathbf{M}_{e s h_{i}, 6}^{2}=\left[\begin{array}{cccccccc}
0 & 0 & 0 & 0 & 0 & 0 & 0 & 0 \\
& -40 & -5 l_{e s h_{i}} & 0 & 0 & -20 & 5 l_{e s h_{i}} & 0 \\
& & -l_{e s h_{i}}^{2} & 0 & 0 & -5 l_{e s h_{i}} & l_{e s h_{i}}^{2} & 0 \\
& & & 0 & 0 & 0 & 0 & 0 \\
& & & & 0 & 0 & 0 & 0 \\
S & & & & & -40 & 5 l_{e s h_{i}} & 0 \\
Y & & & & & & -l_{e s h_{i}}^{2} & 0 \\
M & & & & & & & 0
\end{array}\right] .
\end{aligned}
$$




$$
\begin{aligned}
& \mathbf{M}_{e s h_{i}, 7}=\frac{1}{30 l_{e s h_{i}}\left(1+\varphi_{e s h_{i}}^{x}\right)^{2}}\left(\mathbf{M}_{e s h_{i}, 7}^{0}+5 l_{e s h_{i}} \boldsymbol{\varphi}_{e s h_{i}}^{x} \mathbf{M}_{e s h_{i}, 7}^{1}+5 l_{e s h_{i}}^{2} \varphi_{e s h_{i}}^{x 2} \mathbf{M}_{e s h_{i}, 7}^{2}\right), \\
& \mathbf{M}_{e s h_{i}, 7}^{0}=\left[\begin{array}{cccccccc}
-36 & 0 & 0 & 3 l_{e s h_{i}} & 36 & 0 & 0 & 3 l_{e s h_{i}} \\
& 0 & 0 & 0 & 0 & 0 & 0 & 0 \\
& & 0 & 0 & 0 & 0 & 0 & 0 \\
& & & -4 l_{e s h_{i}}^{2} & -3 l_{e s h_{i}} & 0 & 0 & l_{e s h_{i}}^{2} \\
& & & & -36 & 0 & 0 & -3 l_{e s h_{i}} \\
S & & & & & 0 & 0 & 0 \\
Y & & & & & & 0 & 0 \\
M & & & & & & -4 l_{e s h_{i}}^{2}
\end{array}\right], \\
& \mathbf{M}_{e s h_{i}, 7}^{1}=\left[\begin{array}{cccccccc}
0 & 0 & 0 & -3 & 0 & 0 & 0 & -3 \\
& 0 & 0 & 0 & 0 & 0 & 0 & 0 \\
& & 0 & 0 & 0 & 0 & 0 & 0 \\
& & & -l_{e s h_{i}} & 3 & 0 & 0 & l_{e s h_{i}} \\
& & & & 0 & 0 & 0 & 3 \\
S & & & & & 0 & 0 & 0 \\
Y & & & & & & 0 & 0 \\
M & & & & & & & -l_{e s h_{i}}
\end{array}\right], \\
& \mathbf{M}_{e s h_{i}, 7}^{2}=\left[\begin{array}{cccccccc}
0 & 0 & 0 & 0 & 0 & 0 & 0 & 0 \\
& 0 & 0 & 0 & 0 & 0 & 0 & 0 \\
& & 0 & 0 & 0 & 0 & 0 & 0 \\
& & & -2 & 0 & 0 & 0 & -1 \\
& & & & 0 & 0 & 0 & 0 \\
S & & & & & 0 & 0 & 0 \\
Y & & & & & & 0 & 0 \\
M & & & & & & & -2
\end{array}\right] . \\
& \mathbf{M}_{e s h_{i}, 8}=\frac{1}{30 l_{e s h_{i}}\left(1+\varphi_{e s h_{i}}^{x}\right)^{2}}\left(\mathbf{M}_{e s h_{i}, 8}^{0}+5 \varphi_{e s h_{i}}^{z} \mathbf{M}_{e s h_{i}, 8}^{1}+\frac{5 \varphi_{e s h_{i}}^{22}}{2} \mathbf{M}_{e s h_{i}, 8}^{2}\right), \\
& \mathbf{M}_{e s h_{i}, 8}^{0}=\left[\begin{array}{cccccccc}
36 & 0 & 0 & -3 l_{e s h_{i}} & -36 & 0 & 0 & -3 l_{e s h_{i}} \\
& 36 v_{e s h_{i}}^{2} & 3 l_{e s h_{i}} v_{e s h_{i}}^{2} & 0 & 0 & -36 v_{e s h_{i}}^{2} & 3 l_{e s h_{i}} v_{e s h_{i}}^{2} & 0 \\
& & 4 l_{e s h_{i}}^{2} v_{e s h_{i}}^{2} & 0 & 0 & -3 l_{e s h_{h^{\prime}}} v_{e s h_{i}}^{2} & -l_{e s h_{i}}^{2} v_{e s h_{i}}^{2} & 0 \\
& & & 4 l_{e s h_{i}}^{2} & 3 l_{e s h_{i}} & 0 & 0 & -l_{e s h_{i}}^{2} \\
& & & & 36 & 0 & 0 & 3 l_{e s h_{i}} \\
S & & & & & 36 v_{e s h_{i}}^{2} & -3 l_{e s h_{i}} v_{e s h_{i}}^{2} & 0 \\
Y & & & & & & 4 l_{e s h_{i}}^{2} v_{e s h_{i}}^{2} & 0 \\
M & & & & & & & 4 l_{e s h_{i}}^{2}
\end{array}\right] \text {, } \\
& \mathbf{M}_{e s h_{i}, 8}^{1}=\left[\begin{array}{cccccccc}
12 \vartheta_{e s h_{i}} & 0 & 0 & 0 & -12 \vartheta_{e s h_{i}} & 0 & 0 & 0 \\
& 12 v_{e s h_{i}}^{2} & 0 & 0 & 0 & -12 v_{e s h_{i}}^{2} & 0 & 0 \\
& & l_{e s h_{i}}^{2} v_{e s h_{i}}^{2} & 0 & 0 & 0 & -l_{e s h_{i}}^{2} v_{e s h_{i}}^{2} & 0 \\
& & & l_{e s h_{i}}^{2} \vartheta_{e s h_{i}} & 0 & 0 & 0 & -l_{e s h_{i}}^{2} \vartheta_{e s h_{i}} \\
& & & & 12 \vartheta_{e s h_{i}} & 0 & 0 & 0 \\
S & & & & & 12 v_{e s h_{i}}^{2} & 0 & 0 \\
Y & & & & & & l_{e s h_{i}}^{2} v_{e s h_{i}}^{2} & 0 \\
M & & & & & & & l_{e s h_{i}}^{2} \vartheta_{e s h_{i}}
\end{array}\right] \text {, } \\
& \mathbf{M}_{e s h_{i}, 8}^{2}=\left[\begin{array}{cccccccc}
12 \vartheta_{e s h_{i}}^{2} & 0 & 0 & 0 & -12 \vartheta_{e s h_{i}}^{2} & 0 & 0 & 0 \\
& 12 v_{e s h_{i}}^{2} & 0 & 0 & 0 & -12 v_{e s h_{i}}^{2} & 0 & 0 \\
& & l_{e s h_{i}}^{2} v_{e s h_{i}}^{2} & 0 & 0 & 0 & -l_{e s h_{i}}^{2} v_{e s h_{i}}^{2} & 0 \\
& & & l_{e s h_{i}}^{2} \vartheta_{e s h_{i}}^{2} & 0 & 0 & 0 & -l_{e s h_{i}}^{2} \vartheta_{e s h_{i}}^{2} \\
& & & & 12 \vartheta_{e s h_{i}}^{2} & 0 & 0 & 0 \\
S & & & & & 12 v_{e s h_{i}}^{2} & 0 & 0 \\
Y & & & & & & l_{e s h_{i}}^{2} v_{e s h_{i}}^{2} & 0 \\
M & & & & & & & l_{e s h_{i}}^{2} \vartheta_{e s h_{i}}^{2}
\end{array}\right] .
\end{aligned}
$$




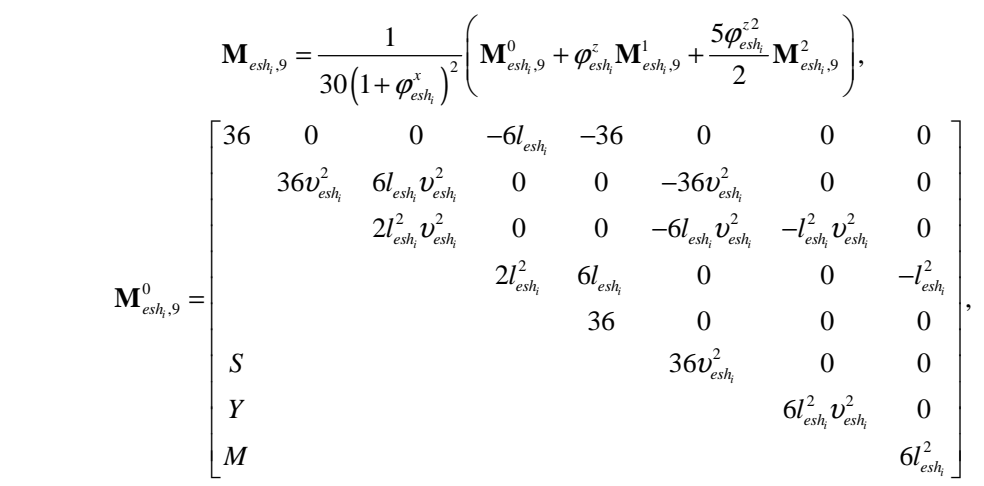

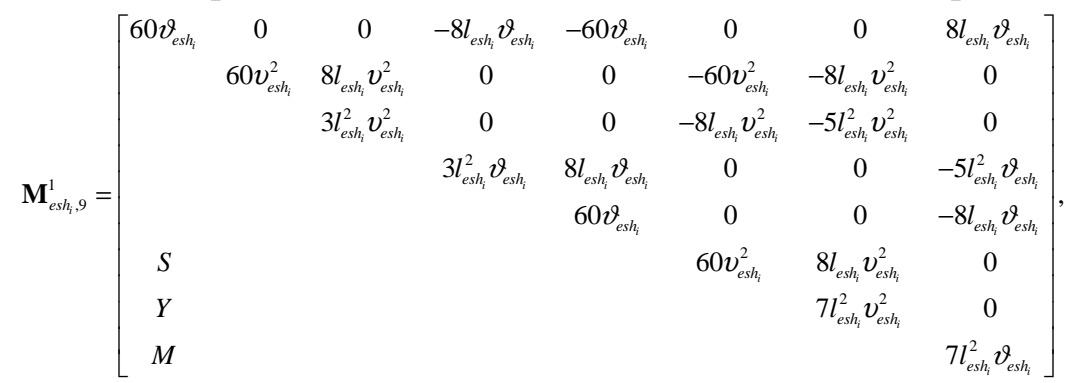

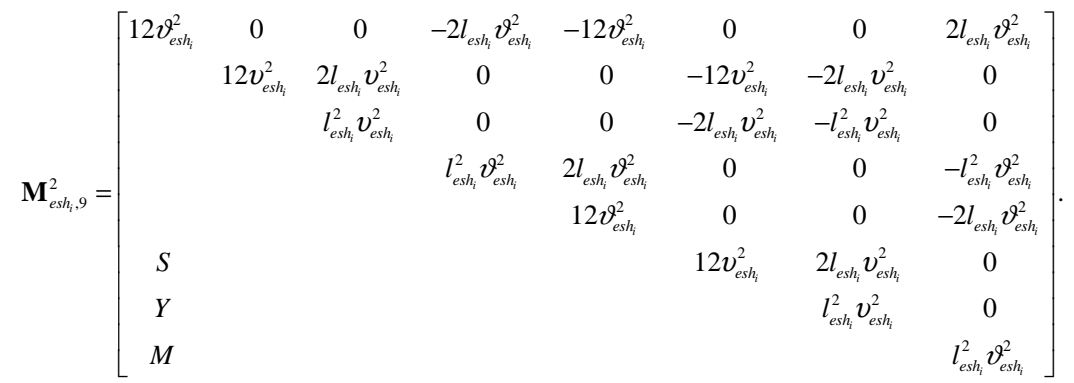

$$
\begin{aligned}
& \mathbf{M}_{e s s_{h}, 10}=\frac{l_{e s h_{h}}}{210\left(1+\varphi_{e s h_{h}}^{x}\right.}\left(\mathbf{M}_{e s h_{1}, 10}^{0}+\frac{7 \varphi_{e s h_{h}}^{2}}{2} \mathbf{M}_{e s h_{h}, 10}^{1}+\frac{7 \varphi_{e s_{h}}^{22}}{2} \mathbf{M}_{e s h_{h}, 10}^{2}\right),
\end{aligned}
$$

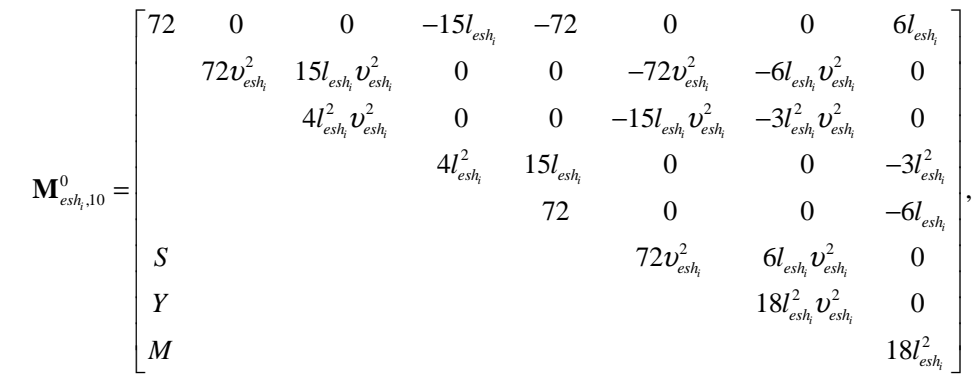

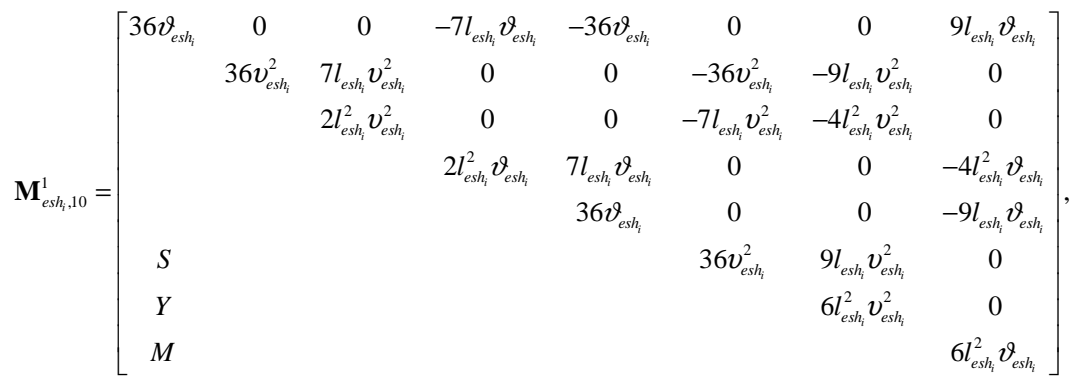

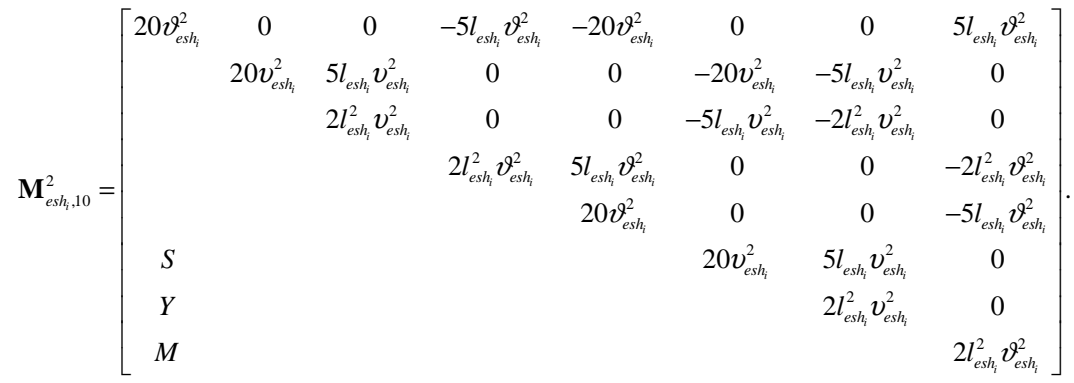




$$
\begin{aligned}
& \mathbf{V}_{e s h_{i}, 1}=\frac{l_{e s h_{i}}}{12}\left\{\begin{array}{c}
6 \\
0 \\
0 \\
-l_{e s h_{i}} \\
6 \\
0 \\
0 \\
l_{e s h_{i}}
\end{array}\right\} ; \quad \mathbf{V}_{e s h_{i}, 2}=\frac{l_{e s h_{i}}}{12}\left\{\begin{array}{c}
0 \\
6 \\
l_{e s h_{i}} \\
0 \\
0 \\
6 \\
-l_{e s h_{i}} \\
0
\end{array}\right\} ; \quad \mathbf{V}_{e s h_{i}, 3}=\frac{1}{2\left(1+\varphi_{e s h_{i}}^{x}\right)}\left\{\begin{array}{c}
2 \\
0 \\
0 \\
l_{e s h_{i}} \varphi_{e s h_{i}}^{x} \\
-2 \\
0 \\
0 \\
l_{e s h_{i}} \varphi_{e_{s h_{i}}^{x}}^{x}
\end{array}\right\} .
\end{aligned}
$$

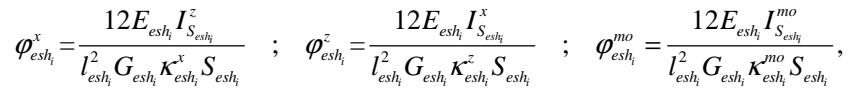

$$
\begin{aligned}
& v_{e s h_{i}}=\frac{1+\varphi_{e s h_{i}}^{x}}{1+\varphi_{e s h_{i}}^{z}} ; \quad \vartheta_{e s h_{i}}=\frac{\varphi_{e s h_{i}}^{x}}{\varphi_{e s h_{i}}^{z}} .
\end{aligned}
$$

The elementary vectors for the mass unbalance are expressed by

$$
\begin{aligned}
& \mathbf{V}_{e m u}^{c}=m_{m u} r_{m u}\left\{\begin{array}{c}
\sin \eta_{m u} \\
\cos \eta_{m u} \\
0 \\
0
\end{array}\right\} ; \quad \mathbf{V}_{e m u}^{s}=m_{m u} r_{m u}\left\{\begin{array}{c}
\cos \eta_{m u} \\
-\sin \eta_{m u} \\
0 \\
0
\end{array}\right\} ; \quad \mathbf{V}_{e m u, b}^{\omega^{r^{2}, c}, c}=m_{m u} r_{m u}\left\{\begin{array}{c}
0 \\
\cos \eta_{m u} \\
0 \\
0
\end{array}\right\} ; \quad \mathbf{V}_{e m u, b}^{v^{2 !}, s}=m_{m u} r_{m u}\left\{\begin{array}{c}
0 \\
-\sin \eta_{m u} \\
0 \\
0
\end{array}\right\} \text {, } \\
& \mathbf{V}_{e m u, b}^{\Omega \omega^{y}, c}=2 m_{m u} r_{m u}\left\{\begin{array}{c}
\sin \eta_{m u} \\
\cos \eta_{m u} \\
0 \\
0
\end{array}\right\} ; \quad \mathbf{V}_{e m u, b}^{\Omega \omega^{y}, s}=2 m_{m u} r_{m u}\left(\begin{array}{c}
\cos \eta_{m u} \\
-\sin \eta_{m u} \\
0 \\
0
\end{array}\right\} ; \quad \mathbf{V}_{e m u, b}^{\omega^{y 2}, c}=m_{m u} r_{m u}\left(\begin{array}{c}
\sin \eta_{m u} \\
\cos \eta_{m u} \\
0 \\
0
\end{array}\right\} ; \quad \mathbf{V}_{e m u, b}^{\omega^{y 2}, s}=m_{m u} r_{m u}\left\{\begin{array}{c}
\cos \eta_{m u} \\
-\sin \eta_{m u} \\
0 \\
0
\end{array}\right\} \text {. }
\end{aligned}
$$

